\title{
Spectral action on noncommutative torus
}

\author{
Driss Essouabri, Bruno Iochum, Cyril Levy, and Andrzej Sitarz*
}

Dedicated to Alain Connes on the occasion of his 60th birthday

\begin{abstract}
The spectral action on the noncommutative torus is obtained using a ChamseddineConnes formula via computations of zeta functions. The importance of a Diophantine condition is outlined. Several results on holomorphic continuation of series of holomorphic functions are obtained in this context.
\end{abstract}

PACS numbers. 11.10.Nx, 02.30.Sa, 11.15.Kc.

Mathematics Subject Classification (2000). 46H35, 46L52, 58B34.

Keywords. Spectral action, noncommutative torus, zeta function, holomorphic continuation, Diophantine number.

\section{Contents}

1 Introduction . . . . . . . . . . . . . . . . . . 53

2 Residues of series and integral, holomorphic continuation, etc. . . . . . . . . . 55

3 Noncommutative integration on a simple spectral triple . . . . . . . . . . . . . 74

4 Residues of $\zeta_{D_{A}}$ for a spectral triple with simple dimension spectrum . . . . . . . . 78

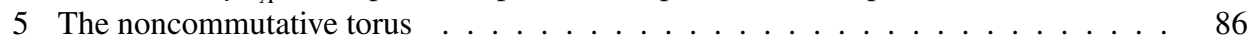

6 The spectral action . . . . . . . . . . . . . . . . . . . . . . 97

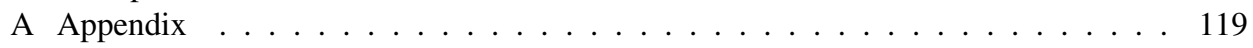

\section{Introduction}

The spectral action introduced by Chamseddine-Connes plays an important role [3] in noncommutative geometry. More precisely, given a spectral triple $(\mathcal{A}, \mathcal{H}, \mathcal{D})$ where $\mathcal{A}$ is an algebra acting on the Hilbert space $\mathcal{H}$ and $\mathcal{D}$ is a Dirac-like operator (see [7], [23]), Chamseddine and Connes proposed a physical action depending only on the spectrum of the covariant Dirac operator

$$
\mathcal{D}_{A}:=\mathcal{D}+A+\epsilon J A J^{-1},
$$

\footnotetext{
*Partially supported by MNII Grant 115/E-343/SPB/6.PR UE/DIE 50/2005-2008.
} 
where $A$ is a 1 -form represented on $\mathcal{H}$, so has the decomposition

$$
A=\sum_{i} a_{i}\left[\mathcal{D}, b_{i}\right]
$$

with $a_{i}, b_{i} \in \mathcal{A}, J$ is a real structure on the triple corresponding to charge conjugation and $\epsilon \in\{1,-1\}$ depends on the dimension of this triple and comes from the commutation relation

$$
J \mathcal{D}=\epsilon \mathcal{D} J
$$

This action is defined by

$$
\mathcal{S}\left(\mathcal{D}_{A}, \Phi, \Lambda\right):=\operatorname{Tr}\left(\Phi\left(\mathcal{D}_{A} / \Lambda\right)\right)
$$

where $\Phi$ is any even positive cut-off function which could be replaced by a step function up to some mathematical difficulties investigated in [16]. This means that $\mathcal{S}$ counts the spectral values of $\left|\mathcal{D}_{A}\right|$ that are less than the mass scale $\Lambda$ (note that the resolvent of $\mathcal{D}_{A}$ is compact since, by assumption, the same is true for $\mathcal{D}$; see Lemma 3.1 below).

In [18], the spectral action on NC-tori has been computed only for operators of the form $\mathcal{D}+A$ and for $\mathcal{D}_{A}$ in [20]. It appears that the implementation of the real structure via $J$ does change the spectral action up to a coefficient when the torus has dimension 4. Here we prove that this can be also directly obtained from the Chamseddine-Connes analysis in [11] that we follow quite closely. Actually,

$$
\mathcal{S}\left(\mathcal{D}_{A}, \Phi, \Lambda\right)=\sum_{0<k \in \mathrm{Sd}^{+}} \Phi_{k} \Lambda^{k} f\left|D_{A}\right|^{-k}+\Phi(0) \zeta_{D_{A}}(0)+\mathcal{O}\left(\Lambda^{-1}\right)
$$

where $D_{A}=\mathcal{D}_{A}+P_{A}, P_{A}$ is the projection on $\operatorname{Ker} \mathcal{D}_{A}, \Phi_{k}=\frac{1}{2} \int_{0}^{\infty} \Phi(t) t^{k / 2-1} d t$ and $\mathrm{Sd}^{+}$is the strictly positive part of the dimension spectrum of $(\mathcal{A}, \mathcal{H}, \mathcal{D})$. As we will see, $\mathrm{Sd}^{+}=\{1,2, \ldots, n\}$ and $f\left|D_{A}\right|^{-n}=f|D|^{-n}$. Moreover, the coefficient $\zeta_{D_{A}}(0)$ related to the constant term in (1.5) can be computed from the unperturbed spectral action since it has been proved in [11] (with an invertible Dirac operator and a 1 -form $A$ such that $\mathcal{D}+A$ is also invertible) that

$$
\zeta_{\mathcal{D}+A}(0)-\zeta_{\mathcal{D}}(0)=\sum_{q=1}^{n} \frac{(-1)^{q}}{q} f\left(A \mathcal{D}^{-1}\right)^{q}
$$

using $\zeta_{X}(s)=\operatorname{Tr}\left(|X|^{-s}\right)$. We will see how this formula can be extended to the case of a noninvertible Dirac operator and noninvertible perturbation of the form $\mathcal{D}+\tilde{A}$ with $\tilde{A}:=A+\varepsilon J A J^{-1}$.

All these results on spectral action are quite important in physics, especially in quantum field theory and particle physics, where one adds to the effective action some 
counterterms explicitly given by (1.6); see for instance [2], [3], [4], [11], [17], [18], [20], [22], [28], [34], [36], [37], [38].

Since the computation of zeta functions is crucial in the following, we investigate in Section 2 residues of series and integrals. This section contains results of independent interest on the holomorphy of series of holomorphic functions. In particular, the necessity of a Diophantine constraint is naturally emphasized.

In Section 3 we revisit the notions of pseudodifferential operators and their associated zeta functions and of dimension spectrum. The reality operator $J$ is incorporated, and we pay a particular attention to kernels of operators that can play a role in the constant term of (1.5). This section concerns general spectral triples with simple dimension spectrum.

Section 4 is devoted to the example of the noncommutative torus. It is shown that it has a vanishing tadpole.

In Section 5 all previous technical points are then widely used for the computation of terms in (1.5) or (1.6).

Finally, the spectral action (1.6) is obtained in Section 6, and we conjecture that the noncommutative spectral action of $\mathcal{D}_{A}$ has terms proportional to the spectral action of $\mathcal{D}+A$ on the commutative torus.

\section{Residues of series and integral, holomorphic continuation, etc.}

Notations. In the following the prime in $\sum^{\prime}$ means that we omit terms with division by zero in the summand. $B^{n}$ (resp. $S^{n-1}$ ) is the closed ball (resp. the sphere) of $\mathbb{R}^{n}$ with center 0 and radius 1 . The Lebesgue measure on $S^{n-1}$ will be noted by $d S$.

For any $x=\left(x_{1}, \ldots, x_{n}\right) \in \mathbb{R}^{n}$ we denote by $|x|=\sqrt{x_{1}^{2}+\cdots+x_{n}^{2}}$ the Euclidean norm and $|x|_{1}:=\left|x_{1}\right|+\cdots+\left|x_{n}\right|$.

$\mathbb{N}=\{1,2, \ldots\}$ is the set of positive integers and $\mathbb{N}_{0}=\mathbb{N} \cup\{0\}$ the set of non-negative integers.

By $f(x, y) \ll y g(x)$ uniformly in $x$, we mean that, for some $a(y)>0$, $|f(x, y)| \leq a(y)|g(x)|$ for all $x$ and $y$.

2.1. Residues of series and integral. In order to be able to compute later the residues of certain series, we now prove the following result.

Theorem 2.1. Let $P(X)=\sum_{j=0}^{d} P_{j}(X) \in \mathbb{C}\left[X_{1}, \ldots, X_{n}\right]$ be a polynomial function where $P_{j}$ is the homogeneous part of $P$ of degree $j$. The function

$$
\zeta^{P}(s):=\sum_{k \in \mathbb{Z}^{n}}^{\prime} \frac{P(k)}{|k|^{s}}, \quad s \in \mathbb{C},
$$

has a meromorphic continuation to the whole complex plane $\mathbb{C}$. 
Also $\zeta^{P}(s)$ is not entire if and only if $\mathcal{P}_{P}:=\left\{j \mid \int_{u \in S^{n-1}} P_{j}(u) d S(u) \neq 0\right\} \neq \varnothing$. In that case $\zeta^{P}$ has only simple poles at the points $j+n, j \in \mathcal{P}_{P}$, with

$$
\operatorname{Res}_{s=j+n} \zeta^{P}(s)=\int_{u \in S^{n-1}} P_{j}(u) d S(u) .
$$

The proof of this theorem is based on the following lemmas.

Lemma 2.2. For any polynomial $P \in \mathbb{C}\left[X_{1}, \ldots, X_{n}\right]$ of total degree $\delta(P):=$ $\sum_{i=1}^{n} \operatorname{deg}_{X_{i}} P$ and any $\alpha \in \mathbb{N}_{0}^{n}$, we have

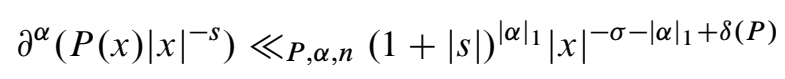

uniformly in $x \in \mathbb{R}^{n},|x| \geq 1$, where $\sigma=\operatorname{Re}(s)$.

Proof. By linearity, we may assume without loss of generality that $P(X)=X^{\gamma}$ is a monomial. It is easy to prove (for example by induction on $|\alpha|_{1}$ ) that for all $\alpha \in \mathbb{N}_{0}^{n}$ and $x \in \mathbb{R}^{n} \backslash\{0\}$ :

$$
\partial^{\alpha}\left(|x|^{-s}\right)=\alpha ! \sum_{\substack{\beta, \mu \in \mathbb{N}_{0}^{n} \\
\beta+2 \mu=\alpha}}\left(\begin{array}{c}
-s / 2 \\
|\beta|_{1}+|\mu|_{1}
\end{array}\right) \frac{\left(|\beta|_{1}+|\mu|_{1}\right) !}{\beta ! \mu !} \frac{x^{\beta}}{|x|^{\sigma+2\left(|\beta|_{1}+|\mu|_{1}\right)}} .
$$

It follows that for all $\alpha \in \mathbb{N}_{0}^{n}$, we have uniformly in $x \in \mathbb{R}^{n},|x| \geq 1$,

$$
\partial^{\alpha}\left(|x|^{-s}\right) \ll_{\alpha, n}(1+|s|)^{|\alpha|_{1}}|x|^{-\sigma-|\alpha|_{1}} .
$$

By the Leibniz formula and (2.1), we have uniformly in $x \in \mathbb{R}^{n},|x| \geq 1$,

$$
\begin{aligned}
\partial^{\alpha}\left(x^{\gamma}|x|^{-s}\right) & =\sum_{\beta \leq \alpha}\left(\begin{array}{c}
\alpha \\
\beta
\end{array}\right) \partial^{\beta}\left(x^{\gamma}\right) \partial^{\alpha-\beta}\left(|x|^{-s}\right) \\
& \ll \gamma, \alpha, n \sum_{\beta \leq \alpha ; \beta \leq \gamma} x^{\gamma-\beta}(1+|s|)^{|\alpha|_{1}-|\beta|_{1}}|x|^{-\sigma-|\alpha|_{1}+|\beta|_{1}} \\
& \ll_{\gamma, \alpha, n}(1+|s|)^{|\alpha|_{1}}|x|^{-\sigma-|\alpha|_{1}+|\gamma|_{1}} .
\end{aligned}
$$

Lemma 2.3. Let $P \in \mathbb{C}\left[X_{1}, \ldots, X_{n}\right]$ be a polynomial of degree $d$. Then the difference

$$
\Delta_{P}(s):=\sum_{k \in \mathbb{Z}^{n}}^{\prime} \frac{P(k)}{|k|^{s}}-\int_{\mathbb{R}^{n} \backslash B^{n}} \frac{P(x)}{|x|^{s}} d x,
$$

which is defined for $\operatorname{Re}(s)>d+n$, extends holomorphically to the whole complex plane $\mathbb{C}$. 
Proof. We fix in the sequel a function $\psi \in C^{\infty}\left(\mathbb{R}^{n}, \mathbb{R}\right)$ such that for all $x \in \mathbb{R}^{n}$ we have

$$
0 \leq \psi(x) \leq 1, \quad \psi(x)=1 \text { if }|x| \geq 1 \quad \text { and } \quad \psi(x)=0 \text { if }|x| \leq 1 / 2 .
$$

The function $f(x, s):=\psi(x) P(x)|x|^{-s}, x \in \mathbb{R}^{n}$ and $s \in \mathbb{C}$, is in $\mathcal{C}^{\infty}\left(\mathbb{R}^{n} \times \mathbb{C}\right)$ and depends holomorphically on $s$.

Lemma 2.2 above shows that $f$ is a "gauged symbol" in the terminology of [24], p. 4. Thus [24], Theorem 2.1, implies that $\Delta_{P}(s)$ extends holomorphically to the whole complex plane $\mathbb{C}$. However, for completeness, we will give here a short proof of Lemma 2.3:

It follows from the classical Euler-Maclaurin formula that for any function $h: \mathbb{R} \rightarrow \mathbb{C}$ of class $\mathrm{C}^{N+1}$ satisfying $\lim _{|t| \rightarrow+\infty} h^{(k)}(t)=0$ and $\int_{\mathbb{R}}\left|h^{(k)}(t)\right| d t<$ $+\infty$ for any $k=0, \ldots, N+1$, we have

$$
\sum_{k \in \mathbb{Z}} h(k)=\int_{\mathbb{R}} h(t)+\frac{(-1)^{N}}{(N+1) !} \int_{\mathbb{R}} B_{N+1}(t) h^{(N+1)}(t) d t,
$$

where $B_{N+1}$ is the Bernoulli function of order $N+1$ (it is a bounded periodic function).

Fix $m^{\prime} \in \mathbb{Z}^{n-1}$ and $s \in \mathbb{C}$. Applying this to the function

$$
h(t):=\psi\left(m^{\prime}, t\right) P\left(m^{\prime}, t\right)\left|\left(m^{\prime}, t\right)\right|^{-s}
$$

(we use Lemma 2.2 to verify the hypothesis), we obtain that for any $N \in \mathbb{N}_{0}$,

$$
\begin{aligned}
\sum_{m_{n} \in \mathbb{Z}} \psi\left(m^{\prime}, m_{n}\right) P\left(m^{\prime}, m_{n}\right)\left|\left(m^{\prime}, m_{n}\right)\right|^{-s} \\
\quad=\int_{\mathbb{R}} \psi\left(m^{\prime}, t\right) P\left(m^{\prime}, t\right)\left|\left(m^{\prime}, t\right)\right|^{-s} d t+\mathcal{R}_{N}\left(m^{\prime} ; s\right)
\end{aligned}
$$

where $\mathcal{R}_{N}\left(m^{\prime} ; s\right):=\frac{(-1)^{N}}{(N+1) !} \int_{\mathbb{R}} B_{N+1}(t) \frac{\partial^{N+1}}{\partial x_{n}{ }^{N+1}}\left(\psi\left(m^{\prime}, t\right) P\left(m^{\prime}, t\right)\left|\left(m^{\prime}, t\right)\right|^{-s}\right) d t$.

By Lemma 2.2,

$$
\begin{gathered}
\int_{\mathbb{R}}\left|B_{N+1}(t) \frac{\partial^{N+1}}{\partial x_{n}{ }^{N+1}}\left(\psi\left(m^{\prime}, t\right) P\left(m^{\prime}, t\right)\left|\left(m^{\prime}, t\right)\right|^{-s}\right)\right| d t \\
\ll P, n, N(1+|s|)^{N+1}\left(\left|m^{\prime}\right|+1\right)^{-\sigma-N+\delta(P)} .
\end{gathered}
$$

Thus $\sum_{m^{\prime} \in \mathbb{Z}^{n-1}} \mathcal{R}_{N}\left(m^{\prime} ; s\right)$ converges absolutely and defines a holomorphic function in the half plane $\{\sigma=\operatorname{Re}(s)>\delta(P)+n-N\}$. 
Since $N$ is an arbitrary integer, by letting $N \rightarrow \infty$ and using (2.2) above, we conclude that

$$
\begin{aligned}
s \mapsto \sum_{\left(m^{\prime}, m_{n}\right) \in \mathbb{Z}^{n-1} \times \mathbb{Z}} \psi\left(m^{\prime}, m_{n}\right) P\left(m^{\prime}, m_{n}\right)\left|\left(m^{\prime}, m_{n}\right)\right|^{-s} & \\
& -\sum_{m^{\prime} \in \mathbb{Z}^{n-1}} \int_{\mathbb{R}} \psi\left(m^{\prime}, t\right) P\left(m^{\prime}, t\right)\left|\left(m^{\prime}, t\right)\right|^{-s} d t
\end{aligned}
$$

has a holomorphic continuation to the whole complex plane $\mathbb{C}$.

After $n$ iterations, we obtain that

$$
s \mapsto \sum_{m \in \mathbb{Z}^{n}} \psi(m) P(m)|m|^{-s}-\int_{\mathbb{R}^{n}} \psi(x) P(x)|x|^{-s} d x
$$

has a holomorphic continuation to the whole of $\mathbb{C}$.

To finish the proof of Lemma 2.3, it is enough to notice that

- $\psi(0)=0$ and $\psi(m)=1$ for all $m \in \mathbb{Z}^{n} \backslash\{0\}$;

- $s \mapsto \int_{B^{n}} \psi(x) P(x)|x|^{-s} d x=\int_{\left\{x \in \mathbb{R}^{n}|1 / 2 \leq| x \mid \leq 1\right\}} \psi(x) P(x)|x|^{-s} d x$ is a holomorphic function in $\mathbb{C}$.

Proof of Theorem 2.1. Using the polar decomposition of the volume form $d x=$ $\rho^{n-1} d \rho d S$ in $\mathbb{R}^{n}$, we obtain for $\operatorname{Re}(s)>d+n$ that

$$
\begin{aligned}
\int_{\mathbb{R}^{n} \backslash B^{n}} \frac{P_{j}(x)}{|x|^{s}} d x & =\int_{1}^{\infty} \frac{\rho^{j+n-1}}{\rho^{s}} \int_{S^{n-1}} P_{j}(u) d S(u) \\
& =\frac{1}{j+n-s} \int_{S^{n-1}} P_{j}(u) d S(u) .
\end{aligned}
$$

Lemma 2.3 now gives the result.

2.2. Holomorphy of certain series. Before stating the main result of this section, we give first in the following some preliminaries from Diophantine approximation theory.

Definition 2.4. (i) Let $\delta>0$. A vector $a \in \mathbb{R}^{n}$ is said to be $\delta$-diophantine if there exists $c>0$ such that $|q \cdot a-m| \geq c|q|^{-\delta}$ for all $q \in \mathbb{Z}^{n} \backslash\{0\}$ and for all $m \in \mathbb{Z}$.

We denote $\mathcal{B} \mathcal{V}(\delta)$ the set of $\delta$-diophantine vectors and $\mathcal{B} \mathcal{V}:=\bigcup_{\delta>0} \mathcal{B} \mathcal{V}(\delta)$ the set of diophantine vectors.

(ii) A matrix $\Theta \in \mathcal{M}_{n}(\mathbb{R})$ (real $n \times n$ matrices) is said to be diophantine if there exists $u \in \mathbb{Z}^{n}$ such that ${ }^{t} \Theta(u)$ is a diophantine vector of $\mathbb{R}^{n}$. 
Remark 2.5. A classical result from Diophantine approximation asserts that for all $\delta>n$, the Lebesgue measure of $\mathbb{R}^{n} \backslash \mathcal{B} \mathcal{V}(\delta)$ is zero (i.e., almost any element of $\mathbb{R}^{n}$ is $\delta$-diophantine).

Let $\Theta \in \mathcal{M}_{n}(\mathbb{R})$. If its row of index $i$ is a diophantine vector of $\mathbb{R}^{n}$ (i.e., if $\left.L_{i} \in \mathcal{B V}\right)$ then ${ }^{t} \Theta\left(e_{i}\right) \in \mathcal{B V}$ and thus $\Theta$ is a diophantine matrix. It follows that almost any matrix of $\mathcal{M}_{n}(\mathbb{R}) \approx \mathbb{R}^{n^{2}}$ is diophantine.

The goal of this section is to show the following result.

Theorem 2.6. Let $P \in \mathbb{C}\left[X_{1}, \ldots, X_{n}\right]$ be a homogeneous polynomial of degree $d$ and let $b$ be in $\mathcal{S}\left(\mathbb{Z}^{n} \times \cdots \times \mathbb{Z}^{n}\right)$ ( $q$ times, $\left.q \in \mathbb{N}\right)$. Then the following holds:

(i) Let $a \in \mathbb{R}^{n}$. We define $f_{a}(s):=\sum_{k \in \mathbb{Z}^{n}}^{\prime} \frac{P(k)}{|k|^{s}} e^{2 \pi i k \cdot a}$.

1. If $a \in \mathbb{Z}^{n}$, then $f_{a}$ has a meromorphic continuation to the whole complex plane $\mathbb{C}$.

Moreover if $S$ is the unit sphere and $d S$ its Lebesgue measure, then $f_{a}$ is not entire if and only if $\int_{u \in S^{n-1}} P(u) d S(u) \neq 0$. In that case, $f_{a}$ has only a simple pole at the point $d+n$ with $\operatorname{Res}_{s=d+n} f_{a}(s)=\int_{u \in S^{n-1}} P(u) d S(u)$.

2. If $a \in \mathbb{R}^{n} \backslash \mathbb{Z}^{n}$, then $f_{a}(s)$ extends holomorphically to the whole complex plane $\mathbb{C}$.

(ii) Suppose that $\Theta \in \mathcal{M}_{n}(\mathbb{R})$ is diophantine. For any $\left(\varepsilon_{i}\right)_{i} \in\{-1,0,1\}^{q}$, the function

$$
g(s):=\sum_{l \in\left(\mathbb{Z}^{n}\right)^{q}} b(l) f_{\Theta \sum_{i} \varepsilon_{i} l_{i}}(s)
$$

extends meromorphically to the whole complex plane $\mathbb{C}$ with only one possible pole on $s=d+n$.

Moreover, if we set $Z:=\left\{l \in\left(\mathbb{Z}^{n}\right)^{q} \mid \sum_{i=1}^{q} \varepsilon_{i} l_{i}=0\right\}$ and $V:=\sum_{l \in Z} b(l)$, then:

1. If $V \int_{S^{n-1}} P(u) d S(u) \neq 0$, then $s=d+n$ is a simple pole of $g(s)$ and

$$
\operatorname{Res}_{s=d+n} g(s)=V \int_{u \in S^{n-1}} P(u) d S(u) .
$$

2. If $V \int_{S^{n-1}} P(u) d S(u)=0$, then $g(s)$ extends holomorphically to the whole complex plane $\mathbb{C}$.

(iii) Suppose that $\Theta \in \mathcal{M}_{n}(\mathbb{R})$ is diophantine. For any $\left(\varepsilon_{i}\right)_{i} \in\{-1,0,1\}^{q}$, the function

$$
g_{0}(s):=\sum_{l \in\left(\mathbb{Z}^{n}\right)^{q} \backslash z} b(l) f_{\Theta \sum_{i=1}^{q} \varepsilon_{i} l_{i}}(s)
$$

where $Z:=\left\{l \in\left(\mathbb{Z}^{n}\right)^{q} \mid \sum_{i=1}^{q} \varepsilon_{i} l_{i}=0\right\}$ extends holomorphically to the whole complex plane $\mathbb{C}$. 
Proof of Theorem 2.6. First we remark that if $a \in \mathbb{Z}^{n}$ then $f_{a}(s)=\sum_{k \in \mathbb{Z}^{n}}^{\prime} \frac{P(k)}{|k|^{s}}$, so (i.1) follows from Theorem 2.1.

Further, $g(s):=\sum_{l \in\left(\mathbb{Z}^{n}\right)^{q} \backslash z} b(l) f_{\Theta \sum_{i} \varepsilon_{i} l_{i}}(s)+\left(\sum_{l \in Z} b(l)\right) \sum_{k \in \mathbb{Z}^{n}}^{\prime} \frac{P(k)}{|k|^{s}}$, so (ii) follows easily from (iii) and Theorem 2.1 .

Therefore, to complete the proof it remains to show (i.2) and (iii).

The direct proof of (i.2) is easy, but it is not sufficient to deduce (iii), the proof of which is more delicate and requires a more precise (i.e., more effective) version of (i.2). The next lemma gives such crucial version, but before let us introduce some notations. Let

$$
\mathcal{F}:=\left\{\frac{P(X)}{\left(X_{1}^{2}+\cdots+X_{n}^{2}+1\right)^{r / 2}} \mid P(X) \in \mathbb{C}\left[X_{1}, \ldots, X_{n}\right] \text { and } r \in \mathbb{N}_{0}\right\},
$$

and put $g=\operatorname{deg}(G)=\operatorname{deg}(P)-r \in \mathbb{Z}$, where $G=\frac{P(X)}{\left(X_{1}^{2}+\cdots+X_{n}^{2}+1\right)^{r / 2}} \in \mathcal{F}$. Moreover, we set $\operatorname{deg}(0)=-\infty$.

Lemma 2.7. Let $a \in \mathbb{R}^{n}$. We assume that $d(a \cdot u, \mathbb{Z}):=\inf _{m \in \mathbb{Z}}|a \cdot u-m|>0$ for some $u \in \mathbb{Z}^{n}$. For all $G \in \mathcal{F}$, we define formally

$$
F_{0}(G ; a ; s):=\sum_{k \in \mathbb{Z}^{n}}^{\prime} \frac{G(k)}{|k|^{s}} e^{2 \pi i k \cdot a} \text { and } F_{1}(G ; a ; s):=\sum_{k \in \mathbb{Z}^{n}} \frac{G(k)}{\left(|k|^{2}+1\right)^{s / 2}} e^{2 \pi i k \cdot a} .
$$

Then for all $N \in \mathbb{N}$, all $G \in \mathcal{F}$ and all $i \in\{0,1\}$, there exist positive constants $C_{i}:=C_{i}(G, N, u), B_{i}:=B_{i}(G, N, u)$ and $A_{i}:=A_{i}(G, N, u)$ such that $s \mapsto$ $F_{i}(G ; \alpha ; s)$ extends holomorphically to the half plane $\{\operatorname{Re}(s)>-N\}$ and satisfies

$$
F_{i}(G ; a ; s) \leq C_{i}(1+|s|)^{B_{i}}(d(a \cdot u, \mathbb{Z}))^{-A_{i}} .
$$

Remark 2.8. The important point here is that we obtain an explicit bound of $F_{i}(G ; \alpha ; s)$ in $\{\operatorname{Re}(s)>-N\}$ which depends on the vector $a$ only through $d(a \cdot u, \mathbb{Z})$, so depends on $u$ and indirectly on $a$ (in the sequel, $a$ will vary). In particular, the constants $C_{i}:=C_{i}(G, N, u), B_{i}=B_{i}(G, N)$ and $A_{i}:=A_{i}(G, N)$ do not depend on the vector $a$ but only on $u$. This is crucial for the proof of (ii) and (iii) in Theorem 2.6.

2.2.1. Proof of Lemma 2.7 for $\boldsymbol{i}=\mathbf{1}$. Let $N \in \mathbb{N}_{0}$ be a fixed integer and set $g_{0}:=n+N+1$. We will prove Lemma 2.7 by induction on $g=\operatorname{deg}(G) \in \mathbb{Z}$. More precisely, in order to prove the case $i=1$, it suffices to show that Lemma 2.7 is true for all $G \in \mathcal{F}$ with $\operatorname{deg}(G) \leq-g_{0}$.

Let $g \in \mathbb{Z}$ with $g \geq-g_{0}+1$. If Lemma 2.7 is true for all $G \in \mathcal{F}$ such that $\operatorname{deg}(G) \leq g-1$, then it is also true for all $G \in \mathcal{F}$ satisfying $\operatorname{deg}(G)=g$.

Step 1: Checking Lemma 2.7 for $\operatorname{deg}(G) \leq-g_{0}:=-(n+N+1)$. 
Let $G(X)=\frac{P(X)}{\left(X_{1}^{2}+\cdots+X_{n}^{2}+1\right)^{r / 2}} \in \mathcal{F}$ with $\operatorname{deg}(G) \leq-g_{0}$. It is easy to see that we have uniformly in $s=\sigma+i \tau \in \mathbb{C}$ and in $k \in \mathbb{Z}^{n}$ :

$$
\begin{aligned}
\frac{\left|G(k) e^{2 \pi i k \cdot a}\right|}{\left(|k|^{2}+1\right)^{\sigma / 2}} & =\frac{|P(k)|}{\left(|k|^{2}+1\right)^{(r+\sigma) / 2}} \\
& \ll_{G} \frac{1}{\left(|k|^{2}+1\right)^{(r+\sigma-\operatorname{deg}(P)) / 2}} \\
& \ll_{G} \frac{1}{\left(|k|^{2}+1\right)^{(\sigma-\operatorname{deg}(G)) / 2}} \\
& \ll_{G} \frac{1}{\left(|k|^{2}+1\right)^{\left(\sigma+g_{0}\right) / 2}} .
\end{aligned}
$$

It follows that $F_{1}(G ; a ; s)=\sum_{k \in \mathbb{Z}^{n}} \frac{G(k)}{\left(|k|^{2}+1\right)^{s / 2}} e^{2 \pi i k \cdot a}$ converges absolutely and defines a holomorphic function in the half plane $\{\sigma>-N\}$. Therefore, for any $s \in\{\operatorname{Re}(s)>-N\}$ we have

$$
\left|F_{1}(G ; a ; s)\right| \ll_{G} \sum_{k \in \mathbb{Z}^{n}} \frac{1}{\left(|k|^{2}+1\right)^{\left(-N+g_{0}\right) / 2}} \ll_{G} \sum_{k \in \mathbb{Z}^{n}} \frac{1}{\left(|k|^{2}+1\right)^{(n+1) / 2}} \ll_{G} 1 .
$$

Thus, Lemma 2.7 is true when $\operatorname{deg}(G) \leq-g_{0}$.

Step 2: Induction.

Now let $g \in \mathbb{Z}$ satisfy $g \geq-g_{0}+1$ and suppose that Lemma 2.7 is valid for all $G \in \mathcal{F}$ with $\operatorname{deg}(G) \leq g-1$. Let $G \in \mathcal{F}$ with $\operatorname{deg}(G)=g$. We will prove that $G$ also fulfills the conclusions of Lemma 2.7:

There exist $P \in \mathbb{C}\left[X_{1}, \ldots, X_{n}\right]$ of degree $d \geq 0$ and $r \in \mathbb{N}_{0}$ such that $G(X)=$ $\frac{P(X)}{\left(X_{1}^{2}+\cdots+X_{n}^{2}+1\right)^{r / 2}}$ and $g=\operatorname{deg}(G)=d-r$. Since $G(k) \ll\left(|k|^{2}+1\right)^{g / 2}$ uniformly in $k \in \mathbb{Z}^{n}$, we deduce that $F_{1}(G ; a ; s)$ converges absolutely in $\{\sigma=\operatorname{Re}(s)>n+g\}$.

Since $k \mapsto k+u$ is a bijection from $\mathbb{Z}^{n}$ into $\mathbb{Z}^{n}$, it follows that for $\operatorname{Re}(s)>n+g$ we also have

$$
\begin{aligned}
& F_{1}(G ; a ; s) \\
& =\sum_{k \in \mathbb{Z}^{n}} \frac{P(k)}{\left(|k|^{2}+1\right)^{(s+r) / 2}} e^{2 \pi i k \cdot a} \\
& =\sum_{k \in \mathbb{Z}^{n}} \frac{P(k+u)}{\left(|k+u|^{2}+1\right)^{(s+r) / 2}} e^{2 \pi i(k+u) \cdot a} \\
& =e^{2 \pi i u \cdot a} \sum_{k \in \mathbb{Z}^{n}} \frac{P(k+u)}{\left(|k|^{2}+2 k \cdot u+|u|^{2}+1\right)^{(s+r) / 2}} e^{2 \pi i k \cdot a} \partial^{\alpha} P(k) \\
& =e^{2 \pi i u \cdot a} \sum_{\substack{\alpha \in \mathbb{N}_{0}^{n} \\
|\alpha|_{1}=\alpha_{1}+\cdots+\alpha_{n} \leq d}} \frac{u^{\alpha}}{\alpha !} \sum_{k \in \mathbb{Z}^{n}} \frac{\left(|k|^{2}+2 k \cdot u+|u|^{2}+1\right)^{(s+r) / 2}}{2 \pi i k \cdot a}=
\end{aligned}
$$




$$
=e^{2 \pi i u \cdot a} \sum_{|\alpha|_{1} \leq d} \frac{u^{\alpha}}{\alpha !} \sum_{k \in \mathbb{Z}^{n}} \frac{\partial^{\alpha} P(k)}{\left(|k|^{2}+1\right)^{(s+r) / 2}}\left(1+\frac{2 k \cdot u+|u|^{2}}{\left(|k|^{2}+1\right)}\right)^{-(s+r) / 2} e^{2 \pi i k \cdot a} .
$$

Let $M:=\sup (N+n+g, 0) \in \mathbb{N}_{0}$. We have uniformly in $k \in \mathbb{Z}^{n}$ :

$$
\begin{aligned}
\left(1+\frac{2 k \cdot u+|u|^{2}}{\left(|k|^{2}+1\right)}\right)^{-(s+r) / 2}=\sum_{j=0}^{M} & \left(\begin{array}{c}
-(s+r) / 2 \\
j
\end{array}\right) \frac{\left(2 k \cdot u+|u|^{2}\right)^{j}}{\left(|k|^{2}+1\right)^{j}} \\
& +O_{M, u}\left(\frac{(1+|s|)^{M+1}}{\left(|k|^{2}+1\right)^{(M+1) / 2}}\right) .
\end{aligned}
$$

Thus for $\sigma=\operatorname{Re}(s)>n+d$ it follows that

$$
\begin{aligned}
& F_{1}(G ; a ; s) \\
& =e^{2 \pi i u \cdot a} \sum_{|\alpha|_{1} \leq d} \frac{u^{\alpha}}{\alpha !} \sum_{k \in \mathbb{Z}^{n}} \frac{\partial^{\alpha} P(k)}{\left(|k|^{2}+1\right)^{(s+r) / 2}}\left(1+\frac{2 k \cdot u+|u|^{2}}{\left(|k|^{2}+1\right)}\right)^{-(s+r) / 2} e^{2 \pi i k \cdot a} \\
& =e^{2 \pi i u \cdot a} \sum_{|\alpha|_{1} \leq d} \sum_{j=0}^{M} \frac{u^{\alpha}}{\alpha !}\left(\begin{array}{c}
-(s+r) / 2 \\
j
\end{array}\right) \sum_{k \in \mathbb{Z}^{n}} \frac{\partial^{\alpha} P(k)\left(2 k \cdot u+|u|^{2}\right)^{j}}{\left(|k|^{2}+1\right)^{(s+r+2 j) / 2}} e^{2 \pi i k \cdot a} \\
& \quad+O_{G, M, u}\left((1+|s|)^{M+1} \sum_{k \in \mathbb{Z}^{n}} \frac{1}{\left(|k|^{2}+1\right)^{(\sigma+M+1-g) / 2}}\right) .
\end{aligned}
$$

Set $I:=\left\{(\alpha, j) \in \mathbb{N}_{0}^{n} \times\left.\{0, \ldots, M\}|| \alpha\right|_{1} \leq d\right\}$ and $I^{*}:=I \backslash\{(0,0)\}$. Set also $G_{(\alpha, j) ; u}(X):=\frac{\partial^{\alpha} P(X)\left(2 X \cdot u+|u|^{2}\right)^{j}}{\left(|X|^{2}+1\right)^{(r+2 j) / 2}} \in \mathcal{F}$ for all $(\alpha, j) \in I^{*}$.

Since $M \geq N+n+g$, it follows from (2.3) that

$$
\begin{gathered}
\left(1-e^{2 \pi i u \cdot a}\right) F_{1}(G ; a ; s)=e^{2 \pi i u \cdot a} \sum_{(\alpha, j) \in I^{*}} \frac{u^{\alpha}}{\alpha !}\left(\begin{array}{c}
-(s+r) / 2 \\
j
\end{array}\right) F_{1}\left(G_{(\alpha, j) ; u} ; \alpha ; s\right) \\
+R_{N}(G ; a ; u ; s),
\end{gathered}
$$

where the map $s \mapsto R_{N}(G ; a ; u ; s)$ is a holomorphic function in the half plane $\{\sigma=\operatorname{Re}(s)>-N\}$ satisfying $R_{N}(G ; a ; u ; s) \ll_{G, N, u} 1$.

Moreover it is easy to see that for any $(\alpha, j) \in I^{*}$,

$$
\begin{aligned}
\operatorname{deg}\left(G_{(\alpha, j) ; u}\right) & =\operatorname{deg}\left(\partial^{\alpha} P\right)+j-(r+2 j) \\
& \leq d-|\alpha|_{1}+j-(r+2 j)=g-|\alpha|_{1}-j \leq g-1 .
\end{aligned}
$$

Relation (2.4) and the induction hypothesis then imply that

$$
\left(1-e^{2 \pi i u \cdot a}\right) F_{1}(G ; a ; s) \text { verifies the conclusions of Lemma 2.7. }
$$

Since $\left|1-e^{2 \pi i u \cdot a}\right|=2|\sin (\pi u \cdot a)| \geq d(u \cdot a, \mathbb{Z}),(2.5)$ implies that $F_{1}(G ; a ; s)$ satisfies the conclusions of Lemma 2.7. This completes the induction and the proof for $i=1$. 
2.2.2. Proof of Lemma 2.7 for $\boldsymbol{i}=\mathbf{0}$. Let $N \in \mathbb{N}$ be a fixed integer. Let $G(X)=$ $\frac{P(X)}{\left(X_{1}^{2}+\cdots+X_{n}^{2}+1\right)^{r / 2}} \in \mathcal{F}$ and $g=\operatorname{deg}(G)=d-r$ where $d \geq 0$ is the degree of the polynomial $P$. Put $M:=\sup (N+g+n, 0) \in \mathbb{N}_{0}$.

Since $P(k) \ll|k|^{d}$ for $k \in \mathbb{Z}^{n} \backslash\{0\}$, it follows that $F_{0}(G ; a ; s)$ and $F_{1}(G ; a ; s)$ converge absolutely in the half plane $\{\sigma=\operatorname{Re}(s)>n+g\}$. Moreover, we have for $s=\sigma+i \tau \in \mathbb{C}$ with $\sigma>n+g$ :

$$
\begin{aligned}
F_{0}(G ; a ; s)= & \sum_{k \in \mathbb{Z}^{n} \backslash\{0\}} \frac{G(k)}{\left(|k|^{2}+1-1\right)^{s / 2}} e^{2 \pi i k \cdot a} \\
= & \sum_{k \in \mathbb{Z}^{n}}^{\prime} \frac{G(k)}{\left(|k|^{2}+1\right)^{s / 2}}\left(1-\frac{1}{|k|^{2}+1}\right)^{-s / 2} e^{2 \pi i k \cdot a} \\
= & \sum_{k \in \mathbb{Z}^{n}}^{\prime} \sum_{j=0}^{M}\left(\begin{array}{c}
-s / 2 \\
j
\end{array}\right)(-1)^{j} \frac{G(k)}{\left(|k|^{2}+1\right)^{(s+2 j) / 2}} e^{2 \pi i k \cdot a} \\
& +O_{M}\left((1+|s|)^{M+1} \sum_{k \in \mathbb{Z}^{n}}^{\prime} \frac{|G(k)|}{\left(|k|^{2}+1\right)^{(\sigma+2 M+2) / 2}}\right) \\
= & \sum_{j=0}^{M}\left(\begin{array}{c}
-s / 2 \\
j
\end{array}\right)(-1)^{j} F_{1}(G ; a ; s+2 j) \\
& +O_{M}\left[(1+|s|)^{M+1}\left(1+\sum_{k \in \mathbb{Z}^{n}}^{\prime} \frac{|G(k)|}{\left(|k|^{2}+1\right)^{(\sigma+2 M+2) / 2}}\right)\right] .
\end{aligned}
$$

In addition we have uniformly in $s=\sigma+i \tau \in \mathbb{C}$ with $\sigma>-N$,

$$
\begin{aligned}
\sum_{k \in \mathbb{Z}^{n}}^{\prime} \frac{|G(k)|}{\left(|k|^{2}+1\right)^{(\sigma+2 M+2) / 2}} & \ll \sum_{k \in \mathbb{Z}^{n}}^{\prime} \frac{|k|^{g}}{\left(|k|^{2}+1\right)^{(-N+2 M+2) / 2}} \\
& \ll \sum_{k \in \mathbb{Z}^{n}}^{\prime} \frac{1}{|k|^{n+1}}<+\infty .
\end{aligned}
$$

So (2.6) and Lemma 2.7 for $i=1$ imply that Lemma 2.7 is also true for $i=0$. This completes the proof of Lemma 2.7.

2.2.3. Proof of item (i.2) of Theorem 2.6. Since $a \in \mathbb{R}^{n} \backslash \mathbb{Z}^{n}$, there exists $i_{0} \in\{1, \ldots, n\}$ such that $a_{i_{0}} \notin \mathbb{Z}$. In particular $d\left(a \cdot e_{i_{0}}, \mathbb{Z}\right)=d\left(a_{i_{0}}, \mathbb{Z}\right)>0$. Therefore, $a$ satisfies the assumption of Lemma 2.7 with $u=e_{i_{0}}$. Thus, for all $N \in \mathbb{N}, s \mapsto f_{a}(s)=F_{0}(P ; a ; s)$ has a holomorphic continuation to the half plane $\{\operatorname{Re}(s)>-N\}$. It follows, by letting $N \rightarrow \infty$, that $s \mapsto f_{a}(s)$ has a holomorphic continuation to the whole complex plane $\mathbb{C}$. 
2.2.4. Proof of item (iii) of Theorem 2.6. Let $\Theta \in \mathcal{M}_{n}(\mathbb{R}),\left(\varepsilon_{i}\right)_{i} \in\{-1,0,1\}^{q}$ and $b \in \mathcal{S}\left(\mathbb{Z}^{n} \times \mathbb{Z}^{n}\right)$. We assume that $\Theta$ is a diophantine matrix. Set $z:=$ $\left\{l=\left(l_{1}, \ldots, l_{q}\right) \in\left(\mathbb{Z}^{n}\right)^{q} \mid \sum_{i} \varepsilon_{i} l_{i}=0\right\}$ and $P \in \mathbb{C}\left[X_{1}, \ldots, X_{n}\right]$ of degree $d \geq 0$.

It is easy to see that for $\sigma>n+d$ :

$$
\begin{aligned}
\sum_{l \in\left(\mathbb{Z}^{n}\right)^{q} \backslash z}|b(l)| \sum_{k \in \mathbb{Z}^{n}}^{\prime} \frac{|P(k)|}{|k|^{\sigma}}\left|e^{2 \pi i k \cdot \Theta \sum_{i} \varepsilon_{i} l_{i}}\right| & \ll_{P} \sum_{l \in\left(\mathbb{Z}^{n}\right)^{q} \backslash z}|b(l)| \sum_{k \in \mathbb{Z}^{n}}^{\prime} \frac{1}{|k|^{\sigma-d}} \\
& \ll_{P, \sigma} \sum_{l \in\left(\mathbb{Z}^{n}\right)^{q} \backslash z}|b(l)|<+\infty .
\end{aligned}
$$

So

$$
g_{0}(s):=\sum_{l \in\left(\mathbb{Z}^{n}\right)^{q} \backslash z} b(l) f_{\Theta \sum_{i} \varepsilon_{i} l_{i}}(s)=\sum_{l \in\left(\mathbb{Z}^{n}\right)^{q} \backslash z} b(l) \sum_{k \in \mathbb{Z}^{n}}^{\prime} \frac{P(k)}{|k|^{s}} e^{2 \pi i k \cdot \Theta \sum_{i} \varepsilon_{i} l_{i}}
$$

converges absolutely in the half plane $\{\operatorname{Re}(s)>n+d\}$.

Moreover with the notations of Lemma 2.7, we have for all $s=\sigma+i \tau \in \mathbb{C}$ with $\sigma>n+d$ :

$$
g_{0}(s)=\sum_{l \in\left(\mathbb{Z}^{n}\right)^{q} \backslash z} b(l) f_{\Theta \sum_{i} \varepsilon_{i} l_{i}}(s)=\sum_{l \in\left(\mathbb{Z}^{n}\right)^{q} \backslash z} b(l) F_{0}\left(P ; \Theta \sum_{i} \varepsilon_{i} l_{i} ; s\right) .
$$

But $\Theta$ is diophantine, so there exist $u \in \mathbb{Z}^{n}$ and $\delta, c>0$ such

$$
\left|q \cdot{ }^{t} \Theta u-m\right| \geq c(1+|q|)^{-\delta} \quad \text { for all } q \in \mathbb{Z}^{n} \backslash\{0\} \text { and for all } m \in \mathbb{Z} .
$$

We deduce that for all $l \in\left(\mathbb{Z}^{n}\right)^{q} \backslash Z$,

$\left|\left(\Theta \sum_{i} \varepsilon_{i} l_{i}\right) \cdot u-m\right|=\left|\left(\sum_{i} \varepsilon_{i} l_{i}\right) \cdot{ }^{t} \Theta u-m\right| \geq c\left(1+\left|\sum_{i} \varepsilon_{i} l_{i}\right|\right)^{-\delta} \geq c(1+|l|)^{-\delta}$.

It follows that there exist $u \in \mathbb{Z}^{n}, \delta>0$ and $c>0$ such that

$$
d\left(\left(\Theta \sum_{i} \varepsilon_{i} l_{i}\right) \cdot u ; \mathbb{Z}\right) \geq c(1+|l|)^{-\delta} \quad \text { for all } l \in\left(\mathbb{Z}^{n}\right)^{q} \backslash Z .
$$

Therefore, for any $l \in\left(\mathbb{Z}^{n}\right)^{q} \backslash Z$, the vector $a=\Theta \sum_{i} \varepsilon_{i} l_{i}$ verifies the assumption of Lemma 2.7 with the same $u$. Moreover $\delta$ and $c$ in (2.8) are also independent on $l$.

We fix now $N \in \mathbb{N}$. Lemma 2.7 implies that there exist positive constants $C_{0}:=C_{0}(P, N, u), B_{0}:=B_{i}(P, N, u)$ and $A_{0}:=A_{0}(P, N, u)$ such that for all $l \in\left(\mathbb{Z}^{n}\right)^{q} \backslash Z$, the map $s \mapsto F_{0}\left(P ; \Theta \sum_{i} \varepsilon_{i} l_{i} ; s\right)$ extends holomorphically to the half plane $\{\operatorname{Re}(s)>-N\}$ satisfying

$$
F_{0}\left(P ; \Theta \sum_{i} \varepsilon_{i} l_{i} ; s\right) \leq C_{0}(1+|s|)^{B_{0}} d\left(\left(\Theta \sum_{i} \varepsilon_{i} l_{i}\right) \cdot u ; \mathbb{Z}\right)^{-A_{0}}
$$


Together with (2.8) this implies that for any compact set $K$ included in the half plane $\{\operatorname{Re}(s)>-N\}$, there exist two constants $C:=C(P, N, c, \delta, u, K)$ and $D:=$ $D(P, N, c, \delta, u)$ (independent of $\left.l \in\left(\mathbb{Z}^{n}\right)^{q} \backslash Z\right)$ such that

$$
F_{0}\left(P ; \Theta \sum_{i} \varepsilon_{i} l_{i} ; s\right) \leq C(1+|l|)^{D} \quad \text { for all } s \in K \text { and for all } l \in\left(\mathbb{Z}^{n}\right)^{q} \backslash Z \text {. (2.9) }
$$

It follows that $s \mapsto \sum_{l \in\left(\mathbb{Z}^{n}\right)^{q} \backslash Z} b(l) F_{0}\left(P ; \Theta \sum_{i} \varepsilon_{i} l_{i} ; s\right)$ has a holomorphic continuation to the half plane $\{\operatorname{Re}(s)>-N\}$.

This and (2.7) imply that $s \mapsto g_{0}(s)=\sum_{l \in\left(\mathbb{Z}^{n}\right)^{q} \backslash z} b(l) f_{\Theta \sum_{i} \varepsilon_{i} l_{i}}(s)$ has a holomorphic continuation to $\{\operatorname{Re}(s)>-N\}$. Since $N$ is an arbitrary integer, by letting $N \rightarrow \infty$, it follows that $s \mapsto g_{0}(s)$ has a holomorphic continuation to the whole complex plane $\mathbb{C}$, which completes the proof of the theorem.

Remark 2.9. From equation (2.5) we see that a Diophantine condition is sufficient to get Lemma 2.7. Our Diophantine condition appears also (in equivalent form) in Connes [6], Prop. 49 (see Remark 4.2). The following heuristic argument shows that our condition seems to be necessary in order to get the result of Theorem 2.6:

For simplicity we assume $n=1$ (but the argument extends easily to any $n$ ). Let $\theta \in \mathbb{R} \backslash \mathbb{Q}$. We know (see this reflection formula in [15], p. 6) that for any $l \in \mathbb{Z} \backslash\{0\}$,

$$
g_{\theta l}(s):=\sum_{k \in \mathbb{Z}}^{\prime} \frac{e^{2 \pi i \theta l k}}{|k|^{s}}=\frac{\pi^{s-1 / 2}}{\Gamma\left(\frac{1-s}{2}\right)} \Gamma\left(\frac{s}{2}\right) h_{\theta l}(1-s)
$$

where $h_{\theta l}(s):=\sum_{k \in \mathbb{Z}}^{\prime} \frac{1}{|\theta l+k|^{s}}$. So, for any $\left(a_{l}\right) \in \mathcal{S}(\mathbb{Z})$, the existence of a meromorphic continuation of $g_{0}(s):=\sum_{l \in \mathbb{Z}}^{\prime} a_{l} g_{\theta l}(s)$ is equivalent to the existence of a meromorphic continuation of

$$
h_{0}(s):=\sum_{l \in \mathbb{Z}}^{\prime} a_{l} h_{\theta l}(s)=\sum_{l \in \mathbb{Z}}^{\prime} a_{l} \sum_{k \in \mathbb{Z}}^{\prime} \frac{1}{|\theta l+k|^{s}} .
$$

So, for at least one $\sigma_{0} \in \mathbb{R}$, we must have $\frac{\left|a_{l}\right|}{|\theta l+k|^{\sigma_{0}}}=O(1)$ uniformly in $k, l \in \mathbb{Z}^{*}$.

It follows that for any $\left(a_{l}\right) \in \mathcal{S}(\mathbb{Z}),|\theta l+k| \gg\left|a_{l}\right|^{1 / \sigma_{0}}$ uniformly in $k, l \in \mathbb{Z}^{*}$. Therefore, our Diophantine condition seems to be necessary.

2.2.5. Commutation between sum and residue. Let $p \in \mathbb{N}$. Recall that $S\left(\left(\mathbb{Z}^{n}\right)^{p}\right)$ is the set of the Schwartz sequences on $\left(\mathbb{Z}^{n}\right)^{p}$. In other words, $b \in \mathcal{S}\left(\left(\mathbb{Z}^{n}\right)^{p}\right)$ if and only if $\left(1+\left|l_{1}\right|^{2}+\ldots\left|l_{p}\right|^{2}\right)^{r}\left|b\left(l_{1}, \ldots, l_{p}\right)\right|^{2}$ is bounded on $\left(\mathbb{Z}^{n}\right)^{p}$ for all $r \in \mathbb{N}_{0}$. We note that if $Q \in \mathbb{R}\left[X_{1}, \ldots, X_{n p}\right]$ is a polynomial, $\left(a_{j}\right) \in \mathcal{S}\left(\mathbb{Z}^{n}\right)^{p}, b \in \mathcal{S}\left(\mathbb{Z}^{n}\right)$ and $\phi$ is a real-valued function, then $l:=\left(l_{1}, \ldots, l_{p}\right) \mapsto \tilde{a}(l) b\left(-\hat{l}_{p}\right) Q(l) e^{i \phi(l)}$ is a Schwartz sequence on $\left(\mathbb{Z}^{n}\right)^{p}$, where

$$
\tilde{a}(l):=a_{1}\left(l_{1}\right) \ldots a_{p}\left(l_{p}\right), \quad \hat{l}_{i}:=l_{1}+\cdots+l_{i} .
$$


In the following, we will use several times the fact that for any $(k, l) \in\left(\mathbb{Z}^{n}\right)^{2}$ such that $k \neq 0$ and $k \neq-l$, we have

$$
\frac{1}{|k+l|^{2}}=\frac{1}{|k|^{2}}-\frac{2 k \cdot l+|l|^{2}}{|k|^{2}|k+l|^{2}} .
$$

Lemma 2.10. There exists a polynomial $P \in \mathbb{R}\left[X_{1}, \ldots, X_{p}\right]$ of degree $4 p$ and with positive coefficients such that for any $k \in \mathbb{Z}^{n}$ and $l:=\left(l_{1}, \ldots, l_{p}\right) \in\left(\mathbb{Z}^{n}\right)^{p}$ such that $k \neq 0$ and $k \neq-\hat{l}_{i}$ for all $1 \leq i \leq p$, the following holds:

$$
\frac{1}{\left|k+\hat{l}_{1}\right|^{2} \ldots\left|k+\hat{l}_{p}\right|^{2}} \leq \frac{1}{|k|^{2 p}} P\left(\left|l_{1}\right|, \ldots,\left|l_{p}\right|\right) .
$$

Proof. Let us fix $i$ such that $1 \leq i \leq p$. Using two times (2.10), the Cauchy-Schwarz inequality and the fact that $\left|k+\hat{l}_{i}\right|^{2} \geq 1$, we get

$$
\begin{aligned}
\frac{1}{\left|k+\hat{l}_{i}\right|^{2}} & \leq \frac{1}{|k|^{2}}+\frac{2|k|\left|\hat{l}_{i}\right|+\left|\hat{l}_{i}\right|^{2}}{|k|^{4}}+\frac{\left(2|k|\left|\hat{l}_{i}\right|+\left|\hat{l}_{i}\right|^{2}\right)^{2}}{|k|^{4}\left|k+\hat{l}_{i}\right|^{2}} \\
& \leq \frac{1}{|k|^{2}}+\frac{2}{|k|^{3}}\left|\hat{l}_{i}\right|+\left(\frac{1}{|k|^{4}}+\frac{4}{|k|^{2}}\right)\left|\hat{l}_{i}\right|^{2}+\frac{4}{|k|^{3}}\left|\hat{l}_{i}\right|^{3}+\frac{1}{|k|^{4}}\left|\hat{l}_{i}\right|^{4} .
\end{aligned}
$$

Since $|k| \geq 1$, and $\left|\hat{l}_{i}\right|^{j} \leq\left|\hat{l}_{i}\right|^{4}$ if $1 \leq j \leq 4$, we find

$$
\begin{gathered}
\frac{1}{\left|k+\hat{l}_{i}\right|^{2}} \leq \frac{5}{|k|^{2}} \sum_{j=0}^{4}\left|\hat{l}_{i}\right|^{j} \leq \frac{5}{|k|^{2}}\left(1+4\left|\hat{l}_{i}\right|^{4}\right) \leq \frac{5}{|k|^{2}}\left(1+4\left(\sum_{j=1}^{p}\left|l_{j}\right|\right)^{4}\right), \\
\frac{1}{\left|k+\hat{l}_{1}\right|^{2} \ldots\left|k+\hat{l}_{p}\right|^{2}} \leq \frac{5^{p}}{|k|^{2 p}}\left(1+4\left(\sum_{j=1}^{p}\left|l_{j}\right|\right)^{4}\right)^{p} .
\end{gathered}
$$

Taking $P\left(X_{1}, \ldots, X_{p}\right):=5^{p}\left(1+4\left(\sum_{j=1}^{p} X_{j}\right)^{4}\right)^{p}$ now gives the result.

Lemma 2.11. Let $b \in \mathcal{S}\left(\left(\mathbb{Z}^{n}\right)^{p}\right), p \in \mathbb{N}$. Let $P_{j} \in \mathbb{R}\left[X_{1}, \ldots, X_{n}\right]$ be a homogeneous polynomial function of degree $j$, and let $k \in \mathbb{Z}^{n}, l:=\left(l_{1}, \ldots, l_{p}\right) \in\left(\mathbb{Z}^{n}\right)^{p}, r \in \mathbb{N}_{0}$. Moreover, let $\phi$ be a real-valued function on $\mathbb{Z}^{n} \times\left(\mathbb{Z}^{n}\right)^{p}$ and put

$$
h(s, k, l):=\frac{b(l) P_{j}(k) e^{i \phi(k, l)}}{|k|^{s+r}\left|k+\hat{l}_{1}\right|^{2} \ldots\left|k+\hat{l}_{p}\right|^{2}},
$$

with $h(s, k, l):=0$ if, for $k \neq 0$, one of the denominators is zero.

Then for all $s \in \mathbb{C}$ such that $\operatorname{Re}(s)>n+j-r-2 p$, the series

$$
H(s):=\sum_{(k, l) \in\left(\mathbb{Z}^{n}\right)^{p+1}}^{\prime} h(s, k, l)
$$


is absolutely summable. In particular,

$$
\sum_{k \in \mathbb{Z}^{n}}^{\prime} \sum_{l \in\left(\mathbb{Z}^{n}\right)^{p}} h(s, k, l)=\sum_{l \in\left(\mathbb{Z}^{n}\right)^{p}} \sum_{k \in \mathbb{Z}^{n}}^{\prime} h(s, k, l) .
$$

Proof. Let $s=\sigma+i \tau \in \mathbb{C}$ such that $\sigma>n+j-r-2 p$. By Lemma 2.10 we obtain, for $k \neq 0$, that

$$
|h(s, k, l)| \leq\left|b(l) P_{j}(k)\right||k|^{-r-\sigma-2 p} P(l),
$$

where $P(l):=P\left(\left|l_{1}\right|, \ldots,\left|l_{p}\right|\right)$ and $P$ is a polynomial of degree $4 p$ with positive coefficients. Thus, $|h(s, k, l)| \leq F(l) G(k)$ where $F(l):=|b(l)| P(l)$ and $G(k):=$ $\left|P_{j}(k)\right||k|^{-r-\sigma-2 p}$. That $\sum_{l \in\left(\mathbb{Z}^{n}\right)^{p}} F(l)$ is summable follows from the fact that $b \in \mathcal{S}\left(\left(\mathbb{Z}^{n}\right)^{p}\right)$. The summability of $\sum_{k \in \mathbb{Z}^{n}}^{\prime} G(k)$ is a consequence of the fact that $\sigma>n+j-r-2 p$. Finally, as a product of two summable series, $\sum_{k, l} F(l) G(k)$ is a summable series, which proves that $\sum_{k, l} h(s, k, l)$ is also absolutely summable.

Definition 2.12. Let $f$ be a function on $D \times\left(\mathbb{Z}^{n}\right)^{p}$ where $D$ is an open neighborhood of 0 in $\mathbb{C}$. We say that $f$ satisfies (H1) if and only if there exists $\rho>0$ such that

(i) for any $l, s \mapsto f(s, l)$ extends as a holomorphic function in $U_{\rho}$, where $U_{\rho}$ is the open disk of center 0 and radius $\rho$;

(ii) the series $\sum_{l \in\left(\mathbb{Z}^{n}\right)^{p}}\|H(\cdot, l)\|_{\infty, \rho}$ is summable, where $\|H(\cdot, l)\|_{\infty, \rho}:=$ $\sup _{s \in U_{\rho}}|H(s, l)|$.

We say that $f$ satisfies (H2) if and only if there exists $\rho>0$ such that

(i) for any $l, s \mapsto f(s, l)$ extends as a holomorphic function to $U_{\rho}-\{0\}$;

(ii) for any $\delta$ such that $0<\delta<\rho$, the series $\sum_{l \in\left(\mathbb{Z}^{n}\right)^{p}}\|H(\cdot, l)\|_{\infty, \delta, \rho}$ is summable, where $\|H(\cdot, l)\|_{\infty, \delta, \rho}:=\sup _{\delta<|s|<\rho}|H(s, l)|$.

Remark 2.13. Note that (H1) implies (H2). Moreover, if $f$ satisfies (H1) (resp. (H2)) for $\rho>0$, then it is straightforward to check that $f: s \mapsto \sum_{l \in\left(\mathbb{Z}^{n}\right)^{p}} f(s, l)$ extends as an holomorphic function to $U_{\rho}$ (resp. to $U_{\rho} \backslash\{0\}$ ).

Corollary 2.14. With the same notations as in Lemma 2.11, suppose that $r+2 p-j>$ $n$. Then the function $H(s, l):=\sum_{k \in \mathbb{Z}^{n}}^{\prime} h(s, k, l)$ satisfies $(\mathrm{H} 1)$.

Proof. (i) Fix $\rho>0$ such that $\rho<r+2 p-j-n$. Since $r+2 p-j>n, U_{\rho}$ is inside the half plane of absolute convergence of the series defined by $H(s, l)$. Thus, $s \mapsto H(s, l)$ is holomorphic in $U_{\rho}$.

(ii) Since $\left.\left.|| k\right|^{-s}|\leq| k\right|^{\rho}$ for all $s \in U_{\rho}$ and $k \in \mathbb{Z}^{n} \backslash\{0\}$, we get as in the above proof

$$
|h(s, k, l)| \leq\left|b(l) P_{j}(k)\right||k|^{-r+\rho-2 p} P\left(\left|l_{1}\right|, \ldots,\left|l_{p}\right|\right) .
$$


Since $\rho<r+2 p-j-n$, the series $\sum_{k \in \mathbb{Z}^{n}}^{\prime}\left|P_{j}(k)\right||k|^{-r+\rho-2 p}$ is summable.

Thus, $\|H(\cdot, l)\|_{\infty, \rho} \leq K F(l)$ where $K:=\sum_{k}{ }^{\prime}\left|P_{j}(k) \| k\right|^{-r+\rho-2 p}<\infty$. We have already seen that the series $\sum_{l} F(l)$ is summable, so the result follows.

We note that if $f$ and $g$ both satisfy (H1) (or (H2)), then so does $f+g$. In the following we will use the equivalence relation

$$
f \sim g \Longleftrightarrow f-g \text { satisfies (H1). }
$$

Lemma 2.15. Let $f$ and $g$ be two functions on $D \times\left(\mathbb{Z}^{n}\right)^{p}$, where $D$ is an open neighborhood of 0 , in $\mathbb{C}$ such that $f \sim g$ and $g$ satisfies $(\mathrm{H} 2)$. Then

$$
\operatorname{Res}_{s=0} \sum_{l \in\left(\mathbb{Z}^{n}\right)^{p}} f(s, l)=\sum_{l \in\left(\mathbb{Z}^{n}\right)^{p}} \operatorname{Res}_{s=0} g(s, l) .
$$

Proof. Since $f \sim g, f$ satisfies (H2) for a certain $\rho>0$. Fix $\eta$ such that $0<\eta<\rho$ and define $C_{\eta}$ as the circle of center 0 and radius $\eta$. Then

$$
\operatorname{Res}_{s=0} g(s, l)=\operatorname{Res}_{s=0} f(s, l)=\frac{1}{2 \pi i} \oint_{C_{n}} f(s, l) d s=\int_{I} u(t, l) d t,
$$

where $I=[0,2 \pi]$ and $u(t, l):=\frac{1}{2 \pi} \eta e^{i t} f\left(\eta e^{i t}, l\right)$. The fact that $f$ satisfies (H2) entails that the series $\sum_{l \in\left(\mathbb{Z}^{n}\right)^{p}}\|f(\cdot, l)\|_{\infty, C_{\eta}}$ is summable. Thus, since $\|u(\cdot, l)\|_{\infty}=$ $\frac{1}{2 \pi} \eta\|f(\cdot, l)\|_{\infty, C_{\eta}}$, the series $\sum_{l \in\left(\mathbb{Z}^{n}\right)^{p}}\|u(\cdot, l)\|_{\infty}$ is summable, and consequently $\int_{I} \sum_{l \in\left(\mathbb{Z}^{n}\right)^{p}} u(t, l) d t=\sum_{l \in\left(\mathbb{Z}^{n}\right)^{p}} \int_{I} u(t, l) d t$, which gives the result.

2.3. Computation of residues of zeta functions. Since we will have to compute residues of series, let us introduce the following notation.

\section{Definition 2.16.}

$$
\begin{aligned}
\zeta(s) & :=\sum_{n=1}^{\infty} n^{-s}, \\
Z_{n}(s) & :=\sum_{k \in \mathbb{Z}^{n}}^{\prime}|k|^{-s}, \\
\zeta_{p_{1}, \ldots, p_{n}}(s) & :=\sum_{k \in \mathbb{Z}^{n}}^{\prime} \frac{k_{1}^{p_{1}} \ldots k_{n}^{p_{n}}}{|k|^{s}} \quad \text { for } p_{i} \in \mathbb{N},
\end{aligned}
$$

where $\zeta(s)$ is the Riemann zeta function (see [25] or [14]).

By the symmetry $k \rightarrow-k$, it is clear that the functions $\zeta_{p_{1}, \ldots, p_{n}}$ all vanish for odd values of $p_{i}$. 
Let us now compute $\zeta_{0, \ldots, 0,1_{i}, 0 \ldots, 0,1_{j}, 0 \ldots, 0}(s)$ in terms of $Z_{n}(s)$ :

Since $\zeta_{0, \ldots, 0,1_{i}, 0 \ldots, 0,1_{j}, 0 \ldots, 0}(s)=A_{i}(s) \delta_{i j}$, exchanging the components $k_{i}$ and $k_{j}$ we get

$$
\zeta_{0, \ldots, 0,1_{i}, 0 \ldots, 0,1_{j}, 0 \ldots, 0}(s)=\frac{\delta_{i j}}{n} Z_{n}(s-2)
$$

Similarly,

$$
\sum_{\mathbb{Z}^{n}}^{\prime} \frac{k_{1}^{2} k_{2}^{2}}{|k|^{s+8}}=\frac{1}{n(n-1)} Z_{n}(s+4)-\frac{1}{n-1} \sum_{\mathbb{Z}^{n}}^{\prime} \frac{k_{1}^{4}}{|k|^{s+8}},
$$

but it is difficult to write explicitly $\zeta_{p_{1}, \ldots, p_{n}}(s)$ in terms of $Z_{n}(s-4)$ and other $Z_{n}(s-m)$ when at least four indices $p_{i}$ are non-zero.

When all $p_{i}$ are even, $\zeta_{p_{1}, \ldots, p_{n}}(s)$ is a non-zero series of fractions $\frac{P(k)}{|k|^{s}}$ where $P$ is a homogeneous polynomial of degree $p_{1}+\cdots+p_{n}$. Theorem 2.1 now gives us the following result.

Proposition 2.17. The function $\zeta_{p_{1}, \ldots, p_{n}}$ has a meromorphic extension to the whole plane with a unique pole at $n+p_{1}+\cdots+p_{n}$. This pole is simple and the residue at this pole is

$$
\operatorname{Res}_{s=n+p_{1}+\cdots+p_{n}} \zeta_{p_{1}, \ldots, p_{n}}(s)=2 \frac{\Gamma\left(\frac{p_{1}+1}{2}\right) \ldots \Gamma\left(\frac{p_{n}+1}{2}\right)}{\Gamma\left(\frac{n+p_{1}+\cdots+p_{n}}{2}\right)}
$$

when all $p_{i}$ are even or this residue is zero otherwise.

In particular, for $n=2$,

$$
\operatorname{Res}_{s=0} \sum_{k \in \mathbb{Z}^{2}}^{\prime} \frac{k_{i} k_{j}}{|k|^{s+4}}=\delta_{i j} \pi
$$

and for $n=4$,

$$
\begin{aligned}
& \operatorname{Res}_{s=0} \sum_{k \in \mathbb{Z}^{4}}^{\prime} \frac{k_{i} k_{j}}{|k|^{s+6}}=\delta_{i j} \frac{\pi^{2}}{2}, \\
& \operatorname{Res}_{s=0} \sum_{k \in \mathbb{Z}^{4}}^{\prime} \frac{k_{i} k_{j} k_{l} k_{m}}{|k|^{s+8}}=\left(\delta_{i j} \delta_{l m}+\delta_{i l} \delta_{j m}+\delta_{i m} \delta_{j l}\right) \frac{\pi^{2}}{12} .
\end{aligned}
$$

Proof. Equation (2.11) follows from Theorem 2.1,

$$
\operatorname{Res}_{s=n+p_{1}+\cdots+p_{n}} \zeta_{p_{1}, \ldots, p_{n}}(s)=\int_{k \in S^{n-1}} k_{1}^{p_{1}} \ldots k_{n}^{p_{n}} d S(k),
$$

and standard formulae (see for instance [32], VIII, 1; 22). Equation (2.12) is a straightforward consequence of equation (2.11). Equation (2.13) can be checked for the cases $i=j \neq l=m$ and $i=j=l=m$. 
Note that $Z_{n}(s)$ is an Epstein zeta function associated to the quadratic form $q(x):=x_{1}^{2}+\cdots+x_{n}^{2}$, so $Z_{n}$ satisfies the following functional equation

$$
Z_{n}(s)=\pi^{s-n / 2} \Gamma(n / 2-s / 2) \Gamma(s / 2)^{-1} Z_{n}(n-s) .
$$

Since $\pi^{s-n / 2} \Gamma(n / 2-s / 2) \Gamma(s / 2)^{-1}=0$ for any negative even integer $n$ and $Z_{n}(s)$ is meromorphic on $\mathbb{C}$ with only one pole at $s=n$ with residue $2 \pi^{n / 2} \Gamma(n / 2)^{-1}$ according to the previous proposition, it follows that $Z_{n}(0)=-1$. We have proved that

$$
\begin{aligned}
\operatorname{Res}_{s=0} Z_{n}(s+n) & =2 \pi^{n / 2} \Gamma(n / 2)^{-1}, \\
Z_{n}(0) & =-1 .
\end{aligned}
$$

2.4. Meromorphic continuation of a class of zeta functions. Let $n, q \in \mathbb{N}, q \geq 2$, and $p=\left(p_{1}, \ldots, p_{q-1}\right) \in \mathbb{N}_{0}^{q-1}$. Set $I:=\left\{i \mid p_{i} \neq 0\right\}$ and assume that $I \neq \emptyset$ and

$$
\mathcal{J}:=\left\{\alpha=\left(\alpha_{i}\right)_{i \in I} \mid \alpha_{i}=\left(\alpha_{i, 1}, \ldots, \alpha_{i, p_{i}}\right) \in \mathbb{N}_{0}^{p_{i}} \text { for all } i \in I\right\}=\prod_{i \in I} \mathbb{N}_{0}^{p_{i}} .
$$

We will use in the sequel also the following notations.

For $x=\left(x_{1}, \ldots, x_{t}\right) \in \mathbb{R}^{t}$ recall that $|x|_{1}=\left|x_{1}\right|+\cdots+\left|x_{t}\right|$ and $|x|=$ $\sqrt{x_{1}^{2}+\cdots+x_{t}^{2}}$

for all $\alpha=\left(\alpha_{i}\right)_{i \in I} \in \mathcal{J}=\prod_{i \in I} \mathbb{N}_{0}^{p_{i}}$ we have

$$
|\alpha|_{1}=\sum_{i \in I}\left|\alpha_{i}\right|_{1}=\sum_{i \in I} \sum_{j=1}^{p_{i}}\left|\alpha_{i, j}\right| \text { and }\left(\begin{array}{c}
1 / 2 \\
\alpha
\end{array}\right)=\prod_{i \in I}\left(\begin{array}{c}
1 / 2 \\
\alpha_{i}
\end{array}\right)=\prod_{i \in I} \prod_{j=1}^{p_{i}}\left(\begin{array}{c}
1 / 2 \\
\alpha_{i, j}
\end{array}\right) \text {. }
$$

2.4.1. A family of polynomials. In this paragraph we define a family of polynomials which plays an important role later. Consider first the variables:

- for $X_{1}, \ldots, X_{n}$ we set $X=\left(X_{1}, \ldots, X_{n}\right)$;

- for any $i=1, \ldots, 2 q$, we regard the variables $Y_{i, 1}, \ldots, Y_{i, n}$ and put $Y_{i}:=$ $\left(Y_{i, 1}, \ldots, Y_{i, n}\right)$ and $Y:=\left(Y_{1}, \ldots, Y_{2 q}\right)$;

- for $Y=\left(Y_{1}, \ldots, Y_{2 q}\right)$ we set $\tilde{Y}_{j}:=Y_{1}+\cdots+Y_{j}+Y_{q+1}+\cdots+Y_{q+j}$ for any $1 \leq j \leq q$.

We define for all $\alpha=\left(\alpha_{i}\right)_{i \in I} \in \mathcal{J}=\prod_{i \in I} \mathbb{N}_{0}^{p_{i}}$ the polynomial

$$
P_{\alpha}(X, Y):=\prod_{i \in I} \prod_{j=1}^{p_{i}}\left(2\left\langle X, \tilde{Y}_{i}\right\rangle+\left|\tilde{Y}_{i}\right|^{2}\right)^{\alpha_{i, j}}
$$

It is clear that $P_{\alpha}(X, Y) \in \mathbb{Z}[X, Y], \operatorname{deg}_{X} P_{\alpha} \leq|\alpha|_{1}$ and $\operatorname{deg}_{Y} P_{\alpha} \leq 2|\alpha|_{1}$. 
Let us fix a polynomial $Q \in \mathbb{R}\left[X_{1}, \ldots, X_{n}\right]$ and write $d:=\operatorname{deg} Q$. For $\alpha \in \mathcal{J}$ we want to expand $P_{\alpha}(X, Y) Q(X)$ in homogeneous polynomials in $X$ and $Y$. Define

$$
L(\alpha):=\left\{\left.\beta \in \mathbb{N}_{0}^{(2 q+1) n}|| \beta\right|_{1}-d_{\beta} \leq 2|\alpha|_{1} \text { and } d_{\beta} \leq|\alpha|_{1}+d\right\}
$$

where $d_{\beta}:=\sum_{1}^{n} \beta_{i}$, and set

$$
\left(\begin{array}{c}
1 / 2 \\
\alpha
\end{array}\right) P_{\alpha}(X, Y) Q(X)=: \sum_{\beta \in L(\alpha)} c_{\alpha, \beta} X^{\beta} Y^{\beta}
$$

where $c_{\alpha, \beta} \in \mathbb{R}, X^{\beta}:=X_{1}^{\beta_{1}} \ldots X_{n}^{\beta_{n}}$ and $Y^{\beta}:=Y_{1,1}^{\beta_{n+1}} \ldots Y_{2 q, n}^{\beta_{(2 q+1) n}}$. By definition, $X^{\beta}$ is a homogeneous polynomial of degree in $X$ equals to $d_{\beta}$. Write

$$
M_{\alpha, \beta}(Y):=c_{\alpha, \beta} Y^{\beta} \text {. }
$$

2.4.2. Residues of a class of zeta functions. In this section we prove the following result that will be used in Proposition 5.4 for the computation of the spectrum dimension of the noncommutative torus.

Theorem 2.18. (i) Let $\frac{1}{2 \pi} \Theta$ be a diophantine matrix, and $\tilde{a} \in \mathcal{S}\left(\left(\mathbb{Z}^{n}\right)^{2 q}\right)$. Then

$$
s \mapsto f(s):=\sum_{l \in\left[\left(\mathbb{Z}^{n}\right)^{q}\right]^{2}} \tilde{a}_{l} \sum_{k \in \mathbb{Z}^{n}}^{\prime} \prod_{i=1}^{q-1}\left|k+\tilde{l}_{i}\right|^{p_{i}}|k|^{-s} Q(k) e^{i k \cdot \Theta \sum_{1}^{q} l_{j}}
$$

has a meromorphic continuation to the whole complex plane $\mathbb{C}$ with at most simple possible poles at the points $s=n+d+|p|_{1}-m$ where $m \in \mathbb{N}_{0}$.

(ii) Let $m \in \mathbb{N}_{0}$ and set $I(m):=\left\{(\alpha, \beta) \in \mathcal{J} \times \mathbb{N}_{0}^{(2 q+1) n} \mid \beta \in L(\alpha)\right.$ and $m=$ $\left.2|\alpha|_{1}-d_{\beta}+d\right\}$. Then $I(m)$ is a finite set and $s=n+d+|p|_{1}-m$ is a pole of $f$ if and only if

$$
C(f, m):=\sum_{l \in Z} \tilde{a}_{l} \sum_{(\alpha, \beta) \in I(m)} M_{\alpha, \beta}(l) \int_{u \in S^{n-1}} u^{\beta} d S(u) \neq 0,
$$

with $Z:=\left\{l \mid \sum_{1}^{q} l_{j}=0\right\}$ and the convention $\sum_{\emptyset}=0$. In that case $s=$ $n+d+|p|_{1}-m$ is a simple pole of residue $\underset{s=n+d+|p|_{1}-m}{\operatorname{Res}} f(s)=C(f, m)$.

In order to prove the theorem above we need the following result.

Lemma 2.19. For all $N \in \mathbb{N}$ we have

$\prod_{i=1}^{q-1}\left|k+\tilde{l}_{i}\right|^{p_{i}}=\sum_{\alpha=\left(\alpha_{i}\right)_{i \in I} \in \prod_{i \in I}\{0, \ldots, N\}^{p_{i}}}\left(\begin{array}{c}1 / 2 \\ \alpha\end{array}\right) \frac{P_{\alpha}(k, l)}{|k|^{|\alpha|_{1}-|p|_{1}}}+\mathcal{O}_{N}\left(|k|^{|p|_{1}-(N+1) / 2}\right)$ uniformly in $k \in \mathbb{Z}^{n}$ and $l \in\left(\mathbb{Z}^{n}\right)^{2 q}$ such that $|k|>U(l):=36\left(\sum_{i=1, i \neq q}^{2 q-1}\left|l_{i}\right|\right)^{4}$. 
Proof. For $i=1, \ldots, q-1$, we have uniformly in $k \in \mathbb{Z}^{n}$ and $l \in\left(\mathbb{Z}^{n}\right)^{2 q}$ with $|k|>U(l)$,

$$
\frac{\left.\left|2\left\langle k, \tilde{l}_{i}\right\rangle+\right| \tilde{l}_{i}\right|^{2} \mid}{|k|^{2}} \leq \frac{\sqrt{U(l)}}{2|k|}<\frac{1}{2 \sqrt{|k|}}
$$

In that case,

$$
\begin{aligned}
\left|k+\tilde{l}_{i}\right| & =\left(|k|^{2}+2\left\langle k, \tilde{l}_{i}\right\rangle+\left|\tilde{l}_{i}\right|^{2}\right)^{1 / 2}=|k|\left(1+\frac{2\left\langle k, \tilde{l}_{i}\right\rangle+\left|\tilde{l}_{i}\right|^{2}}{|k|^{2}}\right)^{1 / 2} \\
& =\sum_{u=0}^{\infty}\left(\begin{array}{c}
1 / 2 \\
u
\end{array}\right) \frac{1}{|k|^{2 u-1}} P_{u}^{i}(k, l)
\end{aligned}
$$

where

$$
P_{u}^{i}(k, l):=\left(2\left\langle k, \tilde{l}_{i}\right\rangle+\left|\tilde{l}_{i}\right|^{2}\right)^{u}
$$

for all $i=1, \ldots, q-1$ and for all $u \in \mathbb{N}_{0}$, and $P_{0}^{i}(k, l):=1$.

In particular $P_{u}^{i}(k, l) \in \mathbb{Z}[k, l], \operatorname{deg}_{k} P_{u}^{i} \leq u$ and $\operatorname{deg}_{l} P_{u}^{i} \leq 2 u$. Inequality (2.17) implies that for all $i=1, \ldots, q-1$ and for all $u \in \mathbb{N}$,

$$
\frac{1}{|k|^{2 u}}\left|P_{u}^{i}(k, l)\right| \leq(2 \sqrt{|k|})^{-u}
$$

uniformly in $k \in \mathbb{Z}^{n}$ and $l \in\left(\mathbb{Z}^{n}\right)^{2 q}$ such that $|k|>U(l)$.

Let $N \in \mathbb{N}$. We deduce from the previous that for any $k \in \mathbb{Z}^{n}$ and $l \in\left(\mathbb{Z}^{n}\right)^{2 q}$ with $|k|>U(l)$ and for all $i=1, \ldots, q-1$, we have

$$
\begin{aligned}
\left|k+\tilde{l}_{i}\right| & =\sum_{u=0}^{N}\left(\begin{array}{c}
1 / 2 \\
u
\end{array}\right) \frac{1}{|k|^{2 u-1}} P_{u}^{i}(k, l)+\mathcal{O}\left(\sum_{u>N}|k|\left|\left(\begin{array}{c}
1 / 2 \\
u
\end{array}\right)\right|(2 \sqrt{|k|})^{-u}\right) \\
& =\sum_{u=0}^{N}\left(\begin{array}{c}
1 / 2 \\
u
\end{array}\right) \frac{1}{|k|^{2 u-1}} P_{u}^{i}(k, l)+\mathcal{O}_{N}\left(\frac{1}{|k|^{(N-1) / 2}}\right) .
\end{aligned}
$$

It follows that for any $N \in \mathbb{N}$, we have uniformly in $k \in \mathbb{Z}^{n}$ and $l \in\left(\mathbb{Z}^{n}\right)^{2 q}$ with $|k|>U(l)$ and for all $i \in I$,

$$
\left|k+\tilde{l}_{i}\right|^{p_{i}}=\sum_{\alpha_{i} \in\{0, \ldots, N\}^{p_{i}}}\left(\begin{array}{c}
1 / 2 \\
\alpha_{i}
\end{array}\right) \frac{1}{|k|^{2\left|\alpha_{i}\right|_{1}-p_{i}}} P_{\alpha_{i}}^{i}(k, l)+\mathcal{O}_{N}\left(\frac{1}{|k|^{(N+1) / 2-p_{i}}}\right),
$$

where $P_{\alpha_{i}}^{i}(k, l)=\prod_{j=1}^{p_{i}} P_{\alpha_{i, j}}^{i}(k, l)$ for all $\alpha_{i}=\left(\alpha_{i, 1}, \ldots, \alpha_{i, p_{i}}\right) \in\{0, \ldots, N\}^{p_{i}}$ and

$$
\begin{aligned}
\prod_{i \in I}\left|k+\tilde{l}_{i}\right|^{p_{i}}= & \sum_{\alpha=\left(\alpha_{i}\right) \in \prod_{i \in I}\{0, \ldots, N\}^{p_{i}}}\left(\begin{array}{c}
1 / 2 \\
\alpha
\end{array}\right) \frac{1}{|k|^{2|\alpha|_{1}-|p|_{1}}} P_{\alpha}(k, l) \\
& +\mathcal{O}_{N}\left(\frac{1}{|k|^{(N+1) / 2-|p|_{1}}}\right)
\end{aligned}
$$


with $P_{\alpha}(k, l)=\prod_{i \in I} P_{\alpha_{i}}^{i}(k, l)=\prod_{i \in I} \prod_{j=1}^{p_{i}} P_{\alpha_{i, j}}^{i}(k, l)$.

Proof of Theorem 2.18. (i) All $n, q, p=\left(p_{1}, \ldots, p_{q-1}\right)$ and $\tilde{a} \in \mathcal{S}\left(\left(\mathbb{Z}^{n}\right)^{2 q}\right)$ are fixed as above and we define formally for any $l \in\left(\mathbb{Z}^{n}\right)^{2 q}$

$$
F(l, s):=\sum_{k \in \mathbb{Z}^{n}}^{\prime} \prod_{i=1}^{q-1}\left|k+\tilde{l}_{i}\right|^{p_{i}} Q(k) e^{i k \cdot \Theta \sum_{1}^{q} l_{j}}|k|^{-s} .
$$

Thus, still formally,

$$
f(s):=\sum_{l \in\left(\mathbb{Z}^{n}\right)^{2 q}} \tilde{a}_{l} F(l, s) .
$$

It is clear that $F(l, s)$ converges absolutely in the half plane $\{\sigma=\operatorname{Re}(s)>n+d+$ $\left.|p|_{1}\right\}$ where $d=\operatorname{deg} Q$.

Let $N \in \mathbb{N}$. Lemma 2.19 implies that for any $l \in\left(\mathbb{Z}^{n}\right)^{2 q}$ and for $s \in \mathbb{C}$ such that $\sigma>n+|p|_{1}+d$,

$$
\begin{aligned}
F(l, s)= & \sum_{|k| \leq U(l)}^{\prime} \prod_{i=1}^{q-1}\left|k+\tilde{l}_{i}\right|^{p_{i}} Q(k) e^{i k \cdot \Theta \sum_{1}^{q} l_{j}|k|^{-s}} \\
& +\sum_{\substack{\alpha=\left(\alpha_{i}\right)_{i \in I} \in \\
\prod_{i \in I}\{0, \ldots, N\}}}\left(\begin{array}{c}
1 / 2 \\
\alpha
\end{array}\right) \sum_{|k|>U(l)} \frac{1}{|k|^{s+2|\alpha|_{1}-|p|_{1}}} P_{\alpha}(k, l) Q(k) e^{i k \cdot \Theta \sum_{1}^{q} l_{j}} \\
& +G_{N}(l, s),
\end{aligned}
$$

where the map $s \mapsto G_{N}(l, s)$ is a holomorphic function in the half plane $D_{N}:=\left\{\sigma>n+d+|p|_{1}-\frac{N+1}{2}\right\}$ and verifies in it the bound $G_{N}(l, s) \ll_{N, \sigma} 1$ uniformly in $l$.

It follows that

$$
F(l, s)=\sum_{\alpha=\left(\alpha_{i}\right)_{i \in I} \in \prod_{i \in I}\{0, \ldots, N\}^{p_{i}}} H_{\alpha}(l, s)+R_{N}(l, s),
$$

where

$$
\begin{aligned}
H_{\alpha}(l, s):= & \sum_{k \in \mathbb{Z}^{n}}^{\prime}\left(\begin{array}{c}
1 / 2 \\
\alpha
\end{array}\right) \frac{1}{|k|^{s+2|\alpha|_{1}-|p|_{1}}} P_{\alpha}(k, l) Q(k) e^{i k \cdot \Theta \sum_{1}^{q} l_{j}}, \\
R_{N}(l, s):= & \sum_{|k| \leq U(l)}^{\prime} \prod_{i=1}^{q-1}\left|k+\tilde{l}_{i}\right|^{p_{i}} Q(k) e^{i k \cdot \Theta \sum_{1}^{q} l_{j}|k|^{-s}} \\
& -\sum_{|k| \leq U(l)}^{\prime} \sum_{\substack{\alpha=\left(\alpha_{i}\right)_{i \in I} \in \epsilon^{\prime} \\
\prod_{i \in I}\{\ldots, \ldots, N\}^{p_{i}}}}\left(\begin{array}{c}
1 / 2 \\
\alpha
\end{array}\right) \frac{P_{\alpha}(k, l)}{|k|^{s+2|\alpha|_{1}-|p|_{1}}} Q(k) e^{i k \cdot \Theta \sum_{1}^{q} l_{j}}+G_{N}(l, s) .
\end{aligned}
$$


In particular there exists $A(N)>0$ such that $s \mapsto R_{N}(l, s)$ extends holomorphically to the half plane $D_{N}$ and satisfies $R_{N}(l, s) \ll_{N, \sigma} 1+|l|^{A(N)}$ uniformly in $l$.

Write formally

$$
h_{\alpha}(s):=\sum_{l} \tilde{a}_{l} H_{\alpha}(l, s) .
$$

Equation (2.20) and $R_{N}(l, s) \ll_{N, \sigma} 1+|l|^{A(N)}$ imply that

$$
f(s) \sim_{N} \sum_{\alpha=\left(\alpha_{i}\right)_{i \in I} \in \prod_{i \in I}\{0, \ldots, N\}^{p_{i}}} h_{\alpha}(s),
$$

where $\sim_{N}$ means modulo a holomorphic function in $D_{N}$.

Recall the decomposition $\left(\begin{array}{c}1 / 2 \\ \alpha\end{array}\right) P_{\alpha}(k, l) Q(k)=\sum_{\beta \in L(\alpha)} M_{\alpha, \beta}(l) k^{\beta}$. Similarly we decompose $h_{\alpha}(s)=\sum_{\beta \in L(\alpha)} h_{\alpha, \beta}(s)$. Theorem 2.6 now implies that for all $\alpha=\left(\alpha_{i}\right)_{i \in I} \in \prod_{i \in I}\{0, \ldots, N\}^{p_{i}}$ and $\beta \in L(\alpha)$

- the map $s \mapsto h_{\alpha, \beta}(s)$ has a meromorphic continuation to the whole complex plane $\mathbb{C}$ with only one simple possible pole at $s=n+|p|_{1}-2|\alpha|_{1}+d_{\beta}$,

- the residue at this point is equal to

$$
\operatorname{Res}_{s=n+|p|_{1}-2|\alpha|_{1}+d_{\beta}} h_{\alpha, \beta}(s)=\sum_{l \in \mathcal{Z}} \tilde{a}_{l} M_{\alpha, \beta}(l) \int_{u \in S^{n-1}} u^{\beta} d S(u)
$$

where $\left.z:=\left\{l \in(\mathbb{Z})^{n}\right)^{2 q} \mid \sum_{1}^{q} l_{j}=0\right\}$. If the right hand side is zero, $h_{\alpha, \beta}(s)$ is holomorphic in $\mathbb{C}$.

By (2.21), we deduce therefore that $f(s)$ has a meromorphic continuation to the half plane $D_{N}$, with only simple possible poles in the set $\left\{n+|p|_{1}+\left.k|-2 N| p\right|_{1} \leq\right.$ $k \leq d\}$. Taking now $N \rightarrow \infty$ yields the result.

(ii) Let $m \in \mathbb{N}_{0}$ and set $I(m):=\left\{(\alpha, \beta) \in \mathcal{J} \times \mathbb{N}_{0}^{(2 q+1) n} \mid \beta \in L(\alpha)\right.$ and $m=$ $\left.2|\alpha|_{1}-d_{\beta}+d\right\}$. If $(\alpha, \beta) \in I(m)$, then $|\alpha|_{1} \leq m$ and $|\beta|_{1} \leq 3 m+d$, so $I(m)$ is finite. With $N$ chosen such that $2 N|p|_{1}+d>m$, we get by (2.21) and (2.22)

$$
\operatorname{Res}_{s=n+d+|p|_{1}-m} f(s)=\sum_{l \in \mathcal{Z}} \tilde{a}_{l} \sum_{(\alpha, \beta) \in I(m)} M_{\alpha, \beta}(l) \int_{u \in S^{n-1}} u^{\beta} d S(u)=C(f, m)
$$

with the convention $\sum_{\emptyset}=0$. Thus, $n+d+|p|_{1}-m$ is a pole of $f$ if and only if $C(f, m) \neq 0$.

\section{Noncommutative integration on a simple spectral triple}

In this section we revisit the notion of noncommutative integral pioneered by Alain Connes, paying particular attention to the reality (Tomita-Takesaki) operator $J$ and to kernels of perturbed Dirac operators by symmetrized 1-forms. 
3.1. Kernel dimension. We will have to compare here the kernels of $\mathcal{D}$ and $\mathcal{D}_{A}$ which are both finite dimensional:

Lemma 3.1. Let $(\mathcal{A}, \mathcal{H}, \mathcal{D})$ be a spectral triple with a reality operator $J$ and chirality $\chi$. If $A \in \Omega_{\mathcal{D}}^{1}$ is a 1-form, then the fluctuated Dirac operator

$$
\mathcal{D}_{A}:=\mathcal{D}+A+\epsilon J A J^{-1}
$$

(where $\mathcal{D} J=\epsilon J \mathcal{D}, \epsilon= \pm 1$ ) is an operator with compact resolvent, and in particular its kernel $\operatorname{Ker} \mathcal{D}_{A}$ is a finite dimensional space. This space is invariant by $J$ and $\chi$.

Proof. Let $T$ be a bounded operator and let $z, z^{\prime}$ be in the resolvent of $\mathcal{D}+T$ and $\mathcal{D}$, respectively. Then

$$
(\mathcal{D}+T-z)^{-1}=\left(\mathcal{D}-z^{\prime}\right)^{-1}\left[1-\left(T+z^{\prime}-z\right)(\mathcal{D}+T-z)^{-1}\right] .
$$

Since $\left(\mathcal{D}-z^{\prime}\right)^{-1}$ is compact by hypothesis and since the term in bracket is bounded, $\mathcal{D}+T$ has a compact resolvent. Applying this to $T=A+\epsilon J A J^{-1}, \mathcal{D}_{A}$ has a finite dimensional kernel (see for instance [27], Theorem 6.29).

Since according to the dimension, $J^{2}= \pm 1, J$ commutes or anticommutes with $\chi, \chi$ commutes with the elements in the algebra $\mathcal{A}$ and $\mathcal{D} \chi=-\chi \mathcal{D}$ (see [9] or [23], p. 405), we obtain that $\mathcal{D}_{A} \chi=-\chi \mathcal{D}_{A}$ and $\mathcal{D}_{A} J= \pm J \mathcal{D}_{A}$, which gives the result.

3.2. Pseudodifferential operators. Let $(A, \mathcal{D}, \mathcal{H})$ be a given real regular spectral triple of dimension $n$. Let $P_{0}$ be the projection on $\operatorname{Ker} \mathcal{D}$ and let $P_{A}$ be the projection on Ker $\mathcal{D}_{A}$. Write $D:=\mathcal{D}+P_{0}, D_{A}:=\mathcal{D}_{A}+P_{A}$. The maps $P_{0}$ and $P_{A}$ are thus finite-rank selfadjoint bounded operators. We remark that $D$ and $D_{A}$ are selfadjoint invertible operators with compact inverses.

Remark 3.2. Since we only need to compute the residues and the value at 0 of the $\zeta_{D}, \zeta_{D_{A}}$ functions, it is not necessary to define the operators $\mathcal{D}^{-1}$ or $\mathcal{D}_{A}^{-1}$ and the associated zeta functions. However, we note that all the work presented here could be done using the process of Higson in [26] which proves that we can add any smoothing operator to $\mathcal{D}$ or $\mathcal{D}_{A}$ such that the result is invertible without changing anything in the computation of residues.

Define for any $\alpha \in \mathbb{R}$,

$$
\begin{aligned}
& O P^{0}:=\left\{T \mid t \mapsto F_{t}(T) \in C^{\infty}(\mathbb{R}, \mathcal{B}(\mathcal{H}))\right\}, \\
& O P^{\alpha}:=\left\{\left.T|T| D\right|^{-\alpha} \in O P^{0}\right\},
\end{aligned}
$$


where $F_{t}(T):=e^{i t|D|} T e^{-i t|D|}=e^{i t|\mathcal{D}|} T e^{-i t|\mathcal{D}|}$ since $|D|=|\mathcal{D}|+P_{0}$. Define

$$
\begin{aligned}
\delta(T) & :=[|D|, T], \\
\nabla(T) & :=\left[\mathcal{D}^{2}, T\right], \\
\sigma_{s}(T) & :=|D|^{s} T|D|^{-s}, \quad s \in \mathbb{C} .
\end{aligned}
$$

It has been shown in [13] that $O P^{0}=\bigcap_{p \geq 0} \operatorname{Dom}\left(\delta^{p}\right)$. In particular, $O P^{0}$ is a subalgebra of $\mathcal{B}(\mathcal{H})$ (while elements of $O P^{\alpha}$ are not necessarily bounded for $\alpha>0$ ) and $\mathcal{A} \subseteq O P^{0}, J \mathcal{A} J^{-1} \subseteq O P^{0},[\mathcal{D}, \mathcal{A}] \subseteq O P^{0}$. Note that $P_{0} \in O P^{-\infty}$ and $\delta\left(O P^{0}\right) \subseteq O P^{0}$.

For any $t>0, \mathcal{D}^{t}$ and $|\mathcal{D}|^{t}$ are in $O P^{t}$, and for any $\alpha \in \mathbb{R}, D^{\alpha}$ and $|D|^{\alpha}$ are in $O P^{\alpha}$. By hypothesis, $|D|^{-n} \in \mathcal{L}^{(1, \infty)}(\mathcal{H})$, so $O P^{-\alpha} \subseteq \mathcal{L}^{1}(\mathcal{H})$ for any $\alpha>n$.

Lemma 3.3 ([13]). (i) For any $T \in O P^{0}$ and $s \in \mathbb{C}, \sigma_{s}(T) \in O P^{0}$.

(ii) For any $\alpha, \beta \in \mathbb{R}, O P^{\alpha} O P^{\beta} \subseteq O P^{\alpha+\beta}$.

(iii) If $\alpha \leq \beta, O P^{\alpha} \subseteq O P^{\beta}$.

(iv) For any $\alpha, \delta\left(O P^{\alpha}\right) \subseteq O P^{\alpha}$.

(v) For any $\alpha$ and $T \in O P^{\alpha}, \nabla(T) \in O P^{\alpha+1}$.

Proof. See the appendix.

Remark 3.4. Any operator in $O P^{\alpha}, \alpha \in \mathbb{R}$, extends as a continuous linear operator from Dom $|D|^{\alpha+1}$ to Dom $|D|$, where the Dom $|D|^{\alpha}$ spaces have their natural norms; see [13], [26].

We now introduce a definition of pseudodifferential operators in a slightly different way than in [13], [8], [26], which in particular pays attention to the reality operator $J$ and the kernel of $\mathcal{D}$ and allows $\mathcal{D}$ and $|D|^{-1}$ to be pseudodifferential operators. It is more in the spirit of [11].

Definition 3.5. Let us define $\mathcal{D}(\mathcal{A})$ as the polynomial algebra generated by $\mathcal{A}$, $J \mathcal{A} J^{-1}, \mathcal{D}$ and $|\mathcal{D}|$. A pseudodifferential operator is an operator $T$ so that there is an element $d \in \mathbb{Z}$ such that for any $N \in \mathbb{N}$, there exist $p \in \mathbb{N}_{0}, P \in \mathcal{D}(\mathcal{A})$ and $R \in O P^{-N}$ ( $p, P$ and $R$ may depend on $N$ ) with $P D^{-2 p} \in O P^{d}$ and

$$
T=P D^{-2 p}+R \text {. }
$$

Define $\Psi(\mathcal{A})$ as the set of pseudodifferential operators and $\Psi(\mathcal{A})^{k}:=\Psi(\mathcal{A}) \cap O P^{k}$.

Note that if $A$ is a 1 -form, then $A$ and $J A J^{-1}$ are in $\mathcal{D}(\mathcal{A})$ and, moreover, it follows that $\mathcal{D}(\mathcal{A}) \subseteq \bigcup_{p \in \mathbb{N}_{0}} O P^{p}$. Since $|\mathcal{D}| \in \mathcal{D}(\mathcal{A})$ by construction and $P_{0}$ is a pseudodifferential operator, $|D|^{p}$ is a pseudodifferential operator (in $O P^{p}$ ) for any $p \in \mathbb{Z}$. We also remark that $\mathcal{D}(\mathcal{A}) \subseteq \Psi(\mathcal{A}) \subseteq \bigcup_{k \in \mathbb{Z}} O P^{k}$. 
Lemma 3.6 ([13], [8]). The set of all pseudodifferential operators $\Psi(\mathcal{A})$ is an algebra. Moreover, if $T \in \Psi(\mathcal{A})^{d}$ and $T \in \Psi(\mathcal{A})^{d^{\prime}}$, then $T T^{\prime} \in \Psi(\mathcal{A})^{d+d^{\prime}}$.

Proof. See the appendix.

Due to the little difference of behavior between scalar and nonscalar pseudodifferential operators (i.e., when coefficients like $[\mathcal{D}, a], a \in \mathcal{A}$, appear in $P$ of Definition 3.5), it is convenient to introduce the following notation.

Definition 3.7. Let $\mathcal{D}_{1}(\mathcal{A})$ be the algebra generated by $\mathcal{A}, J \mathcal{A} J^{-1}$ and $\mathcal{D}$, and let $\Psi_{1}(\mathcal{A})$ be the set of pseudodifferential operators constructed as before, with $\mathcal{D}_{1}(\mathcal{A})$ instead of $\mathcal{D}(\mathcal{A})$. Note that $\Psi_{1}(\mathcal{A})$ is subalgebra of $\Psi(\mathcal{A})$.

Observe that $\Psi_{1}(\mathcal{A})$ does not necessarily contain operators such as $|D|^{k}$ where $k \in \mathbb{Z}$ is odd. This algebra is similar to the one defined in [11].

3.3. Zeta functions and dimension spectrum. For any operator $B$ and if $X$ is either $D$ or $D_{A}$, we define

$$
\begin{aligned}
& \zeta_{X}^{B}(s):=\operatorname{Tr}\left(B|X|^{-s}\right), \\
& \zeta_{X}(s):=\operatorname{Tr}\left(|X|^{-s}\right) .
\end{aligned}
$$

The dimension spectrum $\operatorname{Sd}(\mathcal{A}, \mathcal{H}, \mathcal{D})$ of a spectral triple has been defined in [8], [13]. It is extended here to pay attention to the operator $J$ and to our definition of pseudodifferential operator.

Definition 3.8. The spectrum dimension of the $\operatorname{spectral}$ triple is the $\operatorname{subset} \operatorname{Sd}(\mathcal{A}, \mathcal{H}, \mathcal{D})$ of all poles of the functions $\zeta_{D}^{P}:=s \mapsto \operatorname{Tr}\left(P|D|^{-s}\right)$ where $P$ is any pseudodifferential operator in $O P^{0}$. The spectral triple $(\mathcal{A}, \mathcal{H}, \mathcal{D})$ is simple when these poles are all simple.

Remark 3.9. If $\operatorname{Sp}(\mathcal{A}, \mathcal{H}, \mathcal{D})$ denotes the set of all poles of the functions $s \mapsto$ $\operatorname{Tr}\left(P|D|^{-s}\right)$, where $P$ is any pseudodifferential operator, then $\operatorname{Sd}(\mathcal{A}, \mathcal{H}, \mathcal{D}) \subseteq$ $\operatorname{Sp}(\mathcal{A}, \mathcal{H}, \mathcal{D})$.

If $\operatorname{Sp}(\mathcal{A}, \mathcal{H}, \mathcal{D})=\mathbb{Z}$, then $\operatorname{Sd}(\mathcal{A}, \mathcal{H}, \mathcal{D})=\left\{n-k \mid k \in \mathbb{N}_{0}\right\}:$ indeed, if $P$ is a pseudodifferential operator in $O P^{0}$, and $q \in \mathbb{N}$ is such that $q>n$, then $P|D|^{-s}$ is in $O P^{-\operatorname{Re}(s)}$, so is trace-class for $s$ in a neighborhood of $q$; hence $q$ cannot be a pole of $s \mapsto \operatorname{Tr}\left(P|D|^{-s}\right)$.

Remark 3.10. The set $\operatorname{Sp}(\mathcal{A}, \mathcal{H}, \mathcal{D})$ is also the set of all poles of functions $s \mapsto$ $\operatorname{Tr}\left(B|D|^{-s-2 p}\right)$ where $p \in \mathbb{N}_{0}$ and $B \in \mathcal{D}(\mathcal{A})$. 
3.4. The noncommutative integral $f$. We already defined the one parameter group $\sigma_{z}(T):=|D|^{z} T|D|^{-z}, z \in \mathbb{C}$.

Introducing the notation (recall that $\nabla(T)=\left[\mathcal{D}^{2}, T\right]$ ) for an operator $T$,

$$
\varepsilon(T):=\nabla(T) D^{-2},
$$

we get from [11], (2.44), the following expansion for $T \in O P^{q}$ :

$$
\sigma_{z}(T) \sim \sum_{r=0}^{N} g(z, r) \varepsilon^{r}(T) \bmod O P^{-N-1+q},
$$

where $g(z, r):=\frac{1}{r !}\left(\frac{z}{2}\right) \ldots\left(\frac{z}{2}-(r-1)\right)=\left(\begin{array}{c}z / 2 \\ r\end{array}\right)$ with the convention $g(z, 0):=1$.

We define the noncommutative integral by

$$
f T:=\operatorname{Res}_{s=0} \zeta_{D}^{T}(s)=\operatorname{Res}_{s=0} \operatorname{Tr}\left(T|D|^{-s}\right)
$$

Proposition 3.11 ([13]). If the spectral triple is simple, then $f$ is a trace on $\Psi(\mathcal{A})$.

Proof. See the appendix.

\section{Residues of $\zeta_{D_{A}}$ for a spectral triple with simple dimension spectrum}

We fix a regular spectral triple $(\mathcal{A}, \mathcal{H}, \mathcal{D})$ of dimension $n$ and a selfadjoint 1 -form $A$.

Recall that

$$
\begin{aligned}
& \mathcal{D}_{A}:=\mathcal{D}+\tilde{A}, \\
& D_{A}:=\mathcal{D}_{A}+P_{A},
\end{aligned}
$$

where $\tilde{A}:=A+\varepsilon J A J^{-1}$ and $P_{A}$ is the projection on $\operatorname{Ker} \mathcal{D}_{A}$. Observe that $\tilde{A} \in$ $\mathcal{D}(\mathcal{A}) \cap O P^{0}$ and $\mathcal{D}_{A} \in \mathcal{D}(\mathcal{A}) \cap O P^{1}$.

Write

$$
V_{A}:=P_{A}-P_{0}
$$

As the following lemma shows, $V_{A}$ is a smoothing operator:

Lemma 4.1. (i) $\bigcap_{k \geq 1} \operatorname{Dom}\left(\mathcal{D}_{A}\right)^{k} \subseteq \bigcap_{k \geq 1} \operatorname{Dom}|D|^{k}$.

(ii) $\operatorname{Ker} \mathcal{D}_{A} \subseteq \bigcap_{k \geq 1} \operatorname{Dom}|D|^{k}$.

(iii) For any $\alpha, \beta \in \mathbb{R},|D|^{\beta} P_{A}|D|^{\alpha}$ is bounded.

(iv) $P_{A} \in O P^{-\infty}$. 
Proof. (i) For any $p \in \mathbb{N}$ let us define $R_{p}:=\left(\mathcal{D}_{A}\right)^{p}-\mathcal{D}^{p}$, so $R_{p} \in O P^{p-1}$ and $R_{p}\left(\operatorname{Dom}|D|^{p}\right) \subseteq \operatorname{Dom}|D|$ (see Remark 3.4).

Fix $k \in \mathbb{N}, k \geq 2$. Since $\operatorname{Dom} \mathcal{D}_{A}=\operatorname{Dom} \mathcal{D}=\operatorname{Dom}|D|$, we have

$$
\operatorname{Dom}\left(\mathcal{D}_{A}\right)^{k}=\left\{\phi \in \operatorname{Dom}|D|\left|\left(\mathcal{D}^{j}+R_{j}\right) \phi \in \operatorname{Dom}\right| D \mid \text { for all } 1 \leq j \leq k-1\right\} .
$$

Let $\phi \in \operatorname{Dom}\left(\mathcal{D}_{A}\right)^{k}$. We prove by recurrence that $\phi \in \operatorname{Dom}|D|^{j+1}$ for any $j \in$ $\{1, \ldots, k-1\}$. We have $\phi \in \operatorname{Dom}|D|$ and $\left(\mathcal{D}+R_{1}\right) \phi \in \operatorname{Dom}|D|$. Thus, since $R_{1} \phi \in \operatorname{Dom}|D|$, it follows that $\mathcal{D} \phi \in \operatorname{Dom}|D|$, which proves that $\phi \in \operatorname{Dom}|D|^{2}$. Hence the case $j=1$ is done.

Suppose now that $\phi \in \operatorname{Dom}|D|^{j+1}$ for a $j \in\{1, \ldots, k-2\}$. Since $\left(\mathcal{D}^{j+1}+\right.$ $\left.R_{j+1}\right) \phi \in \operatorname{Dom}|D|$, and $R_{j+1} \phi \in \operatorname{Dom}|D|$, we get $\mathcal{D}^{j+1} \phi \in \operatorname{Dom}|D|$, which proves that $\phi \in \operatorname{Dom}|D|^{j+2}$.

Finally, if we set $j=k-1$, we get $\phi \in \operatorname{Dom}|D|^{k}$, so $\operatorname{Dom}\left(\mathcal{D}_{A}\right)^{k} \subseteq \operatorname{Dom}|D|^{k}$.

(ii) follows from $\operatorname{Ker} \mathcal{D}_{A} \subseteq \bigcap_{k \geq 1} \operatorname{Dom}\left(\mathcal{D}_{A}\right)^{k}$ and (i).

(iii) Let us first check that $|D|^{\alpha} \bar{P}_{A}$ is bounded. We define $D_{0}$ as the operator with domain Dom $D_{0}=\operatorname{Im} P_{A} \cap \operatorname{Dom}|D|^{\alpha}$ and such that $D_{0} \phi=|D|^{\alpha} \phi$. Since Dom $D_{0}$ is finite dimensional, $D_{0}$ extends to a bounded operator on $\mathcal{H}$ with finite rank. We have

$$
\sup _{\substack{\phi \in \operatorname{Dom}|D|^{\alpha} P_{A} \\\|\phi\| \leq 1}}\left\||D|^{\alpha} P_{A} \phi\right\| \leq \sup _{\substack{\phi \in \operatorname{Dom} D_{0} \\\|\phi\| \leq 1}}\left\||D|^{\alpha} \phi\right\|=\left\|D_{0}\right\|<\infty,
$$

so $|D|{ }^{\alpha} P_{A}$ is bounded. Note that by (ii), Dom $D_{0}=\operatorname{Im} P_{A}$ and $\operatorname{Dom}|D|{ }^{\alpha} P_{A}=\mathcal{H}$. We now show that $P_{A}|D|^{\alpha}$ is bounded: Let $\phi \in \operatorname{Dom} P_{A}|D|^{\alpha}=\operatorname{Dom}|D|^{\alpha}$. By (ii), we have $\operatorname{Im} P_{A} \subseteq \operatorname{Dom}|D|^{\alpha}$, so we get

$$
\begin{aligned}
\left\|P_{A}|D|^{\alpha} \phi\right\| & \leq \sup _{\substack{\psi \in \operatorname{Im} P_{A} \\
\|\psi\| \leq 1}}\left|\left\langle\psi,|D|^{\alpha} \phi\right\rangle\right| \leq \sup _{\substack{\psi \in \operatorname{Im} P_{A} \\
\|\psi\| \leq 1}}\left|\left\langle|D|^{\alpha} \psi, \phi\right\rangle\right| \\
& \leq \sup _{\substack{\psi \in \operatorname{Im} P_{A} \\
\|\psi\| \leq 1}}\left\||D|^{\alpha} \psi\right\|\|\phi\|=\left\|D_{0}\right\|\|\phi\| .
\end{aligned}
$$

(iv) For any $k \in \mathbb{N}_{0}$ and $t \in \mathbb{R}, \delta^{k}\left(P_{A}\right)|D|^{t}$ is a linear combination of terms of the form $|D|^{\beta} P_{A}|D|^{\alpha}$, so the result follows from (iii).

Remark 4.2. We will see later in the noncommutative torus example how important the difference between $\mathcal{D}_{A}$ and $\mathcal{D}+A$ is. In particular, the inclusion $\operatorname{Ker} \mathcal{D} \subseteq$ $\operatorname{Ker} \mathcal{D}+A$ is not satisfied since $A$, in contrast to $\tilde{A}$, does not preserve $\operatorname{Ker} \mathcal{D}$.

The coefficient of the nonconstant term $\Lambda^{k}(k>0)$ in the expansion (1.5) of the spectral action $S\left(\mathcal{D}_{A}, \Phi, \Lambda\right)$ is equal to the residue of $\zeta_{D_{A}}(s)$ at $k$. We will see in this section how we can compute these residues in terms of the noncommutative integral of certain operators. 
Define for any operator $T, p \in \mathbb{N}, s \in \mathbb{C}$,

$$
K_{p}(T, s):=(-s / 2)^{p} \int_{0 \leq t_{1} \leq \cdots \leq t_{p} \leq 1} \sigma_{-s t_{1}}(T) \ldots \sigma_{-s t_{p}}(T) d t
$$

with $d t:=d t_{1} \ldots d t_{p}$.

Note that if $T \in O P^{\alpha}$, then $\sigma_{z}(T) \in O P^{\alpha}$ for $z \in \mathbb{C}$ and $K_{p}(T, s) \in O P^{\alpha p}$.

Let us define

$$
\begin{aligned}
X & :=\mathcal{D}_{A}^{2}-\mathcal{D}^{2}=\tilde{A} \mathcal{D}+\mathcal{D} \tilde{A}+\tilde{A}^{2}, \\
X_{V} & :=X+V_{A}
\end{aligned}
$$

then $X \in \mathcal{D}_{1}(\mathcal{A}) \cap O P^{1}$ and by Lemma 4.1,

$$
X_{V} \sim X \bmod O P^{-\infty}
$$

We will use

$$
Y:=\log \left(D_{A}^{2}\right)-\log \left(D^{2}\right),
$$

which makes sense since $D_{A}^{2}=\mathcal{D}_{A}^{2}+P_{A}$ is invertible for any $A$.

By definition of $X_{V}$, we see that

$$
Y=\log \left(D^{2}+X_{V}\right)-\log \left(D^{2}\right)
$$

Lemma 4.3 ([11]). (i) The map $Y$ is a pseudodifferential operator in $O P^{-1}$ with the following expansion for any $N \in \mathbb{N}$ :

$$
Y \sim \sum_{p=1}^{N} \sum_{k_{1}, \ldots, k_{p}=0}^{N-p} \frac{(-1)^{|k|_{1}+p+1}}{|k|_{1}+p} \nabla^{k_{p}}\left(X \nabla^{k_{p-1}}\left(\ldots X \nabla^{k_{1}}(X) \ldots\right)\right) D^{-2\left(|k|_{1}+p\right)}
$$

$\bmod O P^{-N-1}$.

(ii) For any $N \in \mathbb{N}$ and $s \in \mathbb{C}$ we have

$$
\left|D_{A}\right|^{-s} \sim|D|^{-s}+\sum_{p=1}^{N} K_{p}(Y, s)|D|^{-s} \bmod O P^{-N-1-\operatorname{Re}(s)} .
$$

Proof. (i) We follow [11], Lemma 2.2. By functional calculus, $Y=\int_{0}^{\infty} I(\lambda) d \lambda$ where

$$
I(\lambda) \sim \sum_{p=1}^{N}(-1)^{p+1}\left(\left(D^{2}+\lambda\right)^{-1} X_{V}\right)^{p}\left(D^{2}+\lambda\right)^{-1} \bmod O P^{-N-3}
$$


By (4.1), $\left(\left(D^{2}+\lambda\right)^{-1} X_{V}\right)^{p} \sim\left(\left(D^{2}+\lambda\right)^{-1} X\right)^{p} \bmod O P^{-\infty}$ and we get

$$
I(\lambda) \sim \sum_{p=1}^{N}(-1)^{p+1}\left(\left(D^{2}+\lambda\right)^{-1} X\right)^{p}\left(D^{2}+\lambda\right)^{-1} \bmod O P^{-N-3} .
$$

We set $A_{p}(X):=\left(\left(D^{2}+\lambda\right)^{-1} X\right)^{p}\left(D^{2}+\lambda\right)^{-1}$ and $L:=\left(D^{2}+\lambda\right)^{-1} \in O P^{-2}$ for a fixed $\lambda$. Since $\left[D^{2}+\lambda, X\right] \sim \nabla(X) \bmod O P^{-\infty}$, a recurrence proves that if $T$ is an operator in $O P^{r}$, then for $q \in \mathbb{N}_{0}$,

$$
A_{1}(T)=L T L \sim \sum_{k=0}^{q}(-1)^{k} \nabla^{k}(T) L^{k+2} \bmod O P^{r-q-5} .
$$

With $A_{p}(X)=L X A_{p-1}(X)$ another recurrence gives for any $q \in \mathbb{N}_{0}$,

$$
A_{p}(X) \sim \sum_{k_{1}, \ldots, k_{p}=0}^{q}(-1)^{|k|_{1}} \nabla^{k_{p}}\left(X \nabla^{k_{p-1}}\left(\ldots X \nabla^{k_{1}}(X) \ldots\right)\right) L^{|k|_{1}+p+1}
$$

$\bmod O P^{-q-p-3}$, which implies that

$$
I(\lambda) \sim \sum_{p=1}^{N}(-1)^{p+1} \sum_{k_{1}, \ldots, k_{p}=0}^{N-p}(-1)^{|k|_{1}} \nabla^{k_{p}}\left(X \nabla^{k_{p-1}}\left(\ldots X \nabla^{k_{1}}(X) \ldots\right)\right) L^{|k|_{1}+p+1}
$$

$\bmod O P^{-N-3}$. With $\int_{0}^{\infty}\left(D^{2}+\lambda\right)^{-\left(|k|_{1}+p+1\right)} d \lambda=\frac{1}{|k|_{1}+p} D^{-2\left(|k|_{1}+p\right)}$ we get the result provided we control the remainders. Such a control is given in [11], (2.27).

(ii) We have $\left|D_{A}\right|^{-s}=e^{B-(s / 2) Y} e^{-B}|D|^{-s}$ where $B:=(-s / 2) \log \left(D^{2}\right)$. Following [11], Theorem 2.4, we obtain that

$$
\left|D_{A}\right|^{-s}=|D|^{-s}+\sum_{p=1}^{\infty} K_{p}(Y, s)|D|^{-s}
$$

and each $K_{p}(Y, s)$ is in $O P^{-p}$.

Corollary 4.4. For any $p \in \mathbb{N}$ and $r_{1}, \ldots, r_{p} \in \mathbb{N}_{0}$ we have $\varepsilon^{r_{1}}(Y) \ldots \varepsilon^{r_{p}}(Y) \in$ $\Psi_{1}(\mathcal{A})$.

Proof. If for any $q \in \mathbb{N}$ and $k=\left(k_{1}, \ldots, k_{q}\right) \in \mathbb{N}_{0}^{q}$,

$$
\Gamma_{q}^{k}(X):=\frac{(-1)^{|k|_{1}+q+1}}{|k|_{1}+q} \nabla^{k_{q}}\left(X \nabla^{k_{q-1}}\left(\ldots X \nabla^{k_{1}}(X) \ldots\right)\right),
$$

then $\Gamma_{q}^{k}(X) \in O P^{|k|_{1}+q}$. For any $N \in \mathbb{N}$,

$$
Y \sim \sum_{q=1}^{N} \sum_{k_{1}, \ldots, k_{q}=0}^{N-q} \Gamma_{q}^{k}(X) D^{-2\left(|k|_{1}+q\right)} \bmod O P^{-N-1}
$$


Note that the $\Gamma_{q}^{k}(X)$ are in $\mathcal{D}_{1}(\mathcal{A})$, which in view of (4.4) shows that $Y$ and thus $\varepsilon^{r}(Y)=\nabla^{r}(Y) D^{-2 r}$ are also in $\Psi_{1}(\mathcal{A})$.

We remark, as in [10], that the fluctuations leave invariant the first term of the spectral action (1.5). This is a generalization of the fact that in the commutative case, the noncommutative integral depends only on the principal symbol of the Dirac operator $\mathcal{D}$ and this symbol is stable by adding a gauge potential like in $\mathcal{D}+A$. Note however that the symmetrized gauge potential $A+\epsilon J A J^{-1}$ is always zero in this case for any selfadjoint 1 -form $A$.

Lemma 4.5. If the spectral triple is simple, then formula (1.6) can be extended as

$$
\zeta_{D_{A}}(0)-\zeta_{D}(0)=\sum_{q=1}^{n} \frac{(-1)^{q}}{q} f\left(\tilde{A} D^{-1}\right)^{q}
$$

Proof. Since the spectral triple is simple, equation (4.3) entails that

$$
\zeta_{D_{A}}(0)-\zeta_{D}(0)=\operatorname{Tr}\left(K_{1}(Y, s)|D|^{-s}\right)_{\mid s=0} .
$$

Thus, with (3.1), we get $\zeta_{D_{A}}(0)-\zeta_{D}(0)=-\frac{1}{2} f Y$. Replacing $A$ by $\tilde{A}$, the same proof as in [11] gives

$$
-\frac{1}{2} f Y=\sum_{q=1}^{n} \frac{(-1)^{q}}{q} f\left(\tilde{A} D^{-1}\right)^{q}
$$

Lemma 4.6. For any $k \in \mathbb{N}_{0}$ we have

$$
\begin{aligned}
& \operatorname{Res}_{s=n-k} \zeta_{D_{A}}(s) \\
& \quad=\operatorname{Res}_{s=n-k} \zeta_{D}(s)+\sum_{p=1}^{k} \sum_{r_{1}, \ldots, r_{p}=0}^{k-p} \operatorname{Res}_{s=n-k} h(s, r, p) \operatorname{Tr}\left(\varepsilon^{r_{1}}(Y) \ldots \varepsilon^{r_{p}}(Y)|D|^{-s}\right),
\end{aligned}
$$

where

$$
h(s, r, p):=(-s / 2)^{p} \int_{0 \leq t_{1} \leq \cdots \leq t_{p} \leq 1} g\left(-s t_{1}, r_{1}\right) \ldots g\left(-s t_{p}, r_{p}\right) d t .
$$

Proof. From Lemma 4.3 (ii) it follows that $\left|D_{A}\right|^{-s} \sim|D|^{-s}+\sum_{p=1}^{k} K_{p}(Y, s)|D|^{-s}$ $\bmod O P^{-(k+1)-\operatorname{Re}(s)}$, where the convention $\sum_{\emptyset}=0$ is used. Thus, we get for $s$ in a neighborhood of $n-k$,

$$
\left|D_{A}\right|^{-s}-|D|^{-s}-\sum_{p=1}^{k} K_{p}(Y, s)|D|^{-s} \in O P^{-(k+1)-\operatorname{Re}(s)} \subseteq \mathcal{L}^{1}(\mathcal{H}),
$$


which gives

$$
\operatorname{Res}_{s=n-k} \zeta_{D_{A}}(s)=\operatorname{Res}_{s=n-k} \zeta_{D}(s)+\sum_{p=1}^{k} \operatorname{Res}_{s=n-k} \operatorname{Tr}\left(K_{p}(Y, s)|D|^{-s}\right)
$$

Let us fix $1 \leq p \leq k$ and $N \in \mathbb{N}$. By (3.1) we get

$$
\begin{gathered}
K_{p}(Y, s) \sim(-s / 2)^{p} \int_{0 \leq t_{1} \leq \ldots t_{p} \leq 1} \sum_{r_{1}, \ldots, r_{p}=0}^{N} g\left(-s t_{1}, r_{1}\right) \ldots g\left(-s t_{p}, r_{p}\right) \\
\varepsilon^{r_{1}}(Y) \ldots \varepsilon^{r_{p}}(Y) d t \bmod O P^{-N-p-1} .
\end{gathered}
$$

If we now take $N=k-p$, we obtain that

$K_{p}(Y, s)|D|^{-s}-\sum_{r_{1}, \ldots, r_{p}=0}^{k-p} h(s, r, p) \varepsilon^{r_{1}}(Y) \ldots \varepsilon^{r_{p}}(Y)|D|^{-s} \in O P^{-k-1-\operatorname{Re}(s)} \subseteq \mathcal{L}^{1}(\mathcal{H})$

for $s$ in a neighborhood of $n-k$, so (4.6) gives the result.

Our operators $\left|D_{A}\right|^{k}$ are pseudodifferential operators:

Lemma 4.7. For any $k \in \mathbb{Z}$ we have $\left|D_{A}\right|^{k} \in \Psi^{k}(\mathcal{A})$.

Proof. Using (4.7), we see that $K_{p}(Y, s)$ is a pseudodifferential operator in $O P^{-p}$, so (4.2) shows that $\left|D_{A}\right|^{k}$ is a pseudodifferential operator in $O P^{k}$.

The following result is quite important since it shows that one can use $f$ for $D$ or $D_{A}$ :

Proposition 4.8. If the spectral triple is simple, then $\underset{s=0}{\operatorname{Res}} \operatorname{Tr}\left(P\left|D_{A}\right|^{-s}\right)=f P$ for any pseudodifferential operator $P$. In particular, for any $k \in \mathbb{N}_{0}$,

$$
f\left|D_{A}\right|^{-(n-k)}=\operatorname{Res}_{s=n-k} \zeta_{D_{A}}(s) \text {. }
$$

Proof. Suppose that $P \in O P^{k}$ with $k \in \mathbb{Z}$ and fix $p \geq 1$. With (4.7) we see that

$$
\begin{aligned}
& P K_{p}(Y, s)|D|^{-s} \\
& \quad \sim \sum_{r_{1}, \ldots, r_{p}=0}^{N} h(s, r, p) P \varepsilon^{r_{1}}(Y) \ldots \varepsilon^{r_{p}}(Y)|D|^{-s} \bmod O P^{-N-p-1+k-\operatorname{Re}(s)}
\end{aligned}
$$


for any $N \in \mathbb{N}$. Thus if we take $N=n-p+k$, we get

$$
\operatorname{Res}_{s=0} \operatorname{Tr}\left(P K_{p}(Y, s)|D|^{-s}\right)=\sum_{r_{1}, \ldots, r_{p}=0}^{n-p+k} \operatorname{Res}_{s=0} h(s, r, p) \operatorname{Tr}\left(P \varepsilon^{r_{1}}(Y) \ldots \varepsilon^{r_{p}}(Y)|D|^{-s}\right) \text {. }
$$

Now $s=0$ is a zero of the analytic function $s \mapsto h(s, r, p)$. Moreover, the map $s \mapsto \operatorname{Tr} P \varepsilon^{r_{1}}(Y) \ldots \varepsilon^{r_{p}}(Y)|D|^{-s}$ has only simple poles. So, by hypothesis, we see that $\operatorname{Res}_{s=0} h(s, r, p) \operatorname{Tr}\left(P \varepsilon^{r_{1}}(Y) \ldots \varepsilon^{r_{p}}(Y)|D|^{-s}\right)=0$ and

$$
\underset{s=0}{\operatorname{Res}} \operatorname{Tr}\left(P K_{p}(Y, s)|D|^{-s}\right)=0 .
$$

Using (4.2), $P\left|D_{A}\right|^{-s} \sim P|D|^{-s}+\sum_{p=1}^{k+n} P K_{p}(Y, s)|D|^{-s} \bmod O P^{-n-1-\operatorname{Re}(s)}$ and thus

$$
\underset{s=0}{\operatorname{Res}} \operatorname{Tr}\left(P\left|D_{A}\right|^{-s}\right)=f P+\sum_{p=1}^{k+n} \operatorname{Res}_{s=0} \operatorname{Tr}\left(P K_{p}(Y, s)|D|^{-s}\right) .
$$

The result now follows from (4.8) and (4.9). To obtain the last equality, one uses the pseudodifferential operator $\left|D_{A}\right|^{-(n-k)}$.

Proposition 4.9. If the spectral triple is simple, then

$$
f\left|D_{A}\right|^{-n}=f|D|^{-n}
$$

Proof. This follows from Lemma 4.6 and the previous proposition for $k=0$.

Lemma 4.10. If the spectral triple is simple, then
(i) $f\left|D_{A}\right|^{-(n-1)}=f|D|^{-(n-1)}-\left(\frac{n-1}{2}\right) f X|D|^{-n-1}$,
(ii) $f\left|D_{A}\right|^{-(n-2)}=f|D|^{-(n-2)}+\frac{n-2}{2}\left(-f X|D|^{-n}+\frac{n}{4} f X^{2}|D|^{-2-n}\right)$.

Proof. (i) By (4.2),

$$
\begin{aligned}
\operatorname{Res}_{s=n-1} \zeta_{D_{A}}(s)-\zeta_{D}(s) & =\operatorname{Res}_{s=n-1}(-s / 2) \operatorname{Tr}\left(Y|D|^{-s}\right) \\
& =-\frac{n-1}{2} \operatorname{Res}_{s=0} \operatorname{Tr}\left(Y|D|^{-(n-1)}|D|^{-s}\right)
\end{aligned}
$$

where for the last equality we use the simple dimension spectrum hypothesis. Lemma 4.3 (i) yields $Y \sim X D^{-2} \bmod O P^{-2}$ and $Y|D|^{-(n-1)} \sim X|D|^{-n-1} \bmod$ $O P^{-n-1} \subseteq \mathcal{L}^{1}(\mathcal{H})$. Thus,

$$
\operatorname{Res}_{s=0} \operatorname{Tr}\left(Y|D|^{-(n-1)}|D|^{-s}\right)=\operatorname{Res}_{s=0} \operatorname{Tr}\left(X|D|^{-n-1}|D|^{-s}\right)=f X|D|^{-n-1} .
$$


(ii) Lemma 4.6 (ii) gives

$$
\begin{aligned}
\underset{s=n-2}{\operatorname{Res}} \zeta_{D_{A}}(s)= & \operatorname{Res}_{s=n-2} \zeta_{D}(s)+\operatorname{Res}_{s=n-2} \sum_{r=0}^{1} h(s, r, 1) \operatorname{Tr}\left(\varepsilon^{r}(Y)|D|^{-s}\right) \\
& +h(s, 0,2) \operatorname{Tr}\left(Y^{2}|D|^{-s}\right)
\end{aligned}
$$

We have $h(s, 0,1)=-\frac{s}{2}, h(s, 1,1)=\frac{1}{2}\left(\frac{s}{2}\right)^{2}$ and $h(s, 0,2)=\frac{1}{2}\left(\frac{s}{2}\right)^{2}$. Using again Lemma 4.3 (i), we obtain that

$$
Y \sim X D^{-2}-\frac{1}{2} \nabla(X) D^{-4}-\frac{1}{2} X^{2} D^{-4} \bmod O P^{-3} .
$$

Thus,

$$
\operatorname{Res}_{s=n-2} \operatorname{Tr}\left(Y|D|^{-s}\right)=f X|D|^{-n}-\frac{1}{2} f\left(\nabla(X)+X^{2}\right)|D|^{-2-n} .
$$

Moreover, using $f \nabla(X)|D|^{-k}=0$ for any $k \geq 0$ since $f$ is a trace,

$$
\operatorname{Res}_{s=n-2} \operatorname{Tr}\left(\varepsilon(Y)|D|^{-s}\right)=\operatorname{Res}_{s=n-2} \operatorname{Tr}\left(\nabla(X) D^{-4}|D|^{-s}\right)=f \nabla(X)|D|^{-2-n}=0 .
$$

Similarly, since $Y \sim X D^{-2} \bmod O P^{-2}$ and $Y^{2} \sim X^{2} D^{-4} \bmod O P^{-3}$, we get

$$
\underset{s=n-2}{\operatorname{Res}} \operatorname{Tr}\left(Y^{2}|D|^{-s}\right)=\operatorname{Res}_{s=n-2} \operatorname{Tr}\left(X^{2} D^{-4}|D|^{-s}\right)=f X^{2}|D|^{-2-n} .
$$

Thus,

$$
\begin{aligned}
\operatorname{Res}_{s=n-2} \zeta_{D_{A}}(s)= & \operatorname{Res}_{s=n-2} \zeta_{D}(s) \\
& +\left(-\frac{n-2}{2}\right)\left(f X|D|^{-n}-\frac{1}{2} f\left(\nabla(X)+X^{2}\right)|D|^{-2-n}\right) \\
& +\frac{1}{2}\left(\frac{n-2}{2}\right)^{2} f \nabla(X)|D|^{-2-n}+\frac{1}{2}\left(\frac{n-2}{2}\right)^{2} f X^{2}|D|^{-2-n}
\end{aligned}
$$

Finally,

$$
\begin{aligned}
\operatorname{Res}_{s=n-2} \zeta_{D_{A}}(s)= & \operatorname{Res}_{s=n-2} \zeta_{D}(s)+\left(-\frac{n-2}{2}\right)\left(f X|D|^{-n}-\frac{1}{2} f X^{2}|D|^{-2-n}\right) \\
& +\frac{1}{2}\left(\frac{n-2}{2}\right)^{2} f X^{2}|D|^{-2-n},
\end{aligned}
$$

and the result follows from Proposition 4.8. 
Corollary 4.11. If the spectral triple is simple and satisfies the identity $f|D|^{-(n-2)}=$ $f \tilde{A} \mathcal{D}|D|^{-n}=f \mathcal{D} \tilde{A}|D|^{-n}=0$, then

$$
f\left|D_{A}\right|^{-(n-2)}=\frac{n(n-2)}{4}\left(f \tilde{A} \mathcal{D} \tilde{A} \mathcal{D}|D|^{-n-2}+\frac{n-2}{n} f \tilde{A}^{2}|D|^{-n}\right) .
$$

Proof. By the previous lemma, we have

$$
\begin{aligned}
\operatorname{Res}_{s=n-2} \zeta_{D_{A}}(s)= & \frac{n-2}{2}\left(-f \tilde{A}^{2}|D|^{-n}\right. \\
& \left.+\frac{n}{4} f\left(\tilde{A} \mathcal{D} \tilde{A} \mathcal{D}+\mathcal{D} \tilde{A} \mathcal{D} \tilde{A}+\tilde{A} \mathcal{D}^{2} \tilde{A}+\mathcal{D} \tilde{A}^{2} \mathcal{D}\right)|D|^{-n-2}\right)
\end{aligned}
$$

Since $\nabla(\tilde{A}) \in O P^{1}$, the trace property of $f$ yields the result.

\section{The noncommutative torus}

5.1. Notations. Let $C^{\infty}\left(\mathbb{T}_{\Theta}^{n}\right)$ be the smooth noncommutative $n$-torus associated to a non-zero skew-symmetric deformation matrix $\Theta \in M_{n}(\mathbb{R})$ (see [5], [30]). This means that $C^{\infty}\left(\mathbb{\mathbb { T }}_{\Theta}^{n}\right)$ is the algebra generated by $n$ unitaries $u_{i}, i=1, \ldots, n$, subject to the relations

$$
u_{i} u_{j}=e^{i \Theta_{i j}} u_{j} u_{i}
$$

and with Schwartz coefficients: an element $a \in C^{\infty}\left(\mathbb{T}_{\Theta}^{n}\right)$ can be written as $a=$ $\sum_{k \in \mathbb{Z}^{n}} a_{k} U_{k}$, where $\left\{a_{k}\right\} \in \mathcal{S}\left(\mathbb{Z}^{n}\right)$ with the Weyl elements defined by $U_{k}:=$ $e^{-\frac{i}{2} k \cdot \chi k} u_{1}^{k_{1}} \ldots u_{n}^{k_{n}}, k \in \mathbb{Z}^{n}$. Relation (5.1) reads

$$
U_{k} U_{q}=e^{-\frac{i}{2} k \cdot \Theta q} U_{k+q} \text { and } U_{k} U_{q}=e^{-i k \cdot \Theta q} U_{q} U_{k}
$$

where $\chi$ is the matrix restriction of $\Theta$ to its upper triangular part. Thus unitary operators $U_{k}$ satisfy $U_{k}^{*}=U_{-k}$ and $\left[U_{k}, U_{l}\right]=-2 i \sin \left(\frac{1}{2} k \cdot \Theta l\right) U_{k+l}$.

Let $\tau$ be the trace on $C^{\infty}\left(\mathbb{T}_{\Theta}^{n}\right)$ defined by $\tau\left(\sum_{k \in \mathbb{Z}^{n}} a_{k} U_{k}\right):=a_{0}$ and $\mathcal{H}_{\tau}$ be the GNS Hilbert space obtained by completion of $C^{\infty}\left(\mathbb{T}_{\Theta}^{n}\right)$ with respect to the norm induced by the scalar product $\langle a, b\rangle:=\tau\left(a^{*} b\right)$. On $\mathcal{H}_{\tau}=\left\{\sum_{k \in \mathbb{Z}^{n}} a_{k} U_{k} \mid\left\{a_{k}\right\}_{k} \in\right.$ $\left.l^{2}\left(\mathbb{Z}^{n}\right)\right\}$ we consider the left and right regular representations of $C^{\infty}\left(\mathbb{T}_{\Theta}^{n}\right)$ by bounded operators, which we denote by $L(\cdot)$ and $R(\cdot)$, respectively.

Let also $\delta_{\mu}, \mu \in\{1, \ldots, n\}$, be the $n$ (pairwise commuting) canonical derivations defined by

$$
\delta_{\mu}\left(U_{k}\right):=i k_{\mu} U_{k}
$$

We need to fix notations: let $\mathcal{A}_{\Theta}:=C^{\infty}\left(\mathbb{T}_{\Theta}^{n}\right)$ acting on $\mathcal{H}:=\mathcal{H}_{\tau} \otimes \mathbb{C}^{2^{m}}$ with $n=2 m$ or $n=2 m+1$ (i.e., $m=\left\lfloor\frac{n}{2}\right\rfloor$ is the integer part of $\frac{n}{2}$ ), the square integrable sections of the trivial spin bundle over $\mathbb{T}^{n}$. 
Each element of $\mathcal{A}_{\Theta}$ is represented on $\mathcal{H}$ as $L(a) \otimes 1_{2^{m}}$ where $L$ (resp. $R$ ) is the left (resp. right) multiplication. The Tomita conjugation $J_{0}(a):=a^{*}$ satisfies $\left[J_{0}, \delta_{\mu}\right]=0$ and we define $J:=J_{0} \otimes C_{0}$ where $C_{0}$ is an operator on $\mathbb{C}^{2^{m}}$. The Dirac operator is given by

$$
\mathcal{D}:=-i \delta_{\mu} \otimes \gamma^{\mu}
$$

where we use hermitian Dirac matrices $\gamma$. It is defined and symmetric on the dense subset of $\mathcal{H}$ given by $C^{\infty}\left(\mathbb{T}_{\Theta}^{n}\right) \otimes \mathbb{C}^{2^{m}}$. We still denote $\mathcal{D}$ its selfadjoint extension. This implies that

$$
C_{0} \gamma^{\alpha}=-\varepsilon \gamma^{\alpha} C_{0}
$$

and

$$
\mathcal{D} U_{k} \otimes e_{i}=k_{\mu} U_{k} \otimes \gamma^{\mu} e_{i},
$$

where $\left(e_{i}\right)$ is the canonical basis of $\mathbb{C}^{2^{m}}$. Moreover, $C_{0}^{2}= \pm 1_{2^{m}}$, depending on the parity of $m$. Finally, one introduces the chirality (which in the even case is $\left.\chi:=\mathrm{id} \otimes(-i)^{m} \gamma^{1} \ldots \gamma^{n}\right)$ and this yields that $\left(\mathcal{A}_{\Theta}, \mathcal{H}, \mathcal{D}, J, \chi\right)$ satisfies all axioms of a spectral triple; see [7], [23].

The Dirac operator $V_{u} \mathcal{D} V_{u}^{*}$ perturbed by the unitary

$$
V_{u}:=\left(L(u) \otimes 1_{2^{m}}\right) J\left(L(u) \otimes 1_{2^{m}}\right) J^{-1},
$$

defined by $u u^{*}=u^{*} u=U_{0}$ for every unitary $u \in \mathcal{A}$, must satisfy condition (1.3) (which is equivalent to $\mathcal{H}$ being endowed with a structure of $\mathcal{A}_{\Theta}$-bimodule). This implies the existence of a symmetrized covariant Dirac operator,

$$
\mathcal{D}_{A}:=\mathcal{D}+A+\epsilon J A J^{-1},
$$

since $V_{u} \mathcal{D} V_{u}^{*}=\mathcal{D}_{L(u) \otimes 1_{2} m\left[\mathcal{D}, L\left(u^{*}\right) \otimes 1_{2^{m}}\right]}$ : in fact, for $a \in \mathcal{A}_{\Theta}$, using $J_{0} L(a) J_{0}^{-1}=$ $R\left(a^{*}\right)$, we obtain that

$$
\epsilon J\left(L(a) \otimes \gamma^{\alpha}\right) J^{-1}=-R\left(a^{*}\right) \otimes \gamma^{\alpha},
$$

and the representation $L$ and the anti-representation $R$ are $\mathbb{C}$-linear, commute and satisfy

$$
\left[\delta_{\alpha}, L(a)\right]=L\left(\delta_{\alpha} a\right), \quad\left[\delta_{\alpha}, R(a)\right]=R\left(\delta_{\alpha} a\right) .
$$

This induces some covariance property for the Dirac operator; one checks that

$$
L\left(U_{k}\right) \otimes 1_{2^{m}}\left[\mathcal{D}, L\left(U_{k}^{*}\right) \otimes 1_{2^{m}}\right]=1 \otimes\left(-k_{\mu} \gamma^{\mu}\right),
$$

for all $k \in \mathbb{Z}^{n}$. Thus, with (5.5), we get $U_{k}\left[\mathcal{D}, U_{k}^{*}\right]+\epsilon J U_{k}\left[\mathcal{D}, U_{k}^{*}\right] J^{-1}=0$ and

$$
V_{U_{k}} \mathcal{D} V_{U_{k}}^{*}=\mathcal{D}=\mathcal{D}_{L\left(U_{k}\right) \otimes 1_{2} m\left[\mathcal{D}, L\left(U_{k}^{*}\right) \otimes 1_{2} m\right]} .
$$


Moreover, we obtain the gauge transformation

$$
V_{u} \mathcal{D}_{A} V_{u}^{*}=\mathcal{D}_{\gamma_{u}(A)},
$$

where the gauged transform 1-form of $A$ is

$$
\gamma_{u}(A):=u\left[\mathcal{D}, u^{*}\right]+u A u^{*}
$$

with the shorthand $L(u) \otimes 1_{2^{m}} \rightarrow u$.

As a consequence, the spectral action is gauge invariant:

$$
\mathcal{S}\left(\mathcal{D}_{A}, \Phi, \Lambda\right)=\mathcal{S}\left(\mathcal{D}_{\gamma_{u}(A)}, \Phi, \Lambda\right)
$$

An arbitrary selfadjoint 1 -form $A$ can be written as

$$
A=L\left(-i A_{\alpha}\right) \otimes \gamma^{\alpha} \text { with } A_{\alpha}=-A_{\alpha}^{*} \in \mathcal{A}_{\Theta},
$$

thus

$$
\mathcal{D}_{A}=-i\left(\delta_{\alpha}+L\left(A_{\alpha}\right)-R\left(A_{\alpha}\right)\right) \otimes \gamma^{\alpha}
$$

Defining

$$
\tilde{A}_{\alpha}:=L\left(A_{\alpha}\right)-R\left(A_{\alpha}\right)
$$

we get $\mathcal{D}_{A}^{2}=-g^{\alpha_{1} \alpha_{2}}\left(\delta_{\alpha_{1}}+\tilde{A}_{\alpha_{1}}\right)\left(\delta_{\alpha_{2}}+\tilde{A}_{\alpha_{2}}\right) \otimes 1_{2^{m}}-\frac{1}{2} \Omega_{\alpha_{1} \alpha_{2}} \otimes \gamma^{\alpha_{1} \alpha_{2}}$, where

$$
\begin{aligned}
\gamma^{\alpha_{1} \alpha_{2}} & :=\frac{1}{2}\left(\gamma^{\alpha_{1}} \gamma^{\alpha_{2}}-\gamma^{\alpha_{2}} \gamma^{\alpha_{1}}\right), \\
\Omega_{\alpha_{1} \alpha_{2}} & :=\left[\delta_{\alpha_{1}}+\tilde{A}_{\alpha_{1}}, \delta_{\alpha_{2}}+\tilde{A}_{\alpha_{2}}\right]=L\left(F_{\alpha_{1} \alpha_{2}}\right)-R\left(F_{\alpha_{1} \alpha_{2}}\right)
\end{aligned}
$$

with

$$
F_{\alpha_{1} \alpha_{2}}:=\delta_{\alpha_{1}}\left(A_{\alpha_{2}}\right)-\delta_{\alpha_{2}}\left(A_{\alpha_{1}}\right)+\left[A_{\alpha_{1}}, A_{\alpha_{2}}\right]
$$

In summary,

$$
\begin{gathered}
\mathcal{D}_{A}^{2}=-\delta^{\alpha_{1} \alpha_{2}}\left(\delta_{\alpha_{1}}+L\left(A_{\alpha_{1}}\right)-R\left(A_{\alpha_{1}}\right)\right)\left(\delta_{\alpha_{2}}+L\left(A_{\alpha_{2}}\right)-R\left(A_{\alpha_{2}}\right)\right) \\
\otimes 1_{2^{m}}-\frac{1}{2}\left(L\left(F_{\alpha_{1} \alpha_{2}}\right)-R\left(F_{\alpha_{1} \alpha_{2}}\right)\right) \otimes \gamma^{\alpha_{1} \alpha_{2}}
\end{gathered}
$$

5.2. Kernels and dimension spectrum. We now compute the kernel of the perturbed Dirac operator.

Proposition 5.1. (i) $\operatorname{Ker} \mathcal{D}=U_{0} \otimes \mathbb{C}^{2^{m}}$, so $\operatorname{dim} \operatorname{Ker} \mathcal{D}=2^{m}$.

(ii) For any selfadjoint 1-form $A$ we have $\operatorname{Ker} \mathcal{D} \subseteq \operatorname{Ker} \mathcal{D}_{A}$.

(ii) For any unitary $u \in \mathcal{A}$ we have $\operatorname{Ker} \mathcal{D}_{\gamma_{u}(A)}=V_{u} \operatorname{Ker} \mathcal{D}_{A}$. 
Proof. (i) Let $\psi=\sum_{k, j} c_{k, j} U_{k} \otimes e_{j} \in \operatorname{Ker} \mathcal{D}$. Then $0=\mathcal{D}^{2} \psi=\sum_{k, i} c_{k, j}|k|^{2} U_{k} \otimes$ $e_{j}$, which implies that $c_{k, j}|k|^{2}=0$ for any $k \in \mathbb{Z}^{n}$ and $1 \leq j \leq 2^{m}$. The result follows.

(ii) Let $\psi \in \operatorname{Ker} \mathcal{D}$. Then $\psi=U_{0} \otimes v$ with $v \in \mathbb{C}^{2^{m}}$, and from (5.11) we get

$$
\mathcal{D}_{A} \psi=\mathcal{D} \psi+\left(A+\epsilon J A J^{-1}\right) \psi=\left(A+\epsilon J A J^{-1}\right) \psi=-i\left[A_{\alpha}, U_{0}\right] \otimes \gamma^{\alpha} v=0
$$

since $U_{0}$ is the unit of the algebra, which proves that $\psi \in \operatorname{Ker} \mathcal{D}_{A}$.

(iii) This is a direct consequence of (5.8).

Corollary 5.2. Let $A$ be a selfadjoint 1-form. Then $\operatorname{Ker} \mathcal{D}_{A}=\operatorname{Ker} \mathcal{D}$ in the following cases:

(i) $A_{u}:=L(u) \otimes 1_{2^{m}}\left[\mathcal{D}, L\left(u^{*}\right) \otimes 1_{2^{m}}\right]$ when $u$ is a unitary in $\mathcal{A}$.

(ii) $\|A\|<\frac{1}{2}$.

(iii) The matrix $\frac{1}{2 \pi} \Theta$ has only integral coefficients.

Proof. (i) This follows from previous result because $V_{u}\left(U_{0} \otimes v\right)=U_{0} \otimes v$ for any $v \in \mathbb{C}^{2^{m}}$.

(ii) Let $\psi=\sum_{k, j} c_{k, j} U_{k} \otimes e_{j}$ be in $\operatorname{Ker} \mathcal{D}_{A}\left(\right.$ so $\left.\sum_{k, j}\left|c_{k, j}\right|^{2}<\infty\right)$ and $\phi:=$ $\sum_{j} c_{0, j} U_{0} \otimes e_{j}$. Thus $\psi^{\prime}:=\psi-\phi \in \operatorname{Ker} \mathcal{D}_{A}$ since $\phi \in \operatorname{Ker} \mathcal{D} \subseteq \operatorname{Ker} \mathcal{D}_{A}$ and

$$
\begin{aligned}
\left\|\sum_{0 \neq k \in \mathbb{Z}^{n}, j} c_{k, j} k_{\alpha} U_{k} \otimes \gamma^{\alpha} e_{j}\right\|^{2} & =\left\|\mathcal{D} \psi^{\prime}\right\|^{2} \\
& =\left\|-\left(A+\epsilon J A J^{-1}\right) \psi^{\prime}\right\|^{2} \leq 4\|A\|^{2}\left\|\psi^{\prime}\right\|^{2}<\left\|\psi^{\prime}\right\|^{2} .
\end{aligned}
$$

Let $X_{k}:=\sum_{\alpha} k_{\alpha} \gamma_{\alpha}$. Then $X_{k}^{2}=\sum_{\alpha}\left|k_{\alpha}\right|^{2} 1_{2^{m}}$ is invertible and the vectors $\left\{U_{k} \otimes X_{k} e_{j}\right\}_{0 \neq k \in \mathbb{Z}^{n}, j}$ are orthogonal in $\mathcal{H}$, so

$$
\sum_{0 \neq k \in \mathbb{Z}^{n}, j}\left(\sum_{\alpha}\left|k_{\alpha}\right|^{2}\right)\left|c_{k, j}\right|^{2}<\sum_{0 \neq k \in \mathbb{Z}^{n}, j}\left|c_{k, j}\right|^{2},
$$

which is possible only if $c_{k, j}=0$ for all $k, j$, that is, $\psi^{\prime}=0$ and $\psi=\phi \in \operatorname{Ker} \mathcal{D}$.

(iii) This is a consequence of the fact that the algebra is commutative, and hence $A+\epsilon J A J^{-1}=0$.

Note that if $\tilde{A}_{u}:=A_{u}+\epsilon J A_{u} J^{-1}$, then, by (5.6), $\tilde{A}_{U_{k}}=0$ for all $k \in \mathbb{Z}^{n}$ and $\left\|A_{U_{k}}\right\|=|k|$, but for an arbitrary unitary $u \in \mathcal{A}, \tilde{A}_{u} \neq 0$ and hence $\mathcal{D}_{A_{u}} \neq \mathcal{D}$.

Naturally the above result is also a direct consequence of the fact that the eigenspace of an isolated eigenvalue of an operator is not modified by small perturbations. However, it is interesting to compute the last result directly to emphasize the difficulty of the general case. 
So let $\psi=\sum_{l \in \mathbb{Z}^{n}, 1 \leq j \leq 2^{m}} c_{l, j} U_{l} \otimes e_{j} \in \operatorname{Ker} \mathcal{D}_{A}$. Then $\sum_{l \in \mathbb{Z}^{n}, 1 \leq j \leq 2^{m}}\left|c_{l, j}\right|^{2}<$ $\infty$. We have to show that $\psi \in \operatorname{Ker} \mathcal{D}$ that is $c_{l, j}=0$ when $l \neq 0$.

Taking the scalar product of $\left\langle U_{k} \otimes e_{i}\right|$ with

$$
0=\mathcal{D}_{A} \psi=\sum_{l, \alpha, j} c_{l, j}\left(l^{\alpha} U_{l}-i\left[A_{\alpha}, U_{l}\right]\right) \otimes \gamma^{\alpha} e_{j},
$$

we obtain that

$$
0=\sum_{l, \alpha, j} c_{l, j}\left(l^{\alpha} \delta_{k, l}-i\left\langle U_{k},\left[A_{\alpha}, U_{l}\right]\right\rangle\right)\left\langle e_{i}, \gamma^{\alpha} e_{j}\right\rangle
$$

If $A_{\alpha}=\sum_{\alpha, l} a_{\alpha, l} U_{l} \otimes \gamma^{\alpha},\left\{a_{\alpha, l}\right\}_{l} \in \mathcal{S}\left(\mathbb{Z}^{n}\right)$, then it follows that $\left[U_{l}, U_{m}\right]=$ $-2 i \sin \left(\frac{1}{2} l \cdot \Theta m\right) U_{l+m}$ and

$$
\begin{aligned}
\left\langle U_{k},\left[A_{\alpha}, U_{l}\right]\right\rangle & =\sum_{l^{\prime} \in \mathbb{Z}^{n}} a_{\alpha, l^{\prime}}\left(-2 i \sin \left(\frac{1}{2} l^{\prime} \cdot \Theta l\right)\right)\left\langle U_{k}, U_{l^{\prime}+l}\right\rangle \\
& =-2 i a_{\alpha, k-l} \sin \left(\frac{1}{2} k \cdot \Theta l\right) .
\end{aligned}
$$

Thus

$$
0=\sum_{l \in \mathbb{Z}^{n}} \sum_{\alpha=1}^{n} \sum_{j=1}^{2^{m}} c_{l, j}\left(l^{\alpha} \delta_{k, l}-2 a_{\alpha, k-l} \sin \left(\frac{1}{2} k \cdot \Theta l\right)\right)\left\langle e_{i}, \gamma^{\alpha} e_{j}\right\rangle
$$

for all $k \in \mathbb{Z}^{n}$ and for all $i$ with $1 \leq i \leq 2^{m}$.

We conjecture that $\operatorname{Ker} \mathcal{D}=\operatorname{Ker} \mathcal{D}_{A}$ at least for generic $\Theta$ 's: the constraints (5.14) should imply $c_{l, j}=0$ for all $j$ and all $l \neq 0$, which means that $\psi \in \operatorname{Ker} \mathcal{D}$. If $\frac{1}{2 \pi} \Theta$ has only integer coefficients, then the sin part of these constraints disappears, which gives the result.

Lemma 5.3. If $\frac{1}{2 \pi} \Theta$ is diophantine, then $\operatorname{Sp}\left(C^{\infty}\left(\mathbb{\nabla}_{\Theta}^{n}\right), \mathcal{H}, \mathcal{D}\right)=\mathbb{Z}$, and all these poles are simple.

Proof. Let $B \in \mathcal{D}(\mathcal{A})$ and $p \in \mathbb{N}_{0}$. Suppose that $B$ is of the form

$$
B=a_{r} b_{r} \mathcal{D}^{q_{r-1}}|\mathcal{D}|^{p_{r-1}} a_{r-1} b_{r-1} \ldots \mathcal{D}^{q_{1}}|\mathcal{D}|^{p_{1}} a_{1} b_{1}
$$

where $r \in \mathbb{N}, a_{i} \in \mathcal{A}, b_{i} \in J \mathcal{A} J^{-1}, q_{i}, p_{i} \in \mathbb{N}_{0}$. We denote $a_{i}=: \sum_{l} a_{i, l} U_{l}$ and $b_{i}=: \sum_{i} b_{i, l} U_{l}$. With the shorthand $k_{\mu_{1}, \mu_{q_{i}}}:=k_{\mu_{1}} \ldots k_{\mu_{q_{i}}}$ and $\gamma^{\mu_{1}, \mu_{q_{i}}}=$ $\gamma^{\mu_{1}} \ldots \gamma^{\mu_{q_{i}} \text {, we get }}$

$$
\begin{aligned}
\mathcal{D}^{q_{1}}|\mathcal{D}|^{p_{1}} a_{1} b_{1} U_{k} \otimes e_{j} \\
\quad=\sum_{l_{1}, l_{1}^{\prime}} a_{1, l_{1}} b_{1, l_{1}^{\prime}} U_{l_{1}} U_{k} U_{l_{1}^{\prime}}\left|k+l_{1}+l_{1}^{\prime}\right|^{p_{1}}\left(k+l_{1}+l_{1}^{\prime}\right)_{\mu_{1}, \mu_{q_{1}}} \otimes \gamma^{\mu_{1}, \mu_{q_{1}}} e_{j},
\end{aligned}
$$


which gives after $r$ iterations

$$
\begin{aligned}
B U_{k} \otimes e_{j}= & \sum_{l, l^{\prime}} \tilde{a}_{l} \tilde{b}_{l} U_{l_{r}} \ldots U_{l_{1}} U_{k} U_{l_{1}^{\prime}} \ldots U_{l_{r}^{\prime}} \\
& \prod_{i=1}^{r-1}\left|k+\hat{l}_{i}+\hat{l}_{i}^{\prime}\right|^{p_{i}}\left(k+\hat{l}_{i}+\hat{l}_{i}^{\prime}\right)_{\mu_{1}^{i}, \mu_{q_{i}}^{i}} \otimes \gamma^{\mu_{1}^{r-1}, \mu_{q_{r-1}-1}^{r-1} \ldots \gamma^{\mu_{1}^{1}, \mu_{q_{1}}^{1}} e_{j},}
\end{aligned}
$$

where $\tilde{a}_{l}:=a_{1, l_{1}} \ldots a_{r, l_{r}}$ and $\tilde{b}_{l^{\prime}}:=b_{1, l_{1}^{\prime}} \ldots b_{r, l_{r}^{\prime}}$.

Let us write $F_{\mu}\left(k, l, l^{\prime}\right):=\prod_{i=1}^{r-1}\left|k+\hat{l}_{i}+\hat{l}_{i}^{\prime}\right|^{p_{i}}\left(k+\hat{l}_{i}+\hat{l}_{i}^{\prime}\right)_{\mu_{1}^{i}, \mu_{q_{i}}^{i}}$ and $\gamma^{\mu}:=$

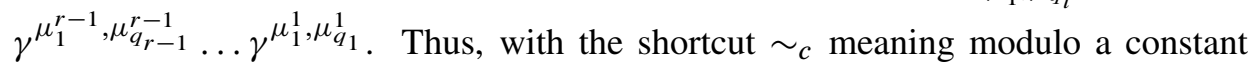
function in the variable $s$, we have

$$
\begin{aligned}
& \operatorname{Tr}\left(B|D|^{-2 p-s}\right) \\
& \quad \sim_{c} \sum_{k}^{\prime} \sum_{l, l^{\prime}} \tilde{a}_{l} \tilde{b}_{l^{\prime}} \tau\left(U_{-k} U_{l_{r}} \ldots U_{l_{1}} U_{k} U_{l_{1}^{\prime}} \ldots U_{l_{r}^{\prime}}\right) \frac{F_{\mu}\left(k, l, l^{\prime}\right)}{|k|^{s+2 p}} \operatorname{Tr}\left(\gamma^{\mu}\right) .
\end{aligned}
$$

Since $U_{l_{r}} \ldots U_{l_{1}} U_{k}=U_{k} U_{l_{r}} \ldots U_{l_{1}} e^{-i \sum_{1}^{r} l_{i} . \Theta k}$ we get

$$
\tau\left(U_{-k} U_{l_{r}} \ldots U_{l_{1}} U_{k} U_{l_{1}^{\prime}} \ldots U_{l_{r}^{\prime}}\right)=\delta_{\sum_{1}^{r} l_{i}+l_{i}^{\prime}, 0} e^{i \phi\left(l, l^{\prime}\right)} e^{-i \sum_{1}^{r} l_{i} \cdot \Theta k},
$$

where $\phi$ is a real valued function. Thus,

$$
\begin{aligned}
\operatorname{Tr}\left(B|D|^{-2 p-s}\right) & \sim_{c} \sum_{k}^{\prime} \sum_{l, l^{\prime}} e^{i \phi\left(l, l^{\prime}\right)} \delta_{\sum_{1}^{r} l_{i}+l_{i}^{\prime}, 0} \tilde{a}_{l} \tilde{b}_{l^{\prime}} \frac{F_{\mu}\left(k, l, l^{\prime}\right) e^{-i \sum_{1}^{r} l_{i} \cdot \Theta k}}{|k|^{s+2 p}} \operatorname{Tr}\left(\gamma^{\mu}\right) \\
& \sim_{c} f_{\mu}(s) \operatorname{Tr}\left(\gamma^{\mu}\right) .
\end{aligned}
$$

The function $f_{\mu}(s)$ can be decomposed into a linear combination of zeta functions of the type described in Theorem 2.18 (resp. Theorem 2.6 if $r=1$ or all the $p_{i}$ are zero). Thus, $s \mapsto \operatorname{Tr}\left(B|D|^{-2 p-s}\right)$ has only poles in $\mathbb{Z}$ and each pole is simple. Finally, by linearity, the result follows.

The dimension spectrum of the noncommutative torus is simple:

Proposition 5.4. (i) If $\frac{1}{2 \pi} \Theta$ is diophantine, then the dimension spectrum of the triple $\left(C^{\infty}\left(\mathbb{\nabla}_{\Theta}^{n}\right), \mathcal{H}, \mathcal{D}\right)$ is equal to the set $\left\{n-k \mid k \in \mathbb{N}_{0}\right\}$, and all these poles are simple.

(ii) $\zeta_{D}(0)=0$.

Proof. (i) This follows from Lemma 5.3 and Remark 3.9.

(ii) We have $\zeta_{D}(s)=\sum_{k \in \mathbb{Z}^{n}} \sum_{1 \leq j \leq 2^{m}}\left\langle U_{k} \otimes e_{j},|D|^{-s} U_{k} \otimes e_{j}\right\rangle=$ $2^{m}\left(\sum_{k \in \mathbb{Z}^{n}}^{\prime} \frac{1}{|k|^{s}}+1\right)=2^{m}\left(Z_{n}(s)+1\right)$. The result follows from (2.15).

We have computed $\zeta_{D}(0)$ relatively easy, but the main difficulty of the present work is essentially to calculate $\zeta_{D_{A}}(0)$. 
5.3. Noncommutative integral computations. We fix a selfadjoint 1 -form $A$ on the noncommutative torus of dimension $n$.

Proposition 5.5. If $\frac{1}{2 \pi} \Theta$ is diophantine, then the first elements of the expansion (1.5) are given by

$$
\begin{aligned}
& f\left|D_{A}\right|^{-n}=f|D|^{-n}=2^{m+1} \pi^{n / 2} \Gamma\left(\frac{n}{2}\right)^{-1}, \\
& f\left|D_{A}\right|^{n-k}=0 \text { for } k \text { odd }, \\
& f\left|D_{A}\right|^{n-2}=0 .
\end{aligned}
$$

We need few technical lemmas.

Lemma 5.6. On the noncommutative torus, for any $t \in \mathbb{R}$,

$$
f \tilde{A} \mathcal{D}|D|^{-t}=f \mathcal{D} \tilde{A}|D|^{-t}=0 .
$$

Proof. Using the notation of (5.10), we have

$$
\begin{aligned}
\operatorname{Tr}\left(\tilde{A} \mathcal{D}|D|^{-s}\right) & \sim_{c} \sum_{j} \sum_{k}^{\prime}\left\langle U_{k} \otimes e_{j},-i k_{\mu}|k|^{-s}\left[A_{\alpha}, U_{k}\right] \otimes \gamma^{\alpha} \gamma^{\mu} e_{j}\right\rangle \\
& \sim_{c}-i \operatorname{Tr}\left(\gamma^{\alpha} \gamma^{\mu}\right) \sum_{k}^{\prime} k_{\mu}|k|^{-s}\left\langle U_{k},\left[A_{\alpha}, U_{k}\right]\right\rangle=0
\end{aligned}
$$

since $\left\langle U_{k},\left[A_{\alpha}, U_{k}\right]\right\rangle=0$. Similarly

$$
\begin{aligned}
& \operatorname{Tr}\left(\mathcal{D} \tilde{A}|D|^{-s}\right) \\
& \quad \sim_{c} \sum_{j} \sum_{k}^{\prime}\left\langle U_{k} \otimes e_{j},|k|^{-s} \sum_{l} a_{\alpha, l} 2 \sin \frac{k \cdot \Theta l}{2}(l+k)_{\mu} U_{l+k} \otimes \gamma^{\mu} \gamma^{\alpha} e_{j}\right\rangle \\
& \quad \sim_{c} 2 \operatorname{Tr}\left(\gamma^{\mu} \gamma^{\alpha}\right) \sum_{k}^{\prime} \sum_{l} a_{\alpha, l} \sin \frac{k \cdot \Theta l}{2}(l+k)_{\mu}|k|^{-s}\left\langle U_{k}, U_{l+k}\right\rangle=0 .
\end{aligned}
$$

Any element $h$ in the algebra generated by $\mathcal{A}$ and $[\mathcal{D}, \mathcal{A}]$ can be written as a linear combination of terms of the form $a_{1} p_{1} \ldots a_{n}{ }^{p_{r}}$, where $a_{i}$ are elements of $\mathcal{A}$ or $[\mathcal{D}, \mathcal{A}]$. Such a term can be written as a series $b:=\sum a_{1, \alpha_{1}, l_{1}} \ldots a_{q, \alpha_{q}, l_{q}} U_{l_{1}} \ldots U_{l_{q}} \otimes$ $\gamma^{\alpha_{1}} \ldots \gamma^{\alpha_{q}}$, where $a_{i, \alpha_{i}}$ are Schwartz sequences and when $a_{i}=: \sum_{l} a_{l} U_{l} \in \mathcal{A}$ we set $a_{i, \alpha, l}=a_{i, l}$ with $\gamma^{\alpha}=1$. We define

$$
L(b):=\tau\left(\sum_{l} a_{1, \alpha_{1}, l_{1}} \ldots a_{q, \alpha_{q}, l_{q}} U_{l_{1}} \ldots U_{l_{q}}\right) \operatorname{Tr}\left(\gamma^{\alpha_{1}} \ldots \gamma^{\alpha_{q}}\right) .
$$

By linearity, $L$ is defined as a linear form on the whole algebra generated by $\mathcal{A}$ and $[\mathcal{D}, \mathcal{A}]$. 
Lemma 5.7. If $h$ is an element of the algebra generated by $\mathcal{A}$ and $[\mathcal{D}, \mathcal{A}]$, then

$$
\operatorname{Tr}\left(h|D|^{-s}\right) \sim_{c} L(h) Z_{n}(s) .
$$

In particular, $\operatorname{Tr}\left(h|D|^{-s}\right)$ has at most one pole at $s=n$.

Proof. With $b$ of the form $\sum a_{1, \alpha_{1}, l_{1}} \ldots a_{q, \alpha_{q}, l_{q}} U_{l_{1}} \ldots U_{l_{q}} \otimes \gamma^{\alpha_{1}} \ldots \gamma^{\alpha_{q}}$, we get

$$
\begin{aligned}
& \operatorname{Tr}\left(b|D|^{-s}\right) \\
& \quad \sim_{c} \sum_{k \in \mathbb{Z}^{n}}^{\prime}\left\langle U_{k}, \sum_{l} a_{1, \alpha_{1}, l_{1}} \ldots a_{q, \alpha_{q}, l_{q}} U_{l_{1}} \ldots U_{l_{q}} U_{k}\right\rangle \operatorname{Tr}\left(\gamma^{\alpha_{1}} \ldots \gamma^{\alpha_{q}}\right)|k|^{-s} \\
& \quad \sim_{c} \tau\left(\sum_{l} a_{1, \alpha_{1}, l_{1}} \ldots a_{q, \alpha_{q}, l_{q}} U_{l_{1}} \ldots U_{l_{q}}\right) \operatorname{Tr}\left(\gamma^{\alpha_{1}} \ldots \gamma^{\alpha_{q}}\right) Z_{n}(s)=L(b) Z_{n}(s) .
\end{aligned}
$$

The result now follows from linearity of the trace.

Lemma 5.8. If $\frac{1}{2 \pi} \Theta$ is diophantine, the function $s \mapsto \operatorname{Tr}\left(\varepsilon J A J^{-1} A|D|^{-s}\right)$ extends meromorphically on the whole plane with only one possible pole at $s=n$. Moreover, this pole is simple and

$$
\operatorname{Res}_{s=n} \operatorname{Tr}\left(\varepsilon J A J^{-1} A|D|^{-s}\right)=a_{\alpha, 0} a_{0}^{\alpha} 2^{m+1} \pi^{n / 2} \Gamma(n / 2)^{-1} .
$$

Proof. With $A=L\left(-i A_{\alpha}\right) \otimes \gamma^{\alpha}$, we get $\epsilon J A J^{-1}=R\left(i A_{\alpha}\right) \otimes \gamma^{\alpha}$, and by multiplication $\varepsilon J A J^{-1} A=R\left(A_{\beta}\right) L\left(A_{\alpha}\right) \otimes \gamma^{\beta} \gamma^{\alpha}$. Thus,

$$
\begin{aligned}
\operatorname{Tr}\left(\varepsilon J A J^{-1} A|D|^{-s}\right) & \sim_{c} \sum_{k \in \mathbb{Z}^{n}}^{\prime}\left\langle U_{k}, A_{\alpha} U_{k} A_{\beta}\right\rangle|k|^{-s} \operatorname{Tr}\left(\gamma^{\beta} \gamma^{\alpha}\right) \\
& \sim_{c} \sum_{k \in \mathbb{Z}^{n}}^{\prime} \sum_{l} a_{\alpha, l} a_{\beta,-l} e^{i k \cdot \Theta l}|k|^{-s} \operatorname{Tr}\left(\gamma^{\beta} \gamma^{\alpha}\right) \\
& \sim_{c} 2^{m} \sum_{k \in \mathbb{Z}^{n}}^{\prime} \sum_{l} a_{\alpha, l} a_{-l}^{\alpha} e^{i k \cdot \Theta l}|k|^{-s} .
\end{aligned}
$$

Theorem 2.6 (ii) entails that $\sum_{k \in \mathbb{Z}^{n}}^{\prime} \sum_{l} a_{\alpha, l} a_{-l}^{\alpha} e^{i k \cdot \Theta l}|k|^{-s}$ extends meromorphically to the whole plane $\mathbb{C}$ with only one possible pole at $s=n$. Moreover, this pole is simple and we have

$$
\operatorname{Res}_{s=n} \sum_{k \in \mathbb{Z}^{n}}^{\prime} \sum_{l} a_{\alpha, l} a_{-l}^{\alpha} e^{i k \cdot \Theta l}|k|^{-s}=a_{\alpha, 0} a_{0}^{\alpha} \operatorname{Res}_{s=n} Z_{n}(s) .
$$

Equation (2.14) now gives the result.

Lemma 5.9. If $\frac{1}{2 \pi} \Theta$ is diophantine, then for any $t \in \mathbb{R}$,

$$
f X|D|^{-t}=\delta_{t, n} 2^{m+1}\left(-\sum_{l} a_{\alpha, l} a_{-l}^{\alpha}+a_{\alpha, 0} a_{0}^{\alpha}\right) 2 \pi^{n / 2} \Gamma(n / 2)^{-1} \text {. }
$$

where $X=\tilde{A} \mathcal{D}+\mathcal{D} \tilde{A}+\tilde{A}^{2}$ and $A=:-i \sum_{l} a_{\alpha, l} U_{l} \otimes \gamma^{\alpha}$. 
Proof. By Lemma 5.6, we get $f X|D|^{-t}=\operatorname{Res}_{s=0} \operatorname{Tr}\left(\tilde{A}^{2}|D|^{-s-t}\right)$. Since $A$ and $\varepsilon J A J^{-1}$ commute, we have $\tilde{A}^{2}=A^{2}+J A^{2} J^{-1}+2 \varepsilon J A J^{-1} A$. Thus,

$\operatorname{Tr}\left(\tilde{A}^{2}|D|^{-s-t}\right)=\operatorname{Tr}\left(A^{2}|D|^{-s-t}\right)+\operatorname{Tr}\left(J A^{2} J^{-1}|D|^{-s-t}\right)+2 \operatorname{Tr}\left(\varepsilon J A J^{-1} A|D|^{-s-t}\right)$.

Since $|D|$ and $J$ commute, we have with Lemma 5.7,

$$
\operatorname{Tr}\left(\tilde{A}^{2}|D|^{-s-t}\right) \sim_{c} 2 L\left(A^{2}\right) Z_{n}(s+t)+2 \operatorname{Tr}\left(\varepsilon J A J^{-1} A|D|^{-s-t}\right) .
$$

Thus Lemma 5.8 entails that $\operatorname{Tr}\left(\tilde{A}^{2}|D|^{-s-t}\right)$ is holomorphic at 0 if $t \neq n$. When $t=n$,

$$
\operatorname{Res}_{s=0} \operatorname{Tr}\left(\tilde{A}^{2}|D|^{-s-t}\right)=2^{m+1}\left(-\sum_{l} a_{\alpha, l} a_{-l}^{\alpha}+a_{\alpha, 0} a_{0}^{\alpha}\right) 2 \pi^{n / 2} \Gamma(n / 2)^{-1},
$$

which gives the result.

Lemma 5.10. If $\frac{1}{2 \pi} \Theta$ is diophantine, then

$$
f \tilde{A} \mathcal{D} \tilde{A} \mathcal{D}|D|^{-2-n}=-\frac{n-2}{n} f \tilde{A}^{2}|D|^{-n} .
$$

Proof. With $\mathcal{D} J=\varepsilon J \mathcal{D}$, we get

$$
f \tilde{A} \mathcal{D} \tilde{A} \mathcal{D}|D|^{-2-n}=2 f A \mathcal{D} A \mathcal{D}|D|^{-2-n}+2 f \varepsilon J A J^{-1} \mathcal{D} A \mathcal{D}|D|^{-2-n} .
$$

Let us first compute $f A \mathcal{D} A \mathcal{D}|D|^{-2-n}$. We have, with $A=:-i L\left(A_{\alpha}\right) \otimes \gamma^{\alpha}=$ : $-i \sum_{l} a_{\alpha, l} U_{l} \otimes \gamma^{\alpha}$

$$
\begin{aligned}
& \operatorname{Tr}\left(A \mathcal{D} A \mathcal{D}|D|^{-s-2-n}\right) \\
& \quad \sim_{c}-\sum_{k}^{\prime} \sum_{l_{1}, l_{2}} a_{\alpha_{2}, l_{2}} a_{\alpha_{1}, l_{1}} \tau\left(U_{-k} U_{l_{2}} U_{l_{1}} U_{k}\right) \frac{k_{\mu_{1}}\left(k+l_{1}\right) \mu_{2}}{|k|^{s+2+n}} \operatorname{Tr}\left(\gamma^{\alpha, \mu}\right)
\end{aligned}
$$

where $\gamma^{\alpha, \mu}:=\gamma^{\alpha_{2}} \gamma^{\mu_{2}} \gamma^{\alpha_{1}} \gamma^{\mu_{1}}$. Thus,

$$
f A \mathcal{D} A \mathcal{D}|D|^{-2-n}=-\sum_{l} a_{\alpha_{2},-l} a_{\alpha_{1}, l} \operatorname{Res}_{s=0}\left(\sum_{k}^{\prime} \frac{k_{\mu_{1}} k_{\mu_{2}}}{|k|^{s+2+n}}\right) \operatorname{Tr}\left(\gamma^{\alpha, \mu}\right) .
$$

We have also, with $\varepsilon J A J^{-1}=i R\left(A_{\alpha}\right) \otimes \gamma^{a}$,

$$
\begin{aligned}
& \operatorname{Tr}\left(\varepsilon J A J^{-1} \mathcal{D} A \mathcal{D}|D|^{-s-2-n}\right) \\
& \quad \sim_{c} \sum_{k}^{\prime} \sum_{l_{1}, l_{2}} a_{\alpha_{2}, l_{2}} a_{\alpha_{1}, l_{1}} \tau\left(U_{-k} U_{l_{1}} U_{k} U_{l_{2}}\right) \frac{k_{\mu_{1}}\left(k+l_{1}\right)_{\mu_{2}}}{|k|^{s+2+n}} \operatorname{Tr}\left(\gamma^{\alpha, \mu}\right) .
\end{aligned}
$$


which gives

$$
f_{\varepsilon J} J J^{-1} \mathcal{D} A \mathcal{D}|D|^{-2-n}=a_{\alpha_{2}, 0} a_{\alpha_{1}, 0} \underset{s=0}{\operatorname{Res}}\left(\sum_{k}^{\prime} \frac{k_{\mu_{1}} k_{\mu_{2}}}{|k|^{s+2+n}}\right) \operatorname{Tr}\left(\gamma^{\alpha, \mu}\right) .
$$

Thus,

$$
\begin{aligned}
& \frac{1}{2} f \tilde{A} \mathcal{D} \tilde{A} \mathcal{D}|D|^{-2-n} \\
& \quad=\left(a_{\alpha_{2}, 0} a_{\alpha_{1}, 0}-\sum_{l} a_{\alpha_{2},-l} a_{\alpha_{1}, l}\right) \operatorname{Res}_{s=0}\left(\sum_{k}^{\prime} \frac{k_{\mu_{1}} k_{\mu_{2}}}{|k|^{s+2+n}}\right) \operatorname{Tr}\left(\gamma^{\alpha, \mu}\right) .
\end{aligned}
$$

With $\sum_{k}^{\prime} \frac{k_{\mu_{1}} k_{\mu_{2}}}{|k|^{s+2+n}}=\frac{\delta_{\mu_{1} \mu_{2}}}{n} Z_{n}(s+n)$ and $C_{n}:=\operatorname{Res}_{s=0} Z_{n}(s+n)=2 \pi^{n / 2} \Gamma(n / 2)^{-1}$ we obtain that

$$
\frac{1}{2} f \tilde{A} \mathcal{D} \tilde{A} \mathcal{D}|D|^{-2-n}=\left(a_{\alpha_{2}, 0} a_{\alpha_{1}, 0}-\sum_{l} a_{\alpha_{2},-l} a_{\alpha_{1}, l}\right) \frac{C_{n}}{n} \operatorname{Tr}\left(\gamma^{\alpha_{2}} \gamma^{\mu} \gamma^{\alpha_{1}} \gamma_{\mu}\right) .
$$

Since $\operatorname{Tr}\left(\gamma^{\alpha_{2}} \gamma^{\mu} \gamma^{\alpha_{1}} \gamma_{\mu}\right)=2^{m}(2-n) \delta^{\alpha_{2}, \alpha_{1}}$, we get

$$
\frac{1}{2} f \tilde{A} \mathcal{D} \tilde{A} \mathcal{D}|D|^{-2-n}=2^{m}\left(-a_{\alpha, 0} a_{0}^{\alpha}+\sum_{l} a_{\alpha,-l} a_{l}^{\alpha}\right) \frac{C_{n}(n-2)}{n} .
$$

Equation (5.16) now proves the lemma.

Lemma 5.11. If $\frac{1}{2 \pi} \Theta$ is diophantine, then for any $P \in \Psi_{1}(\mathcal{A})$ and $q \in \mathbb{N}, q$ odd,

$$
f P|D|^{-(n-q)}=0 \text {. }
$$

Proof. There exist $B \in \mathcal{D}_{1}(\mathcal{A})$ and $p \in \mathbb{N}_{0}$ such that $P=B D^{-2 p}+R$ where $R$ is in $O P^{-q-1}$. Consequently, $f P|D|^{-(n-q)}=f B|D|^{-n-2 p+q}$. Assume that $B=a_{r} b_{r} \mathcal{D}^{q_{r-1}} a_{r-1} b_{r-1} \ldots \mathcal{D}^{q_{1}} a_{1} b_{1}$, where $r \in \mathbb{N}, a_{i} \in \mathcal{A}, b_{i} \in J \mathcal{A} J^{-1}, q_{i} \in \mathbb{N}$. If we prove that $f B|D|^{-n-2 p+q}=0$, then the general case will follow by linearity. Write $a_{i}=: \sum_{l} a_{i, l} U_{l}$ and $b_{i}=: \sum_{l} b_{i, l} U_{l}$. With $k_{\mu_{1}, \mu_{q_{i}}}:=k_{\mu_{1}} \ldots k_{\mu_{q_{i}}}$ and $\gamma^{\mu_{1}, \mu_{q_{i}}}=\gamma^{\mu_{1}} \ldots \gamma^{\mu q_{i}}$, we get

$$
\mathcal{D}^{q_{1}} a_{1} b_{1} U_{k} \otimes e_{j}=\sum_{l_{1}, l_{1}^{\prime}} a_{1, l_{1}} b_{1, l_{1}^{\prime}} U_{l_{1}} U_{k} U_{l_{1}^{\prime}}\left(k+l_{1}+l_{1}^{\prime}\right)_{\mu_{1}, \mu_{q_{1}}} \otimes \gamma^{\mu_{1}, \mu_{q_{1}}} e_{j},
$$

which gives after iteration

$$
\begin{aligned}
& B U_{k} \otimes e_{j}=\sum_{l, l^{\prime}} \tilde{a}_{l} \tilde{b}_{l} U_{l_{r}} \ldots U_{l_{1}} U_{k} U_{l_{1}^{\prime}} \ldots U_{l_{r}^{\prime}}
\end{aligned}
$$

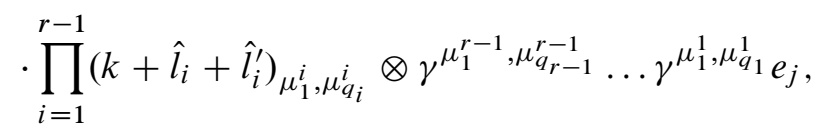


where $\tilde{a}_{l}:=a_{1, l_{1}} \ldots a_{r, l_{r}}$ and $\tilde{b}_{l^{\prime}}:=b_{1, l_{1}^{\prime}} \ldots b_{r, l_{r}^{\prime}}$. We denote $Q_{\mu}\left(k, l, l^{\prime}\right):=$

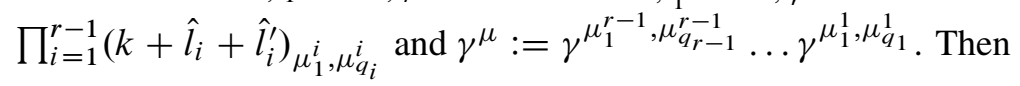

$$
\begin{aligned}
f B|D|^{-n-2 p+q}= & \operatorname{Res}_{s=0} \sum_{k}^{\prime} \sum_{l, l^{\prime}} \tilde{a}_{l} \tilde{b}_{l^{\prime}} \tau\left(U_{-k} U_{l_{r}} \ldots U_{l_{1}} U_{k} U_{l_{1}^{\prime}} \ldots U_{l_{r}^{\prime}}\right) \\
& \cdot \frac{Q_{\mu}\left(k, l, l^{\prime}\right)}{|k|^{s+2 p+n-q}} \operatorname{Tr}\left(\gamma^{\mu}\right) .
\end{aligned}
$$

Since $U_{l_{r}} \ldots U_{l_{1}} U_{k}=U_{k} U_{l_{r}} \ldots U_{l_{1}} e^{-i \sum_{1}^{r} l_{i} \cdot \Theta k}$, we get

$$
\tau\left(U_{-k} U_{l_{r}} \ldots U_{l_{1}} U_{k} U_{l_{1}^{\prime}} \ldots U_{l_{r}^{\prime}}\right)=\delta_{\sum_{1}^{r} l_{i}+l_{i}^{\prime}, 0} e^{i \phi\left(l, l^{\prime}\right)} e^{-i \sum_{1}^{r} l_{i} \cdot \Theta k}
$$

where $\phi$ is a real valued function. Thus,

$$
\begin{aligned}
& f B|D|^{-n-2 p+q} \\
& =\operatorname{Res}_{s=0} \sum_{k}^{\prime} \sum_{l, l^{\prime}} e^{i \phi\left(l, l^{\prime}\right)} \delta_{\sum_{1}^{r} l_{i}+l_{i}^{\prime}, 0} \tilde{a}_{l} \tilde{b}_{l^{\prime}} \frac{Q_{\mu}\left(k, l, l^{\prime}\right) e^{-i \sum_{1}^{r} l_{i} \cdot \Theta k}}{|k|^{s+2 p+n-q}} \operatorname{Tr}\left(\gamma^{\mu}\right) \\
& =: \operatorname{Res}_{s=0} f_{\mu}(s) \operatorname{Tr}\left(\gamma^{\mu}\right) .
\end{aligned}
$$

We decompose $Q_{\mu}\left(k, l, l^{\prime}\right)$ as a sum $\sum_{h=0}^{r} M_{h, \mu}\left(l, l^{\prime}\right) Q_{h, \mu}(k)$ where $Q_{h, \mu}$ is a homogeneous polynomial in $\left(k_{1}, \ldots, k_{n}\right)$ and $M_{h, \mu}\left(l, l^{\prime}\right)$ is a polynomial in $\left(\left(l_{1}\right)_{1}, \ldots,\left(l_{r}\right)_{n},\left(l_{1}^{\prime}\right)_{1}, \ldots,\left(l_{r}^{\prime}\right)_{n}\right)$.

Similarly, we decompose $f_{\mu}(s)$ as $\sum_{h=0}^{r} f_{h, \mu}(s)$. Theorem 2.6 (ii) implies that $f_{h, \mu}(s)$ extends meromorphically to the whole complex plane $\mathbb{C}$ with only one possible pole for $s+2 p+n-q=n+d$, where $d:=\operatorname{deg} Q_{h, \mu}$. In other words, if $d+q-2 p \neq 0$, then $f_{h, \mu}(s)$ is holomorphic at $s=0$. Suppose now that $d+q-2 p=0$ (note that this implies that $d$ is odd since $q$ is odd by hypothesis). Then it follows from Theorem 2.6 (ii) that

$$
\operatorname{Res}_{s=0} f_{h, \mu}(s)=V \int_{u \in S^{n-1}} Q_{h, \mu}(u) d S(u),
$$

with $V:=\sum_{l, l^{\prime} \in Z} M_{h, \mu}\left(l, l^{\prime}\right) e^{i \phi\left(l, l^{\prime}\right)} \delta_{\sum_{1}^{r} l_{i}+l_{i}^{\prime}, 0} \tilde{a}_{l} \tilde{b}_{l^{\prime}}$ and $Z:=\left\{l, l^{\prime} \mid \sum_{i=1}^{r} l_{i}=0\right\}$. Since $d$ is odd, $Q_{h, \mu}(-u)=-Q_{h, \mu}(u)$ and $\int_{u \in S^{n-1}} Q_{h, \mu}(u) d S(u)=0$. Thus, $\operatorname{Res}_{s=0} f_{h, \mu}(s)=0$ in any case, which gives the result.

As we have seen, the crucial point of the preceding lemma is the decomposition of the numerator of the series $f_{\mu}(s)$ as polynomials in $k$. This is possible because we restrict our pseudodifferential operators to $\Psi_{1}(\mathcal{A})$. 
Proof of Proposition 5.5. The first equality follows from Proposition 4.9 and according to (2.14) we have

$$
f|D|^{-n}=\operatorname{Res}_{s=0} \operatorname{Tr}\left(|D|^{-s-n}\right)=2^{m} \operatorname{Res}_{s=0} Z_{n}(s+n)=\frac{2^{m+1} \pi^{n / 2}}{\Gamma(n / 2)} .
$$

For the second equality, we get from Lemmas 5.7 and 4.6

$$
\operatorname{Res}_{s=n-k} \zeta_{D_{A}}(s)=\sum_{p=1}^{k} \sum_{r_{1}, \ldots, r_{p}=0}^{k-p} h(n-k, r, p) f \varepsilon^{r_{1}}(Y) \ldots \varepsilon^{r_{p}}(Y)|D|^{-(n-k)} .
$$

Corollary 4.4 and Lemma 5.11 imply that $f \varepsilon^{r_{1}}(Y) \ldots \varepsilon^{r_{p}}(Y)|D|^{-(n-k)}=0$, which gives the result.

The last equality follows from Lemma 5.10 and Corollary 4.11.

\section{The spectral action}

Here is the main result of this section.

Theorem 6.1. Consider the $n$-NC-torus $\left(C^{\infty}\left(\mathbb{T}_{\Theta}^{n}\right), \mathcal{H}, \mathcal{D}\right)$, where $n \in \mathbb{N}$ and $\frac{1}{2 \pi} \Theta$ is a real $n \times n$ skew-symmetric diophantine matrix, and a selfadjoint 1-form $A=$ $L\left(-i A_{\alpha}\right) \otimes \gamma^{\alpha}$. Then the full spectral action of $\mathcal{D}_{A}=\mathcal{D}+A+\epsilon J A J^{-1}$ is

(i) $\mathcal{S}\left(\mathcal{D}_{A}, \Phi, \Lambda\right)=4 \pi \Phi_{2} \Lambda^{2}+\mathcal{O}\left(\Lambda^{-2}\right)$ for $n=2$,

(ii) $\mathcal{S}\left(\mathcal{D}_{A}, \Phi, \Lambda\right)=8 \pi^{2} \Phi_{4} \Lambda^{4}-\frac{4 \pi^{2}}{3} \Phi(0) \tau\left(F_{\mu \nu} F^{\mu v}\right)+\mathcal{O}\left(\Lambda^{-2}\right)$ for $n=4$.

(iii) More generally, in $\mathcal{S}\left(\mathcal{D}_{A}, \Phi, \Lambda\right)=\sum_{k=0}^{n} \Phi_{n-k} c_{n-k}(A) \Lambda^{n-k}+\mathcal{O}\left(\Lambda^{-1}\right)$ we have $c_{n-2}(A)=0$ and $c_{n-k}(A)=0$ for $k$ odd. In particular, $c_{0}(A)=0$ when $n$ is odd.

This result (for $n=4$ ) has also been obtained in [20] using the heat kernel method. It is however of interest to obtain it via direct computations of (1.5) since it shows how efficient this formula is. As we will see, the computation of all the noncommutative integrals requires a lot of technical steps. One of the main points, namely to isolate where the Diophantine condition on $\Theta$ comes into play, is outlined here.

Remark 6.2. Note that all terms must be gauge invariants, that is, according to (5.9), they are invariant under $A_{\alpha} \rightarrow \gamma_{u}\left(A_{\alpha}\right)=u A_{\alpha} u^{*}+u \delta_{\alpha}\left(u^{*}\right)$. A special case is $u=U_{k}$ where $U_{k} \delta_{\alpha}\left(U_{k}^{*}\right)=-i k_{\alpha} U_{0}$.

In the same way, note that there is no contradiction to the commutative case where, for any selfadjoint 1-form $A, \mathcal{D}_{A}=\mathcal{D}$ (so $A$ is equivalent to 0 !), since we assume in Theorem 6.1 that $\Theta$ is diophantine, so $\mathcal{A}$ cannot be commutative. 
Conjecture 6.3. The constant term of the spectral action of $\mathcal{D}_{A}$ on the noncommutative $n$-torus is proportional to the constant term of the spectral action of $\mathcal{D}+A$ on the commutative $n$-torus.

Remark 6.4. The appearance of a Diophantine condition for $\Theta$ has been characterized in dimension 2 by Connes [6], Prop. 49, where in this case, $\Theta=\theta\left(\begin{array}{cc}0 & 1 \\ -1 & 0\end{array}\right)$ with $\theta \in \mathbb{R}$. In fact, the Hochschild cohomology $H\left(\mathcal{A}_{\Theta}, \mathcal{A}_{\Theta}{ }^{*}\right)$ satisfies $\operatorname{dim} H^{j}\left(\mathcal{A}_{\Theta}, \mathcal{A}_{\Theta}{ }^{*}\right)=2$ (or 1) for $j=1$ (or $j=2$ ) if and only if the irrational number $\theta$ satisfies a Diophantine condition like $\left|1-e^{i 2 \pi n \theta}\right|^{-1}=\mathcal{O}\left(n^{k}\right)$ for some $k$.

Recall that when the matrix $\Theta$ is quite irrational (see [23], Cor. 2.12), then the $\mathrm{C}^{*}$-algebra generated by $\mathcal{A}_{\Theta}$ is simple.

Remark 6.5. One can generalize the above theorem to the case $\mathcal{D}=-i g^{\mu}{ }_{\nu} \delta_{\mu} \otimes \gamma^{\nu}$ instead of (5.4) when $g$ is a positive definite constant matrix. The formulae in Theorem 6.1 are still valid, up to obvious modifications due to volume variation.

6.1. Computations of $f$. In order to get this theorem, let us prove a few technical lemmas.

We suppose from now on that $\Theta$ is a skew-symmetric matrix in $\mathcal{M}_{n}(\mathbb{R})$. No other hypothesis is assumed for $\Theta$, except when it is explicitly stated.

When $A$ is a selfadjoint 1 -form, we define for $n \in N, q \in \mathbb{N}, 2 \leq q \leq n$ and $\sigma \in\{-,+\}^{q}$ :

$$
\begin{aligned}
& \mathbb{A}^{+}:=A \mathcal{D} D^{-2}, \\
& \mathbb{A}^{-}:=\epsilon J A J^{-1} \mathcal{D} D^{-2}, \\
& \mathbb{A}^{\sigma}:=\mathbb{A}^{\sigma_{q}} \ldots \mathbb{A}^{\sigma_{1}} .
\end{aligned}
$$

Lemma 6.6. For any $q \in \mathbb{N}$ we have

$$
f\left(\tilde{A} D^{-1}\right)^{q}=f\left(\tilde{A} \mathcal{D} D^{-2}\right)^{q}=\sum_{\sigma \in\{+,-\}^{q}} f_{\mathbb{A}^{\sigma}} .
$$

Proof. Since $P_{0}$ belongs to $O P^{-\infty}$, it follows that $D^{-1}=\mathcal{D} D^{-2} \bmod O P^{-\infty}$ and $f\left(\tilde{A} D^{-1}\right)^{q}=f\left(\tilde{A} \mathcal{D} D^{-2}\right)^{q}$.

Lemma 6.7. Let $A$ be a selfadjoint 1-form, $n \in \mathbb{N}, q \in \mathbb{N}$ with $2 \leq q \leq n$, and $\sigma \in\{-,+\}^{q}$. Then

$$
f \mathbb{A}^{\sigma}=f \mathbb{A}^{-\sigma} \text {. }
$$

Proof. Let us first check that $J P_{0}=P_{0} J$. Since $\mathcal{D} J=\varepsilon J \mathcal{D}$, we get $\mathcal{D} J P_{0}=0$ and so $J P_{0}=P_{0} J P_{0}$. Since $J$ is an antiunitary operator, it follows that $P_{0} J=P_{0} J P_{0}$ 
and $P_{0} J=J P_{0}$. Consequently, $J D^{2}=D^{2} J, J \mathcal{D} D^{-2}=\varepsilon \mathcal{D} D^{-2} J, J \mathbb{A}^{+} J^{-1}=$ $\mathbb{A}^{-}$and $J \mathbb{A}^{-} J^{-1}=\mathbb{A}^{+}$. In summary, $J \mathbb{A}^{\sigma_{i}} J^{-1}=\mathbb{A}^{-\sigma_{i}}$.

The trace property of $f$ now gives

$$
f \mathbb{A}^{\sigma}=f \mathbb{A}^{\sigma_{q}} \ldots \mathbb{A}^{\sigma_{1}}=f J \mathbb{A}^{\sigma_{q}} J^{-1} \ldots \mathbb{A}^{\sigma_{1}} J^{-1} f \mathbb{A}^{-\sigma_{q}} \ldots \mathbb{A}^{-\sigma_{1}}=f \mathbb{A}^{-\sigma} .
$$

Definition 6.8. In [11] the vanishing tadpole hypothesis was introduced:

$$
f A D^{-1}=0 \text { for all } A \in \Omega_{\mathcal{D}}^{1}(\mathcal{A})
$$

By the following lemma, this condition is satisfied for the noncommutative torus, a fact more or less already known within the noncommutative community [35].

Lemma 6.9. Let $n \in \mathbb{N}$ and let $A=L\left(-i A_{\alpha}\right) \otimes \gamma^{\alpha}=-i \sum_{l \in \mathbb{Z}^{n}} a_{\alpha, l} U_{l} \otimes \gamma^{\alpha}$ be a hermitian 1-form with $A_{\alpha} \in \mathcal{A}_{\Theta},\left\{a_{\alpha, l}\right\}_{l} \in \mathcal{S}\left(\mathbb{Z}^{n}\right)$. Then

(i) $f A^{p} D^{-q}=f\left(\epsilon J A J^{-1}\right)^{p} D^{-q}=0$ for $p \geq 0$ and $1 \leq q<n$ (the case $p=q=1$ is the tadpole hypothesis);

(ii) if $\frac{1}{2 \pi} \Theta$ is diophantine, then $f B D^{-q}=0$ for $1 \leq q<n$ and any $B$ in the algebra generated by $\mathcal{A},[\mathcal{D}, \mathcal{A}], J \mathcal{A} J^{-1}$ and $J[\mathcal{D}, \mathcal{A}] J^{-1}$.

Proof. (i) Let us compute

$$
f A^{p}\left(\epsilon J A J^{-1}\right)^{p^{\prime}} D^{-q}
$$

With $A=L\left(-i A_{\alpha}\right) \otimes \gamma^{\alpha}$ and $\epsilon J A J^{-1}=R\left(i A_{\alpha}\right) \otimes \gamma^{\alpha}$, we get

$$
A^{p}=L\left(-i A_{\alpha_{1}}\right) \ldots L\left(-i A_{\alpha_{p}}\right) \otimes \gamma^{\alpha_{1}} \ldots \gamma^{\alpha_{p}}
$$

and

$$
\left(\epsilon J A J^{-1}\right)^{p^{\prime}}=R\left(i A_{\alpha_{1}^{\prime}}\right) \ldots R\left(i A_{\alpha_{p^{\prime}}^{\prime}}\right) \otimes \gamma^{\alpha_{1}^{\prime}} \ldots \gamma^{\alpha_{p^{\prime}}^{\prime}}
$$

Write $\tilde{a}_{\alpha, l}:=a_{\alpha_{1}, l_{1}} \ldots a_{\alpha_{p}, l_{p}}$. Since

$$
\begin{aligned}
& L\left(-i A_{\alpha_{1}}\right) \ldots L\left(-i A_{\alpha_{p}}\right) R\left(i A_{\alpha_{1}^{\prime}}\right) \ldots R\left(i A_{\alpha_{p^{\prime}}^{\prime}}\right) U_{k} \\
& \quad=(-i)^{p} i^{p^{\prime}} \sum_{l, l^{\prime}} \tilde{a}_{\alpha, l} \tilde{a}_{\alpha^{\prime}, l^{\prime}} U_{l_{1}} \ldots U_{l_{p}} U_{k} U_{l_{p^{\prime}}^{\prime}} \ldots U_{l_{1}^{\prime}},
\end{aligned}
$$


and

$$
U_{l_{1}} \ldots U_{l_{p}} U_{k}=U_{k} U_{l_{1}} \ldots U_{l_{p}} e^{-i\left(\sum_{i} l_{i}\right) \cdot \Theta k}
$$

we get

$$
\begin{aligned}
& A^{p}\left(\epsilon J A J^{-1}\right)^{p^{\prime}} D^{-q}|D|^{-s} U_{k} \otimes e_{i} \\
& \quad \sim_{c}(-i)^{p} i^{p^{\prime}} \sum_{l, l^{\prime}} g_{\mu, \alpha, \alpha^{\prime}}\left(s, k, l, l^{\prime}\right) U_{k} U_{l, l^{\prime}} \otimes \gamma^{\alpha, \alpha^{\prime}, \mu} e_{i},
\end{aligned}
$$

where we use the abbreviations $U_{l, l^{\prime}}:=U_{l_{1}} \ldots U_{l_{p}} U_{l_{p^{\prime}}} \ldots U_{l_{1}^{\prime}} g_{\mu, \alpha, \alpha^{\prime}}\left(s, k, l, l^{\prime}\right):=$ $e^{i k \cdot \Theta \sum_{j} l_{j}} \frac{k_{\mu_{1}} \ldots k_{\mu_{q}}}{|k|^{s+2 q}} \tilde{a}_{\alpha, l} \tilde{a}_{\alpha^{\prime}, l^{\prime}}$ and $\gamma^{\alpha, \alpha^{\prime}, \mu}:=\gamma^{\alpha_{1}} \ldots \gamma^{\alpha_{p}} \gamma^{\alpha_{1}^{\prime}} \ldots \gamma^{\alpha_{p^{\prime}}^{\prime}} \gamma^{\mu_{1}} \ldots \gamma^{\mu_{q}}$.

Thus, $f A^{p}\left(\epsilon J A J^{-1}\right)^{p^{\prime}} D^{-q}=\operatorname{Res}_{s=0} f(s)$ where

$$
\begin{aligned}
f(s): & =\operatorname{Tr}\left(A^{p}\left(\epsilon J A J^{-1}\right)^{p^{\prime}} D^{-q}|D|^{-s}\right) \\
& \sim_{c}(-i)^{p} i^{p^{\prime}} \sum_{k \in \mathbb{Z}^{n}}^{\prime}\left\langle U_{k} \otimes e_{i}, \sum_{l, l^{\prime}} g_{\mu, \alpha, \alpha^{\prime}}\left(s, k, l, l^{\prime}\right) U_{k} U_{l, l^{\prime}} \otimes \gamma^{\alpha, \alpha^{\prime}, \mu} e_{i}\right\rangle \\
& \sim_{c}(-i)^{p} i^{p^{\prime}} \sum_{k \in \mathbb{Z}^{n}}^{\prime} \tau\left(\sum_{l, l^{\prime}} g_{\mu, \alpha, \alpha^{\prime}}\left(s, k, l, l^{\prime}\right) U_{l, l^{\prime}}\right) \operatorname{Tr}\left(\gamma^{\mu, \alpha, \alpha^{\prime}}\right) \\
& \sim_{c}(-i)^{p} i^{p^{\prime}} \sum_{k \in \mathbb{Z}^{n}}^{\prime} \sum_{l, l^{\prime}} g_{\mu, \alpha, \alpha^{\prime}}\left(s, k, l, l^{\prime}\right) \tau\left(U_{l, l^{\prime}}\right) \operatorname{Tr}\left(\gamma^{\mu, \alpha, \alpha^{\prime}}\right) .
\end{aligned}
$$

It is straightforward to check that the series $\sum_{k, l, l^{\prime}}^{\prime} g_{\mu, \alpha, \alpha^{\prime}}\left(s, k, l, l^{\prime}\right) \tau\left(U_{l, l^{\prime}}\right)$ is absolutely summable if $\operatorname{Re}(s)>R$ for a $R>0$. Thus, we can exchange the summation over $k$ and $l, l^{\prime}$, which gives

$$
f(s) \sim_{c}(-i)^{p} i^{p^{\prime}} \sum_{l, l^{\prime}} \sum_{k \in \mathbb{Z}^{n}}^{\prime} g_{\mu, \alpha, \alpha^{\prime}}\left(s, k, l, l^{\prime}\right) \tau\left(U_{l, l^{\prime}}\right) \operatorname{Tr}\left(\gamma^{\mu, \alpha, \alpha^{\prime}}\right) .
$$

If we suppose now that $p^{\prime}=0$, we see that

$$
f(s) \sim_{c}(-i)^{p} \sum_{l} \sum_{k \in \mathbb{Z}^{n}} \frac{k_{\mu_{1}} \ldots k_{\mu_{q}}}{|k|^{s+2 q}} \tilde{a}_{\alpha, l} \delta_{\sum_{i=1}^{p} l_{i}, 0} \operatorname{Tr}\left(\gamma^{\mu, \alpha, \alpha^{\prime}}\right),
$$

which is, by Proposition 2.17, analytic at 0 . In particular, for $p=q=1$, we see that $f A D^{-1}=0$, i.e., the vanishing tadpole hypothesis is satisfied. Similarly, if we suppose that $p=0$, we get

$$
f(s) \sim_{c}(-i)^{p^{\prime}} \sum_{l^{\prime}} \sum_{k \in \mathbb{Z}^{n}} \frac{k_{\mu_{1}} \ldots k_{\mu_{q}}}{|k|^{s+2 q}} \tilde{a}_{\alpha, l^{\prime}} \delta_{\sum_{i=1}^{p^{\prime} l_{i}^{\prime}, 0}, 0} \operatorname{Tr}\left(\gamma^{\mu, \alpha, \alpha^{\prime}}\right)
$$

which is holomorphic at 0 . 
(ii) Adapting the proof of Lemma 5.11 to our setting (taking $q_{i}=0$ and adding gamma matrices components), we see that

$$
\begin{aligned}
& f B D^{-q} \\
& =\operatorname{Res}_{s=0} \sum_{k}^{\prime} \sum_{l, l^{\prime}} e^{i \phi\left(l, l^{\prime}\right)} \delta_{\sum_{1}^{r} l_{i}+l_{i}^{\prime}, 0} \tilde{a}_{\alpha, l} \tilde{b}_{\beta, l^{\prime}} \frac{k_{\mu_{1}} \ldots k_{\mu_{q}} e^{-i \sum_{1}^{r} l_{i} \cdot \Theta k}}{|k|^{s+2 q}} \operatorname{Tr}\left(\gamma^{(\mu, \alpha, \beta)}\right),
\end{aligned}
$$

where $\gamma^{(\mu, \alpha, \beta)}$ is a complicated product of gamma matrices. By Theorem 2.6(ii), since we suppose here that $\frac{1}{2 \pi} \Theta$ is diophantine, this residue is 0 .

\subsubsection{Even dimensional case}

Corollary 6.10. Let the same hypotheses be satisfied as in Lemma 6.9. Then:

(i) case $n=2: f A^{q} D^{-q}=-\delta_{q, 2} 4 \pi \tau\left(A_{\alpha} A^{\alpha}\right)$;

(ii) case $n=4$ :

$$
f A^{q} D^{-q}=\delta_{q, 4} \frac{\pi^{2}}{12} \tau\left(A_{\alpha_{1}} \ldots A_{\alpha_{4}}\right) \operatorname{Tr}\left(\gamma^{\alpha_{1}} \ldots \gamma^{\alpha_{4}} \gamma^{\mu_{1}} \ldots \gamma^{\mu_{4}}\right) \delta_{\mu_{1}, \ldots, \mu_{4}},
$$

where $\delta_{\mu_{1}, \ldots, \mu_{4}}:=\delta_{\mu_{1} \mu_{2}} \delta_{\mu_{3} \mu_{4}}+\delta_{\mu_{1} \mu_{3}} \delta_{\mu_{2} \mu_{4}}+\delta_{\mu_{1} \mu_{4}} \delta_{\mu_{2} \mu_{3}}$.

Proof. (i), (ii) The same computation as in Lemma 6.9(i) (with $p^{\prime}=0, p=q=n$ ) gives

$$
\begin{aligned}
f A^{n} D^{-n}= & \operatorname{Res}_{s=0}(-i)^{n}\left(\sum_{k \in \mathbb{Z}^{n}}^{\prime} \frac{k_{\mu_{1}} \ldots k_{\mu_{n}}}{|k|^{s+2 n}}\right) \\
& \cdot \tau\left(\sum_{l \in\left(\mathbb{Z}^{n}\right)^{n}} \tilde{a}_{\alpha, l} U_{l_{1}} \ldots U_{l_{n}}\right) \operatorname{Tr}\left(\gamma^{\alpha_{1}} \ldots \gamma^{\alpha_{n}} \gamma^{\mu_{1}} \ldots \gamma^{\mu_{n}}\right),
\end{aligned}
$$

and the result follows from Proposition 2.17.

We will need some notations:

Let $n \in \mathbb{N}, q \geq 2$. Let $l:=\left(l_{1}, \ldots, l_{q-1}\right) \in\left(\mathbb{Z}^{n}\right)^{q-1}, \alpha:=\left(\alpha_{1}, \ldots, \alpha_{q}\right) \in$ $\{1, \ldots, n\}^{q}, k \in \mathbb{Z}^{n} \backslash\{0\}, \sigma \in\{-,+\}^{q},\left(a_{i}\right)_{1 \leq i \leq n} \in\left(\mathcal{S}\left(\mathbb{Z}^{n}\right)\right)^{n}$, and let

$$
\begin{aligned}
& l_{q}:=-\sum_{1 \leq j \leq q-1} l_{j}, \quad \lambda_{\sigma}:=(-i)^{q} \prod_{j=1 \ldots q} \sigma_{j}, \quad \tilde{a}_{\alpha, l}:=a_{\alpha_{1}, l_{1}} \ldots a_{\alpha_{q}, l_{q}}, \\
& \phi_{\sigma}(k, l):=\sum_{1 \leq j \leq q-1}\left(\sigma_{j}-\sigma_{q}\right) k \cdot \Theta l_{j}+\sum_{2 \leq j \leq q-1} \sigma_{j}\left(l_{1}+\cdots+l_{j-1}\right) \cdot \Theta l_{j}, \\
& g_{\mu}(s, k, l):=\frac{k_{\mu_{1}}\left(k+l_{1}\right)_{\mu_{2}} \ldots\left(k+l_{1}+\cdots+l_{q-1}\right)_{\mu_{q}}}{|k|^{s+2}\left|k+l_{1}\right|^{2} \ldots\left|k+l_{1}+\cdots+l_{q-1}\right|^{2}} .
\end{aligned}
$$


Here we use the convention $\sum_{2 \leq j \leq q-1}=0$ when $q=2$, and $g_{\mu}(s, k, l)=0$ whenever $\hat{l}_{i}=-k$ for a $1 \leq i \leq q-1$.

Lemma 6.11. Let $A=L\left(-i A_{\alpha}\right) \otimes \gamma^{\alpha}=-i \sum_{l \in \mathbb{Z}^{n}} a_{\alpha, l} U_{l} \otimes \gamma^{\alpha}$ be a hermitian 1-form, where $A_{\alpha}=-A_{\alpha}^{*} \in \mathcal{A}_{\Theta}$ and $\left\{a_{\alpha, l}\right\}_{l} \in \mathcal{S}\left(\mathbb{Z}^{n}\right)$ with $n \in \mathbb{N}$, and let $2 \leq q \leq n$, $\sigma \in\{-,+\}^{q}$.

$$
\begin{aligned}
& \text { Then } f \mathbb{A}^{\sigma}=\operatorname{Res}_{s=0} f(s) \text { where } \\
& f(s):=\sum_{l \in\left(\mathbb{Z}^{n}\right)^{q-1}} \sum_{k \in \mathbb{Z}^{n}}^{\prime} \lambda_{\sigma} e^{\frac{i}{2} \phi_{\sigma}(k, l)} g_{\mu}(s, k, l) \tilde{a}_{\alpha, l} \operatorname{Tr}\left(\gamma^{\alpha_{q}} \gamma^{\mu_{q}} \ldots \gamma^{\alpha_{1}} \gamma^{\mu_{1}}\right) .
\end{aligned}
$$

Proof. By definition, $f \mathbb{A}^{\sigma}=\operatorname{Res}_{s=0} f(s)$ where

$$
\operatorname{Tr}\left(\mathbb{A}^{\sigma_{q}} \ldots \mathbb{A}^{\sigma_{1}}|D|^{-s}\right) \sim_{c} \sum_{k \in \mathbb{Z}^{n}}^{\prime}\left\langle U_{k} \otimes e^{i},|k|^{-s} \mathbb{A}^{\sigma_{q}} \ldots \mathbb{A}^{\sigma_{1}} U_{k} \otimes e_{i}\right\rangle=: f(s) .
$$

Let $r \in \mathbb{Z}^{n}$ and $v \in \mathbb{C}^{2^{m}}$. Since $A=L\left(-i A_{\alpha}\right) \otimes \gamma^{\alpha}$ and $\epsilon J A J^{-1}=R\left(i A_{\alpha}\right) \otimes \gamma^{\alpha}$, we get

$$
\begin{aligned}
\mathbb{A}^{+} U_{r} \otimes v & =A \mathcal{D} D^{-2} U_{r} \otimes v \\
& =A \frac{r_{\mu}}{|r|^{2}+\delta_{r, 0}} U_{r} \otimes \gamma^{\mu} v \\
& =-i \frac{r_{\mu}}{|r|^{2}+\delta_{r, 0}} A_{\alpha} U_{r} \otimes \gamma^{\alpha} \gamma^{\mu} v, \\
\mathbb{A}^{-} U_{r} \otimes v & =\epsilon J J^{-1} \mathcal{D} D^{-2} U_{r} \otimes v \\
& =\epsilon J A J^{-1} \frac{r_{\mu}}{|r|^{2}+\delta_{r, 0}} U_{r} \otimes \gamma^{\mu} v \\
& =i \frac{r_{\mu}}{|r|^{2}+\delta_{r, 0}} U_{r} A_{\alpha} \otimes \gamma^{\alpha} \gamma^{\mu} v .
\end{aligned}
$$

With $U_{l} U_{r}=e^{\frac{i}{2} r \cdot \Theta l} U_{r+l}$ and $U_{r} U_{l}=e^{-\frac{i}{2} r \cdot \Theta l} U_{r+l}$ we obtain that

$$
\mathbb{A}^{\sigma_{j}} U_{r} \otimes v=\sum_{l \in \mathbb{Z}^{n}}\left(-\sigma_{j}\right) i e^{\sigma_{j} \frac{i}{2} r \cdot \Theta l} \frac{r_{\mu}}{|r|^{2}+\delta_{r, 0}} a_{\alpha, l} U_{r+l} \otimes \gamma^{\alpha} \gamma^{\mu} v .
$$

for any $1 \leq j \leq q$. We now apply $q$ times this formula to get

$$
\begin{aligned}
& |k|^{-s} \mathbb{A}^{\sigma_{q}} \ldots \mathbb{A}^{\sigma_{1}} U_{k} \otimes e_{i} \\
& \quad=\lambda_{\sigma} \sum_{l \in\left(\mathbb{Z}^{n}\right)^{q}} e^{\frac{i}{2} \phi_{\sigma}(k, l)} g_{\mu}(s, k, l) \tilde{a}_{\alpha, l} U_{k+\sum_{j} l_{j}} \otimes \gamma^{\alpha_{q}} \gamma^{\mu_{q}} \ldots \gamma^{\alpha_{1}} \gamma^{\mu_{1}} e_{i}
\end{aligned}
$$


with

$$
\phi_{\sigma}(k, l):=\sigma_{1} k \cdot \Theta l_{1}+\sigma_{2}\left(k+l_{1}\right) \cdot \Theta l_{2}+\cdots+\sigma_{q}\left(k+l_{1}+\cdots+l_{q-1}\right) \cdot \Theta l_{q} .
$$

Thus

$$
\begin{aligned}
f(s) & =\sum_{k \in \mathbb{Z}^{n}}^{\prime} \tau\left(\lambda_{\sigma} \sum_{l \in\left(\mathbb{Z}^{n}\right)^{q}} e^{\frac{i}{2} \phi_{\sigma}(k, l)} g_{\mu}(s, k, l) \tilde{a}_{\alpha, l} U_{\sum_{j} l_{j}} e^{\frac{i}{2} k \cdot \Theta \sum_{j} l_{j}}\right) \\
& \operatorname{Tr}\left(\gamma^{\alpha_{q}} \gamma^{\mu_{q}} \ldots \gamma^{\alpha_{1}} \gamma^{\mu_{1}}\right) \\
& =\sum_{k \in \mathbb{Z}^{n}}^{\prime} \lambda_{\sigma} \sum_{l \in\left(\mathbb{Z}^{n}\right)^{q}} e^{\frac{i}{2} \phi_{\sigma}(k, l)} g_{\mu}(s, k, l) \tilde{a}_{\alpha, l} \delta\left(\sum_{j} l_{j}\right) \operatorname{Tr}\left(\gamma^{\alpha_{q}} \gamma^{\mu_{q}} \ldots \gamma^{\alpha_{1}} \gamma^{\mu_{1}}\right) \\
& =\sum_{k \in \mathbb{Z}^{n}}^{\prime} \lambda_{\sigma} \sum_{l \in\left(\mathbb{Z}^{n}\right)^{q-1}} e^{\frac{i}{2} \phi_{\sigma}(k, l)} g_{\mu}(s, k, l) \tilde{a}_{\alpha, l} \operatorname{Tr}\left(\gamma^{\alpha_{q}} \gamma^{\mu_{q}} \ldots \gamma^{\alpha_{1}} \gamma^{\mu_{1}}\right),
\end{aligned}
$$

where in the last sum $l_{q}$ is fixed to $-\sum_{1 \leq j \leq q-1} l_{j}$. Hence

$$
\phi_{\sigma}(k, l)=\sum_{1 \leq j \leq q-1}\left(\sigma_{j}-\sigma_{q}\right) k \cdot \Theta l_{j}+\sum_{2 \leq j \leq q-1} \sigma_{j}\left(l_{1}+\cdots+l_{j-1}\right) \cdot \Theta l_{j} .
$$

By Lemma 2.11 there exists $R>0$ such that for any $s \in \mathbb{C}$ with $\operatorname{Re}(s)>R$ the family

$$
\left(e^{\frac{i}{2} \phi_{\sigma}(k, l)} g_{\mu}(s, k, l) \tilde{a}_{\alpha, l}\right)_{(k, l) \in\left(\mathbb{Z}^{n} \backslash\{0\}\right) \times\left(\mathbb{Z}^{n}\right)^{q-1}}
$$

is absolutely summable as a linear combination of families of the type considered in that lemma. As a consequence, we can exchange the summations over $k$ and $l$, which gives the result.

In the following we will use the abbreviation

$$
c:=\frac{4 \pi^{2}}{3}
$$

Lemma 6.12. Suppose that $n=4$ and let the same hypotheses be satisfied as in Lemma 6.11. Then:

$$
\begin{aligned}
& \text { (i) } \frac{1}{2} f\left(\mathbb{A}^{+}\right)^{2}=\frac{1}{2} f\left(\mathbb{A}^{-}\right)^{2}=c \sum_{l \in \mathbb{Z}^{4}} a_{\alpha_{1}, l} a_{\alpha_{2},-l}\left(l^{\alpha_{1}} l^{\alpha_{2}}-\delta^{\alpha_{1} \alpha_{2}}|l|^{2}\right) . \\
& \text { (ii) }-\frac{1}{3} f\left(\mathbb{A}^{+}\right)^{3}=-\frac{1}{3} f\left(\mathbb{A}^{-}\right)^{3}=4 c \sum_{l_{i} \in \mathbb{Z}^{4}} a_{\alpha_{3},-l_{1}-l_{2}} a_{l_{2}}^{\alpha_{1}} a_{\alpha_{1}, l_{1}} \sin \frac{l_{1} \cdot \Theta l_{2}}{2} l_{1}^{\alpha_{3}} . \\
& \text { (iii) } \frac{1}{4} f\left(\mathbb{A}^{+}\right)^{4}=\frac{1}{4} f\left(\mathbb{A}^{-}\right)^{4} \\
& \quad=2 c \sum_{l_{i} \in \mathbb{Z}^{4}} a_{\alpha_{1},-l_{1}-l_{2}-l_{3}} a_{\alpha_{2}, l_{3}} a_{l_{2}}^{\alpha_{1}} a_{l_{1}}^{\alpha_{2}} \sin \frac{l_{1} \cdot \Theta\left(l_{2}+l_{3}\right)}{2} \sin \frac{l_{2} \cdot \Theta l_{3}}{2} .
\end{aligned}
$$


(iv) Suppose that $\frac{1}{2 \pi} \Theta$ is diophantine. Then the crossed terms in $f\left(\mathbb{A}^{+}+\mathbb{A}^{-}\right)^{q}$ vanish: if $C$ is the set of all $\sigma \in\{-,+\}^{q}$ with $2 \leq q \leq 4$ such that there exist $i, j$ satisfying $\sigma_{i} \neq \sigma_{j}$, then we have $\sum_{\sigma \in C} f \mathbb{A}^{\sigma}=0$.

Proof. (i) Lemma 6.11 implies that $f \mathbb{A}^{++}=\operatorname{Res}_{s=0} \sum_{l \in \mathbb{Z}^{n}}-f(s, l)$ where

$$
f(s, l):=\sum_{k \in \mathbb{Z}^{n}}^{\prime} \frac{k_{\mu_{1}}(k+l)_{\mu_{2}}}{|k|^{s+2}|k+l|^{2}} \tilde{a}_{\alpha, l} \operatorname{Tr}\left(\gamma^{\alpha_{2}} \gamma^{\mu_{2}} \gamma^{\alpha_{1}} \gamma^{\mu_{1}}\right) \quad \text { and } \quad \tilde{a}_{\alpha, l}:=a_{\alpha_{1}, l} a_{\alpha_{2},-l} .
$$

We will now reduce the computation of the residue of an expression involving terms like $|k+l|^{2}$ in the denominator to the computation of residues of zeta functions. To proceed, we use (2.10) in an expression like the one appearing in $f(s, l)$. We see that the last term on the right-hand side yields a $Z_{n}(s)$, while the first one is less divergent by one power of $k$. If this is not enough, we repeat this operation for the new factor of $|k+l|^{2}$ in the denominator. For $f(s, l)$, which is quadratically divergent at $s=0$, we have to repeat this operation three times before ending with a convergent result. All the remaining terms are expressible in terms of $Z_{n}$ functions. We get, using three times (2.10),

$$
\frac{1}{|k+l|^{2}}=\frac{1}{|k|^{2}}-\frac{2 k \cdot l+|l|^{2}}{|k|^{4}}+\frac{\left(2 k \cdot l+|l|^{2}\right)^{2}}{|k|^{6}}-\frac{\left(2 k \cdot l+|l|^{2}\right)^{3}}{|k|^{6}|k+l|^{2}} .
$$

Put

$$
f_{\alpha, \mu}(s, l):=\sum_{k \in \mathbb{Z}^{n}}^{\prime} \frac{k_{\mu_{1}}(k+l)_{\mu_{2}}}{|k|^{s+2}|k+l|^{2}} \tilde{a}_{\alpha, l}
$$

so that $f(s, l)=f_{\alpha, \mu}(s, l) \operatorname{Tr}\left(\gamma^{\alpha_{2}} \gamma^{\mu_{2}} \gamma^{\alpha_{1}} \gamma^{\mu_{1}}\right)$. Equation (6.2) gives

$$
f_{\alpha, \mu}(s, l)=f_{1}(s, l)-f_{2}(s, l)+f_{3}(s, l)-r(s, l),
$$

with the obvious identifications. Note that the function

$$
r(s, l)=\sum_{k \in \mathbb{Z}^{n}}^{\prime} \frac{k_{\mu_{1}}(k+l)_{\mu_{2}}\left(2 k l+|l|^{2}\right)^{3}}{|k|^{s+8}|k+l|^{2}} \tilde{a}_{\alpha, l}
$$

is a linear combination of functions of the type $H(s, l)$ satisfying the hypothesis of Corollary 2.14. Thus $r(s, l)$ satisfies (H1), and with the previously seen equivalence relation modulo functions satisfying this hypothesis we get $f_{\alpha, \mu}(s, l) \sim f_{1}(s, l)-$ $f_{2}(s, l)+f_{3}(s, l)$.

We now compute $f_{1}(s, l)$ :

$$
f_{1}(s, l)=\sum_{k \in \mathbb{Z}^{n}}^{\prime} \frac{k_{\mu_{1}}(k+l) \mu_{\mu_{2}}}{|k|^{s+4}} \tilde{a}_{\alpha, l}=\tilde{a}_{\alpha, l} \sum_{k \in \mathbb{Z}^{n}}^{\prime} \frac{k_{\mu_{1}} k_{\mu_{2}}}{|k|^{s+4}}+0 .
$$


Proposition 2.1 gives that $s \mapsto \sum_{k \in \mathbb{Z}^{n}} \frac{k_{\mu_{1}} k_{\mu_{2}}}{|k|^{s+4}}$ is holomorphic at 0 . Thus $f_{1}(s, l)$ satisfies (H1) and $f_{\alpha, \mu}(s, l) \sim-f_{2}(s, l)+f_{3}(s, l)$.

Let us now compute $f_{2}(s, l)$ modulo (H1). Invoking several times Proposition 2.1 we obtain that

$$
\begin{aligned}
f_{2}(s, l) & =\sum_{k \in \mathbb{Z}^{n}}^{\prime} \frac{k_{\mu_{1}}(k+l)_{\mu_{2}}\left(2 k l+|l|^{2}\right)}{|k|^{s+6}} \tilde{a}_{\alpha, l} \\
& =\sum_{k \in \mathbb{Z}^{n}}^{\prime} \frac{(2 k l) k_{\mu_{1}} k_{\mu_{2}}+(2 k l) k_{\mu_{1}} l_{\mu_{2}}+|l|^{2} k_{\mu_{1}} k_{\mu_{2}}+l_{\mu_{2}}|l|^{2} k_{\mu_{1}}}{|k|^{s+6}} \tilde{a}_{\alpha, l} \\
& \sim 0+\sum_{k \in \mathbb{Z}^{n}}^{\prime} \frac{(2 k l) k_{\mu_{1}} l_{\mu_{2}}}{|k|^{s+6}} \tilde{a}_{\alpha, l}+\sum_{k \in \mathbb{Z}^{n}}^{\prime} \frac{|l|^{2} k_{\mu_{1}} k_{\mu_{2}}}{|k|^{s+6}} \tilde{a}_{\alpha, l}+0 .
\end{aligned}
$$

Recall that $\sum_{k \in \mathbb{Z}^{n}}^{\prime} \frac{k_{i} k_{j}}{|k|^{s+6}}=\frac{\delta_{i j}}{n} Z_{n}(s+4)$. Thus,

$$
f_{2}(s, l) \sim 2 l^{i} l_{\mu_{2}} \tilde{a}_{\alpha, l} \frac{\delta_{i \mu_{1}}}{n} Z_{n}(s+4)+|l|^{2} \tilde{a}_{\alpha, l} \frac{\delta_{\mu_{1} \mu_{2}}}{n} Z_{n}(s+4) .
$$

We compute $f_{3}(s, l)$ modulo (H1) following the same principles:

$$
\begin{aligned}
f_{3}(s, l)= & \sum_{k \in \mathbb{Z}^{n}}^{\prime} \frac{k_{\mu_{1}}(k+l)_{\mu_{2}}\left(2 k l+|l|^{2}\right)^{2}}{|k|^{s+8}} \tilde{a}_{\alpha, l} \\
= & \sum_{k \in \mathbb{Z}^{n}}^{\prime} \frac{(2 k l)^{2} k_{\mu_{1}} k_{\mu_{2}}+(2 k l)^{2} k_{\mu_{1}} l_{\mu_{2}}+|l|^{4} k_{\mu_{1}} k_{\mu_{2}}+|l|^{4} k_{\mu_{1}} l_{\mu_{2}}}{|k|^{s+8}} \\
& +\frac{(4 k l)|l|^{2} k_{\mu_{1}} k_{\mu_{2}}+(4 k l)|l|^{2} k_{\mu_{1}} l_{\mu_{2}}}{|k|^{s+8}} \tilde{a}_{\alpha, l} \\
\sim & 4 l^{i} l^{j} \sum_{k \in \mathbb{Z}^{n}}^{\prime} \frac{k_{i} k_{j} k_{\mu_{1}} k_{\mu_{2}}}{|k|^{s+8}} \tilde{a}_{\alpha, l}+0 .
\end{aligned}
$$

Finally we have

$$
\begin{aligned}
& f_{\alpha, \mu}(s, l) \\
& \quad \sim-\frac{1}{4}\left(2 l_{\mu_{1}} l_{\mu_{2}}+|l|^{2} \delta_{\mu_{1} \mu_{2}}\right) \tilde{a}_{\alpha, l} Z_{n}(s+4)+4 l^{i} l^{j} \tilde{a}_{\alpha, l} \sum_{k \in \mathbb{Z}^{n}}^{\prime} \frac{k_{i} k_{j} k_{\mu_{1}} k_{\mu_{2}}}{|k|^{s+8}} \\
& \quad=: g_{\alpha, \mu}(s, l) .
\end{aligned}
$$

Proposition 2.1 implies that $Z_{n}(s+4)$ and $s \mapsto \sum_{k \in \mathbb{Z}^{n}}^{\prime} \frac{k_{i} k_{j} k_{\mu_{1}} k_{\mu_{2}}}{|k|^{s+8}}$ extend holomorphically in a punctured open disk centered at 0 . Thus, $g_{\alpha, \mu}(s, l)$ satisfies $(\mathrm{H} 2)$ and 
we can apply Lemma 2.15 to get

$$
\begin{aligned}
-f\left(\mathbb{A}^{+}\right)^{2} & =\operatorname{Res}_{s=0} \sum_{l \in \mathbb{Z}^{n}} f(s, l) \\
& =\sum_{l \in \mathbb{Z}^{n}} \operatorname{Res}_{s=0} g_{\alpha, \mu}(s, l) \operatorname{Tr}\left(\gamma^{\alpha_{2}} \gamma^{\mu_{2}} \gamma^{\alpha_{1}} \gamma^{\mu_{1}}\right)=: \sum_{l \in \mathbb{Z}^{n}} \operatorname{Res}_{s=0} g(s, l) .
\end{aligned}
$$

In this way the problem is now reduced to the computation of $\operatorname{Res}_{s=0} g(s, l)$. Recall that $\operatorname{Res}_{s=0} Z_{4}(s+4)=2 \pi^{2}$ by (2.14) or (2.11) and

$$
\operatorname{Res}_{s=0} \sum_{k \in \mathbb{Z}^{n}}^{\prime} \frac{k_{i} k_{j} k_{l} k_{m}}{|k|^{s+8}}=\left(\delta_{i j} \delta_{l m}+\delta_{i l} \delta_{j m}+\delta_{i m} \delta_{j l}\right) \frac{\pi^{2}}{12}
$$

Thus,

$$
\operatorname{Res}_{s=0} g_{\alpha, \mu}(s, l)=-\frac{\pi^{2}}{3} \tilde{a}_{\alpha, l}\left(l_{\mu_{1}} l_{\mu_{2}}+\frac{1}{2}|l|^{2} \delta_{\mu_{1} \mu_{2}}\right) \text {. }
$$

We will use

$$
\operatorname{Tr}\left(\gamma^{\mu_{1}} \ldots \gamma^{\mu_{2 j}}\right)=\operatorname{Tr}(1) \sum_{\text {all pairings of }\{1 \ldots 2 j\}} s(P) \delta_{\mu_{P_{1}} \mu_{P_{2}}} \delta_{\mu_{P_{3}} \mu_{P_{4}}} \ldots \delta_{\mu_{P_{2 j}-1} \mu_{P_{2 j}}}
$$

where $s(P)$ is the signature of the permutation $P$ when $P_{2 m-1}<P_{2 m}$ for $1 \leq m \leq n$. This gives

$$
\operatorname{Tr}\left(\gamma^{\alpha_{2}} \gamma^{\mu_{2}} \gamma^{\alpha_{1}} \gamma^{\mu_{1}}\right)=2^{m}\left(\delta^{\alpha_{2} \mu_{2}} \delta^{\alpha_{1} \mu_{1}}-\delta^{\alpha_{1} \alpha_{2}} \delta^{\mu_{2} \mu_{1}}+\delta^{\alpha_{2} \mu_{1}} \delta^{\mu_{2} \alpha_{1}}\right) .
$$

Hence

$$
\begin{aligned}
& \underset{s=0}{\operatorname{Res}} g(s, l) \\
& \quad=-c \tilde{a}_{\alpha, l}\left(l_{\mu_{1}} l_{\mu_{2}}+\frac{1}{2}|l|^{2} \delta_{\mu_{1} \mu_{2}}\right)\left(\delta^{\alpha_{2} \mu_{2}} \delta^{\alpha_{1} \mu_{1}}-\delta^{\alpha_{1} \alpha_{2}} \delta^{\mu_{2} \mu_{1}}+\delta^{\alpha_{2} \mu_{1}} \delta^{\mu_{2} \alpha_{1}}\right) \\
& \quad=-2 c \tilde{a}_{\alpha, l}\left(l^{\alpha_{1}} l^{\alpha_{2}}-\delta^{\alpha_{1} \alpha_{2}}|l|^{2}\right) .
\end{aligned}
$$

Finally,

$$
\frac{1}{2} f\left(\mathbb{A}^{+}\right)^{2}=\frac{1}{2} f\left(\mathbb{A}^{-}\right)^{2}=c \sum_{l \in \mathbb{Z}^{n}} a_{\alpha_{1}, l} a_{\alpha_{2},-l}\left(l^{\alpha_{1}} l^{\alpha_{2}}-\delta^{\alpha_{1} \alpha_{2}}|l|^{2}\right) .
$$

(ii) By Lemma 6.11 we have $f \mathbb{A}^{+++}=\operatorname{Res}_{s=0} \sum_{\left(l_{1}, l_{2}\right) \in\left(\mathbb{Z}^{n}\right)^{2}} f(s, l)$ where

$$
\begin{aligned}
f(s, l) & :=\sum_{k \in \mathbb{Z}^{n}}^{\prime} i e^{\frac{i}{2} l_{1} \Theta l_{2}} \frac{k_{\mu_{1}}\left(k+l_{1}\right)_{\mu_{2}}\left(k+\hat{l}_{2}\right)_{\mu_{3}}}{|k|^{s+2}\left|k+l_{1}\right|^{2}\left|k+\hat{l}_{2}\right|^{2}} \tilde{a}_{\alpha, l} \operatorname{Tr}\left(\gamma^{\alpha_{3}} \gamma^{\mu_{3}} \gamma^{\alpha_{2}} \gamma^{\mu_{2}} \gamma^{\alpha_{1}} \gamma^{\mu_{1}}\right) \\
& =: f_{\alpha, \mu}(s, l) \operatorname{Tr}\left(\gamma^{\alpha_{3}} \gamma^{\mu_{3}} \gamma^{\alpha_{2}} \gamma^{\mu_{2}} \gamma^{\alpha_{1}} \gamma^{\mu_{1}}\right)
\end{aligned}
$$


and $\tilde{a}_{\alpha, l}:=a_{\alpha_{1}, l_{1}} a_{\alpha_{2}, l_{2}} a_{\alpha_{3},-\hat{l}_{2}}$ with $\hat{l}_{2}:=l_{1}+l_{2}$.

We use the same technique as in (i):

$$
\begin{aligned}
& \frac{1}{\left|k+l_{1}\right|^{2}}=\frac{1}{|k|^{2}}-\frac{2 k \cdot l_{1}+\left|l_{1}\right|^{2}}{|k|^{4}}+\frac{\left(2 k \cdot l_{1}+\left|l_{1}\right|^{2}\right)^{2}}{|k|^{4}\left|k+l_{1}\right|^{2}}, \\
& \frac{1}{\left|k+\hat{l}_{2}\right|^{2}}=\frac{1}{|k|^{2}}-\frac{2 k \cdot \hat{l}_{2}+\left|\hat{l}_{2}\right|^{2}}{|k|^{4}}+\frac{\left(2 k \cdot \hat{l}_{2}+\left|\hat{l}_{2}\right|^{2}\right)^{2}}{|k|^{4}\left|k+\hat{l}_{2}\right|^{2}}
\end{aligned}
$$

and thus

$$
\frac{1}{\left|k+l_{1}\right|^{2}\left|k+\hat{l}_{2}\right|^{2}}=\frac{1}{|k|^{4}}-\frac{2 k \cdot l_{1}}{|k|^{6}}-\frac{2 k \cdot \hat{l}_{2}}{|k|^{6}}+R(k, l),
$$

where the remain $R(k, l)$ is a term of order at most -6 in $k$. Equation (6.5) gives

$$
f_{\alpha, \mu}(s, l)=f_{1}(s, l)+r(s, l)
$$

where $r(s, l)$ corresponds to $R(k, l)$. Note that the function

$$
r(s, l)=\sum_{k \in \mathbb{Z}^{n}}^{\prime} i e^{\frac{i}{2} l_{1} \Theta l_{2}} \frac{k_{\mu_{1}}(k+l)_{\mu_{2}}\left(k+\hat{l}_{2}\right)_{\mu_{3}} R(k, l)}{|k|^{s+2}} \tilde{a}_{\alpha, l}
$$

is a linear combination of functions of the type $H(s, l)$ satisfying the hypothesis of Corollary 2.14. Thus, $r(s, l)$ satisfies $(\mathrm{H} 1)$ and $f_{\alpha, \mu}(s, l) \sim f_{1}(s, l)$.

Let us compute $f_{1}(s, l)$ modulo $(\mathrm{H} 1)$ :

$$
\begin{aligned}
f_{1}(s, l)= & \sum_{k \in \mathbb{Z}^{n}}^{\prime} i e^{\frac{i}{2} l_{1} \Theta l_{2}} \frac{k_{\mu_{1}}\left(k+l_{1}\right)_{\mu_{2}}\left(k+\hat{l}_{2}\right)_{\mu_{3}}}{|k|^{s+6}} \tilde{a}_{\alpha, l} \\
& \quad-\sum_{k \in \mathbb{Z}^{n}}^{\prime} i e^{\frac{i}{2} l_{1} \Theta l_{2}} \frac{k_{\mu_{1}}\left(k+l_{1}\right)_{\mu_{2}}\left(k+\hat{l}_{2}\right)_{\mu_{3}}\left(2 k \cdot l_{1}+2 k \cdot \hat{l}_{2}\right)}{|k|^{s+8}} \tilde{a}_{\alpha, l} \\
\sim & \sum_{k \in \mathbb{Z}^{n}}^{\prime} i e^{\frac{i}{2} l_{1} \Theta l_{2}} \frac{k_{\mu_{1}} k_{\mu_{2}} \widehat{l}_{2 \mu_{3}}+k_{\mu_{1}} k_{\mu_{3}} l_{1 \mu_{2}}}{|k|^{s+6}} \tilde{a}_{\alpha, l} \\
& \quad-\sum_{k \in \mathbb{Z}^{n}}^{\prime} i e^{\frac{i}{2} l_{1} \Theta l_{2}} \frac{k_{\mu_{1}} k_{\mu_{2}} k_{\mu_{3}}\left(2 k \cdot l_{1}+2 k \cdot \hat{l}_{2}\right)}{|k|^{s+8}} \tilde{a}_{\alpha, l} \\
= & i e^{\frac{i}{2} l_{1} \Theta l_{2}} \tilde{a}_{\alpha, l}\left(\left(l_{1} \mu_{2} \delta_{\mu_{1} \mu_{3}}+\widehat{l}_{2 \mu_{3}} \delta_{\mu_{1} \mu_{2}}\right) \frac{1}{4} Z_{4}(s+4)\right. \\
& \left.\quad-2\left(l_{1}^{i}+\hat{l}_{2}^{i}\right) \sum_{k \in \mathbb{Z}^{n}}^{\prime} \frac{k_{\mu_{1}} k_{\mu_{2}} k_{\mu_{3}} k_{i}}{|k|^{s+8}}\right) \\
= & : g_{\alpha, \mu}(s, l) .
\end{aligned}
$$


Since $g_{\alpha, \mu}(s, l)$ satisfies (H2), we can apply Lemma 2.15 to get

$$
\begin{aligned}
f\left(\mathbb{A}^{+}\right)^{3} & =\operatorname{Res}_{s=0} \sum_{\left(l_{1}, l_{2}\right) \in\left(\mathbb{Z}^{n}\right)^{2}} f(s, l) \\
& =\sum_{\left(l_{1}, l_{2}\right) \in\left(\mathbb{Z}^{n}\right)^{2}} \operatorname{Res}_{s=0} g_{\alpha, \mu}(s, l) \operatorname{Tr}\left(\gamma^{\alpha_{3}} \gamma^{\mu_{3}} \gamma^{\alpha_{2}} \gamma^{\mu_{2}} \gamma^{\alpha_{1}} \gamma^{\mu_{1}}\right)=: \sum_{l} X_{l} .
\end{aligned}
$$

Recall that $l_{3}:=-l_{1}-l_{2}=-\hat{l}_{2}$. By (2.11) and (2.13),

$$
\begin{gathered}
\operatorname{Res}_{s=0} g_{\alpha, \mu}(s, l) i e^{\frac{i}{2} l_{1} \Theta l_{2}} \tilde{a}_{\alpha, l}\left(2\left(-l_{1}^{i}+l_{3}^{i}\right) \frac{\pi^{2}}{12}\left(\delta_{\mu_{1} \mu_{2}} \delta_{\mu_{3} i}+\delta_{\mu_{1} \mu_{3}} \delta_{\mu_{2} i}+\delta_{\mu_{1} i} \delta_{\mu_{2} \mu_{3}}\right)\right. \\
\left.+\left(l_{1 \mu_{2}} \delta_{\mu_{1} \mu_{3}}-l_{3 \mu_{3}} \delta_{\mu_{1} \mu_{2}}\right) \frac{\pi^{2}}{2}\right)
\end{gathered}
$$

We decompose $X_{l}$ in five terms: $X_{l}=2^{m} \frac{\pi^{2}}{2} i e^{\frac{i}{2} l_{1} \Theta l_{2}} \tilde{a}_{\alpha, l}\left(T_{1}+T_{2}+T_{3}+T_{4}+T_{5}\right)$ where

$$
\begin{aligned}
& T_{0}:=\frac{1}{3}\left(-l_{1}^{i}+l_{3}^{i}\right)\left(\delta_{\mu \nu} \delta_{\rho i}+\delta_{\mu \rho} \delta_{v i}+\delta_{\mu i} \delta_{v \rho}\right)+l_{1 v} \delta_{\mu \rho}-l_{3 \rho} \delta_{\mu v}, \\
& T_{1}:=\left(\delta^{\alpha_{3} \rho} \delta^{\alpha_{2} v} \delta^{\alpha_{1} \mu}-\delta^{\alpha_{3} \rho} \delta^{\alpha_{2} \alpha_{1}} \delta^{\mu v}+\delta^{\alpha_{3} \rho} \delta^{\alpha_{2} \mu} \delta^{\alpha_{1} v}\right) T_{0}, \\
& T_{2}:=\left(-\delta^{\alpha_{2} \alpha_{3}} \delta^{\rho v} \delta^{\alpha_{1} \mu}+\delta^{\alpha_{2} \alpha_{3}} \delta^{\alpha_{1} \rho} \delta^{\mu v}-\delta^{\alpha_{2} \alpha_{3}} \delta^{\rho \mu} \delta^{\alpha_{1} v}\right) T_{0}, \\
& T_{3}:=\left(\delta^{\alpha_{3} v} \delta^{\alpha_{2} \rho} \delta^{\alpha_{1} \mu}-\delta^{\alpha_{3} v} \delta^{\alpha_{1} \rho} \delta^{\alpha_{2} \mu}+\delta^{\alpha_{3} v} \delta^{\rho \mu} \delta^{\alpha_{1} \alpha_{2}}\right) T_{0}, \\
& T_{4}:=\left(-\delta^{\alpha_{1} \alpha_{3}} \delta^{\alpha_{2} \rho} \delta^{\mu v}+\delta^{\alpha_{1} \alpha_{3}} \delta^{\rho v} \delta^{\alpha_{2} \mu}-\delta^{\alpha_{1} \alpha_{3}} \delta^{\rho \mu} \delta^{\alpha_{2} v}\right) T_{0}, \\
& T_{5}:=\left(\delta^{\alpha_{3} \mu} \delta^{\alpha_{2} \rho} \delta^{\alpha_{1} v}-\delta^{\alpha_{3} \mu} \delta^{\rho v} \delta^{\alpha_{1} \alpha_{2}}+\delta^{\alpha_{3} \mu} \delta^{\alpha_{1} \rho} \delta^{\alpha_{2} v}\right) T_{0} .
\end{aligned}
$$

Let $p:=-l_{1}-2 l_{3}, q:=2 l_{1}+l_{3}, r:=-p-q=-l_{1}+l_{3}$. We compute each $T_{i}$ and find that

$$
\begin{aligned}
3 T_{1}= & \delta^{\alpha_{1} \alpha_{2}}\left(2-2^{m}\right) p^{\alpha_{3}}+\delta^{\alpha_{3} \alpha_{1}} q^{\alpha_{2}}-\delta^{\alpha_{2} \alpha_{1}} q^{\alpha_{3}}+\delta^{\alpha_{3} \alpha_{2}} q^{\alpha_{1}}+\delta^{\alpha_{3} \alpha_{2}} r^{\alpha_{1}} \\
& -\delta^{\alpha_{2} \alpha_{1}} r^{\alpha_{3}}+\delta^{\alpha_{3} \alpha_{1}} r^{\alpha_{2}}, \\
3 T_{2}= & \left(2^{m}-2\right) \delta^{\alpha_{2} \alpha_{3}} p^{\alpha_{1}}-2^{m} \delta^{\alpha_{2} \alpha_{3}} q^{\alpha_{1}}-2^{m} \delta^{\alpha_{2} \alpha_{3}} r^{\alpha_{1}}, \\
3 T_{3}= & \delta^{\alpha_{1} \alpha_{3}} p^{\alpha_{2}}-\delta^{\alpha_{2} \alpha_{3}} p^{\alpha_{1}}+\delta^{\alpha_{1} \alpha_{2}} p^{\alpha_{3}}+2^{m} \delta^{\alpha_{2} \alpha_{1}} q^{\alpha_{3}}+\delta^{\alpha_{3} \alpha_{2}} r^{\alpha_{1}} \\
& -\delta^{\alpha_{3} \alpha_{1}} r^{\alpha_{2}}+\delta^{\alpha_{1} \alpha_{2}} r^{\alpha_{3}}, \\
3 T_{4}= & -\delta^{\alpha_{1} \alpha_{3}} 2^{m} p^{\alpha_{2}}-\delta^{\alpha_{1} \alpha_{3}} 2^{m} q^{\alpha_{2}}+\delta^{\alpha_{1} \alpha_{3}}\left(2^{m}-2\right) r^{\alpha_{2}}, \\
3 T_{5}= & \delta^{\alpha_{1} \alpha_{3}} p^{\alpha_{2}}-\delta^{\alpha_{1} \alpha_{2}} p^{\alpha_{3}}+\delta^{\alpha_{3} \alpha_{2}} p^{\alpha_{1}}+\delta^{\alpha_{3} \alpha_{2}} q^{\alpha_{1}}-\delta^{\alpha_{1} \alpha_{2}} q^{\alpha_{3}}+\delta^{\alpha_{3} \alpha_{1}} q^{\alpha_{2}} \\
& +\left(2-2^{m}\right) \delta^{\alpha_{1} \alpha_{2}} r^{\alpha_{3}} .
\end{aligned}
$$

Thus,

$$
X_{l}=2^{m} \frac{2 \pi^{2}}{3} i e^{\frac{i}{2} l_{1} \cdot \Theta l_{2}} \tilde{a}_{\alpha, l}\left(q^{\alpha_{3}} \delta^{\alpha_{1} \alpha_{2}}+r^{\alpha_{2}} \delta^{\alpha_{1} \alpha_{3}}+p^{\alpha_{1}} \delta^{\alpha_{2} \alpha_{3}}\right)
$$


and

$$
f\left(\mathbb{A}^{+}\right)^{3}=i 2 c\left(S_{1}+S_{2}+S_{3}\right)
$$

where $S_{1}, S_{2}$ and $S_{3}$ correspond to $q^{\alpha_{3}} \delta^{\alpha_{1} \alpha_{2}}, r^{\alpha_{2}} \delta^{\alpha_{1} \alpha_{3}}$ and $p^{\alpha_{1}} \delta^{\alpha_{2} \alpha_{3}}$, respectively. In $S_{1}$ we permute the $l_{i}$ variables the following way: $l_{1} \mapsto l_{3}, l_{2} \mapsto l_{1}, l_{3} \mapsto l_{2}$. Therefore, $l_{3} \cdot \Theta l_{1} \mapsto l_{3} \cdot \Theta l_{1}$ and $q \mapsto r$. With a similar permutation of the $\alpha_{i}$, we see that $S_{1}=S_{2}$. We apply the same principles to prove that $S_{1}=S_{3}$ (using permutation $\left.l_{1} \mapsto l_{2}, l_{2} \mapsto l_{3}, l_{3} \mapsto l_{1}\right)$. Thus,

$$
\frac{1}{3} f\left(\mathbb{A}^{+}\right)^{3}=i 2 c \sum_{l_{i}} \tilde{a}_{\alpha, l} e^{\frac{i}{2} l_{1} \cdot \Theta l_{2}}\left(l_{1}-l_{2}\right)^{\alpha_{3}} \delta^{\alpha_{1} \alpha_{2}}=S_{4}-S_{5},
$$

where $S_{4}$ correspond to $l_{1}$ and $S_{5}$ to $l_{2}$. We permute the $l_{i}$ variables in $S_{5}$ as follows: $l_{1} \mapsto l_{2}, l_{2} \mapsto l_{1}, l_{3} \mapsto l_{3}$, with a similar permutation on the $\alpha_{i}$. Since $l_{1} \cdot \Theta l_{2} \mapsto$ $-l_{1} \cdot \Theta l_{2}$, we finally get

$$
\frac{1}{3} f\left(\mathbb{A}^{+}\right)^{3}=-4 c \sum_{l_{i}} a_{\alpha_{1}, l_{1}} a_{\alpha_{2}, l_{2}} a_{\alpha_{3},-l_{1}-l_{2}} \sin \frac{l_{1} \cdot \Theta l_{2}}{2} l_{1}^{\alpha_{3}} \delta^{\alpha_{1} \alpha_{2}} .
$$

(iii) Lemma 6.11 gives that $f \mathbb{A}^{++++}=\operatorname{Res}_{s=0} \sum_{\left(l_{1}, l_{2}, l_{3}\right) \in\left(\mathbb{Z}^{n}\right)^{3}} f_{\mu, \alpha}(s, l) \operatorname{Tr} \gamma^{\mu, \alpha}$ where

$$
\begin{aligned}
& \theta:=l_{1} \cdot \Theta l_{2}+l_{1} \cdot \Theta l_{3}+l_{2} \cdot \Theta l_{3}, \\
& \operatorname{Tr} \gamma^{\mu, \alpha}:=\operatorname{Tr}\left(\gamma^{\alpha_{4}} \gamma^{\mu_{4}} \gamma^{\alpha_{3}} \gamma^{\mu_{3}} \gamma^{\alpha_{2}} \gamma^{\mu 2} \gamma^{\alpha_{1}} \gamma^{\mu_{1}}\right), \\
& f_{\mu, \alpha}(s, l):=\sum_{k \in \mathbb{Z}^{n}}^{\prime} e^{\frac{i}{2} \theta} \frac{k_{\mu_{1}}\left(k+l_{1}\right)_{\mu_{2}}\left(k+\hat{l}_{2}\right)_{\mu_{3}}\left(k+\hat{l}_{3}\right)_{\mu_{4}}}{|k|^{s+2}\left|k+l_{1}\right|^{2}\left|k+\hat{l}_{2}\right|^{2}\left|k+\hat{l}_{3}\right|^{2}}, \\
& \tilde{a}_{\alpha, l}:=a_{\alpha_{1}, l_{1}} a_{\alpha_{2}, l_{2}} a_{\alpha_{3}, l_{3}} a_{\alpha_{4},-l_{1}-l_{2}-l_{3}} .
\end{aligned}
$$

Using (2.10) and Corollary 2.14 successively, we find

$$
\begin{aligned}
f_{\mu, \alpha}(s, l) & \sim \sum_{k \in \mathbb{Z}^{n}}^{\prime} e^{\frac{i}{2} \theta} \frac{k_{\mu_{1}} k_{\mu_{2}} k_{\mu_{3}} k_{\mu_{4}}}{|k|^{s+2}\left|k+l_{1}\right|^{2}\left|k+l_{1}+l_{2}\right|^{2}\left|k+l_{1}+l_{2}+l_{3}\right|^{2}} \tilde{a}_{\alpha, l} \\
& \sim \sum_{k \in \mathbb{Z}^{n}}^{\prime} e^{\frac{i}{2} \theta \frac{k_{\mu_{1}} k_{\mu_{2}} k_{\mu_{3}} k_{\mu_{4}}}{|k|^{s+8}} \tilde{a}_{\alpha, l} .}
\end{aligned}
$$

Since the function $\sum_{k \in \mathbb{Z}^{n}}^{\prime} e^{\frac{i}{2} \theta} \frac{k_{\mu_{1}} k_{\mu_{2}} k_{\mu_{3}} k_{\mu_{4}}}{|k|^{s+8}} \tilde{a}_{\alpha, l}$ satisfies (H2), Lemma $2.15 \mathrm{im}$ plies that

$$
f\left(\mathbb{A}^{+}\right)^{4}=\sum_{\left(l_{1}, l_{2}, l_{3}\right) \in\left(\mathbb{Z}^{n}\right)^{3}} e^{\frac{i}{2} \theta} \tilde{a}_{\alpha, l} \operatorname{Res}_{s=0} \sum_{k \in \mathbb{Z}^{n}}^{\prime} \frac{k_{\mu_{1}} k_{\mu_{2}} k_{\mu_{3}} k_{\mu_{4}}}{|k|^{s+8}} \operatorname{Tr} \gamma^{\mu, \alpha}=: \sum_{l} X_{l} .
$$


Therefore, with (2.13), we get $X_{l}=\frac{\pi^{2}}{12} \tilde{a}_{\alpha, l} e^{\frac{i}{2} \theta}(A+B+C)$, where

$$
\begin{aligned}
& A:=\operatorname{Tr}\left(\gamma^{\alpha_{4}} \gamma^{\mu_{4}} \gamma^{\alpha_{3}} \gamma_{\mu_{4}} \gamma^{\alpha_{2}} \gamma^{\mu_{2}} \gamma^{\alpha_{1}} \gamma_{\mu_{2}}\right), \\
& B:=\operatorname{Tr}\left(\gamma^{\alpha_{4}} \gamma^{\mu_{4}} \gamma^{\alpha_{3}} \gamma^{\mu_{2}} \gamma^{\alpha_{2}} \gamma_{\mu_{4}} \gamma^{\alpha_{1}} \gamma_{\mu_{2}}\right), \\
& C:=\operatorname{Tr}\left(\gamma^{\alpha_{4}} \gamma^{\mu_{4}} \gamma^{\alpha_{3}} \gamma_{\mu_{2}} \gamma^{\alpha_{2}} \gamma^{\mu_{2}} \gamma^{\alpha_{1}} \gamma_{\mu_{4}}\right) .
\end{aligned}
$$

Using successively $\left\{\gamma^{\mu}, \gamma^{\nu}\right\}=2 \delta^{\mu \nu}$ and $\gamma^{\mu} \gamma_{\mu}=2^{m} 1_{2^{m}}$, we find that

$$
\begin{aligned}
& A=C=4 \operatorname{Tr}\left(\gamma^{\alpha_{4}} \gamma^{\alpha_{3}} \gamma^{\alpha_{2}} \gamma^{\alpha_{1}}\right), \\
& B=-4\left(\operatorname{Tr}\left(\gamma^{\alpha_{4}} \gamma^{\alpha_{3}} \gamma^{\alpha_{1}} \gamma^{\alpha_{2}}\right)+\operatorname{Tr}\left(\gamma^{\alpha_{4}} \gamma^{\alpha_{2}} \gamma^{\alpha_{3}} \gamma^{\alpha_{1}}\right)\right) .
\end{aligned}
$$

Thus, $A+B+C=8 \cdot 2^{m}\left(\delta^{\alpha_{4} \alpha_{3}} \delta^{\alpha_{2} \alpha_{1}}+\delta^{\alpha_{4} \alpha_{1}} \delta^{\alpha_{3} \alpha_{2}}-2 \delta^{\alpha_{4} \alpha_{2}} \delta^{\alpha_{3} \alpha_{1}}\right)$ and

$$
X_{l}=\frac{2 \pi^{2}}{3} 2^{m} e^{\frac{i}{2} \theta} \tilde{a}_{\alpha, l}\left(\delta^{\alpha_{4} \alpha_{3}} \delta^{\alpha_{2} \alpha_{1}}+\delta^{\alpha_{4} \alpha_{1}} \delta^{\alpha_{3} \alpha_{2}}-2 \delta^{\alpha_{4} \alpha_{2}} \delta^{\alpha_{3} \alpha_{1}}\right) .
$$

By (6.7) we get

$$
f\left(\mathbb{A}^{+}\right)^{4}=2 c\left(-2 T_{1}+T_{2}+T_{3}\right)
$$

where

$$
\begin{aligned}
T_{1} & :=\sum_{l_{1}, \ldots, l_{4}} a_{\alpha_{4}, l_{4}} a_{\alpha_{3}, l_{3}} a_{\alpha_{2}, l_{2}} a_{\alpha_{1}, l_{1}} e^{\frac{i}{2} \theta} \delta_{0, \sum_{i} l_{i}} \delta^{\alpha_{4} \alpha_{2}} \delta^{\alpha_{3} \alpha_{1}}, \\
T_{2} & :=\sum_{l_{1}, \ldots, l_{4}} a_{\alpha_{4}, l_{4}} a_{\alpha_{3}, l_{3}} a_{\alpha_{2}, l_{2}} a_{\alpha_{1}, l_{1}} e^{\frac{i}{2} \theta} \delta_{0, \sum_{i} l_{i}} \delta^{\alpha_{4} \alpha_{3}} \delta^{\alpha_{2} \alpha_{1}}, \\
T_{3} & :=\sum_{l_{1}, \ldots, l_{4}} a_{\alpha_{4}, l_{4}} a_{\alpha_{3}, l_{3}} a_{\alpha_{2}, l_{2}} a_{\alpha_{1}, l_{1}} e^{\frac{i}{2} \theta} \delta_{0, \sum_{i} l_{i}} \delta^{\alpha_{4} \alpha_{1}} \delta^{\alpha_{3} \alpha_{2}} .
\end{aligned}
$$

We now proceed to the following permutations of the $l_{i}$ variables in the $T_{1}$ term: $l_{1} \mapsto l_{2}, l_{2} \mapsto l_{1}, l_{3} \mapsto l_{4}, l_{4} \mapsto l_{3}$. While $\sum_{i} l_{i}$ is invariant, $\theta$ is modified: $\theta \mapsto l_{2} \cdot \Theta l_{1}+l_{2} \cdot \Theta l_{4}+l_{1} \cdot \Theta l_{4}$. With $\delta_{0, \sum_{i} l_{i}}$ in factor, we can let $l_{4}$ be $-l_{1}-l_{2}-l_{3}$ so that $\theta \mapsto-\theta$. We also permute the $\alpha_{i}$ in the same way. Thus,

$$
T_{1}=\sum_{l_{1}, \ldots, l_{4}} a_{\alpha_{3}, l_{3}} a_{\alpha_{4}, l_{4}} a_{\alpha_{1}, l_{1}} a_{\alpha_{2}, l_{2}} e^{-\frac{i}{2} \theta} \delta_{0, \sum_{i} l_{i}} \delta^{\alpha_{3} \alpha_{1}} \delta^{\alpha_{4} \alpha_{2}} .
$$

Therefore,

$$
2 T_{1}=2 \sum_{l_{1}, \ldots, l_{4}} a_{\alpha_{4}, l_{4}} a_{\alpha_{3}, l_{3}} a_{\alpha_{2}, l_{2}} a_{\alpha_{1}, l_{1}} \cos \frac{\theta}{2} \delta_{0, \sum_{i} l_{i}} \delta^{\alpha_{4} \alpha_{2}} \delta^{\alpha_{3} \alpha_{1}} .
$$

The same principles are applied to $T_{2}$ and $T_{3}$. Namely, the permutation $l_{1} \mapsto l_{1}$, $l_{2} \mapsto l_{3}, l_{3} \mapsto l_{2}, l_{4} \mapsto l_{4}$ in $T_{2}$ and the permutation $l_{1} \mapsto l_{2}, l_{2} \mapsto l_{3}, l_{3} \mapsto l_{1}$, 
$l_{4} \mapsto l_{4}$ in $T_{3}$ (the $\alpha_{i}$ variables are permuted the same way) give

$$
\begin{aligned}
T_{2} & =\sum_{l_{1}, \ldots, l_{4}} a_{\alpha_{4}, l_{4}} a_{\alpha_{3}, l_{3}} a_{\alpha_{2}, l_{2}} a_{\alpha_{1}, l_{1}} e^{\frac{i}{2} \phi} \delta_{0, \sum_{i} l_{i}} \delta^{\alpha_{4} \alpha_{2}} \delta^{\alpha_{3} \alpha_{1}}, \\
T_{3} & =\sum_{l_{1}, \ldots, l_{4}} a_{\alpha_{4}, l_{4}} a_{\alpha_{3}, l_{3}} a_{\alpha_{2}, l_{2}} a_{\alpha_{1}, l_{1}} e^{-\frac{i}{2} \phi} \delta_{0, \sum_{i} l_{i}} \delta^{\alpha_{4} \alpha_{2}} \delta^{\alpha_{3} \alpha_{1}},
\end{aligned}
$$

where $\phi:=l_{1} \cdot \Theta l_{2}+l_{1} \cdot \Theta l_{3}-l_{2} \cdot \Theta l_{3}$. Finally we get

$$
\begin{aligned}
f\left(\mathbb{A}^{+}\right)^{4} & =4 c \sum_{l_{1}, \ldots, l_{4}} a_{\alpha_{1}, l_{4}} a_{\alpha_{2}, l_{3}} a_{l_{2}}^{\alpha_{1}} a_{l_{1}}^{\alpha_{2}} \delta_{0, \sum_{i} l_{i}}\left(\cos \frac{\phi}{2}-\cos \frac{\theta}{2}\right) \\
& =8 c \sum_{l_{1}, \ldots, l_{3}} a_{\alpha_{1},-l_{1}-l_{2}-l_{3}} a_{\alpha_{2}, l_{3}} a_{l_{2}}^{\alpha_{1}} a_{l_{1}}^{\alpha_{2}} \sin \frac{l_{1} \cdot \Theta\left(l_{2}+l_{3}\right)}{2} \sin \frac{l_{2} \cdot \Theta l_{3}}{2} .
\end{aligned}
$$

(iv) Suppose that $q=2$. By Lemma 6.11 we get

$$
f \mathbb{A}^{\sigma}=\operatorname{Res}_{s=0} \sum_{l \in \mathbb{Z}^{n}} \lambda_{\sigma} f_{\alpha, \mu}(s, l) \operatorname{Tr}\left(\gamma^{\alpha_{2}} \gamma^{\mu_{2}} \gamma^{\alpha_{1}} \gamma^{\mu_{1}}\right)
$$

where

$$
f_{\alpha, \mu}(s, l):=\sum_{k \in \mathbb{Z}^{n}}^{\prime} \frac{k_{\mu_{1}}(k+l)_{\mu_{2}}}{|k|^{s+2}|k+l|^{2}} e^{i \eta k \cdot \Theta l} \tilde{a}_{\alpha, l}
$$

and $\eta:=\frac{1}{2}\left(\sigma_{1}-\sigma_{2}\right) \in\{-1,1\}$. As in the proof of (i), since the presence of the phase does not change the fact that $r(s, l)$ satisfies $(\mathrm{H} 1)$, we get

$$
f_{\alpha, \mu}(s, l) \sim f_{1}(s, l)-f_{2}(s, l)+f_{3}(s, l)
$$

where

$$
\begin{aligned}
& f_{1}(s, l)=\sum_{k \in \mathbb{Z}^{n}}^{\prime} \frac{k_{\mu_{1}}(k+l)_{\mu_{2}}}{|k|^{s+4}} e^{i \eta k \cdot \Theta l} \tilde{a}_{\alpha, l}, \\
& f_{2}(s, l)=\sum_{k \in \mathbb{Z}^{n}}^{\prime} \frac{k_{\mu_{1}}(k+l)_{\mu_{2}}\left(2 k \cdot l+|l|^{2}\right)}{|k|^{s+6}} e^{i \eta k \cdot \Theta l} \tilde{a}_{\alpha, l}, \\
& f_{3}(s, l)=\sum_{k \in \mathbb{Z}^{n}} \frac{k_{\mu_{1}}(k+l)_{\mu_{2}}\left(2 k \cdot l+|l|^{2}\right)^{2}}{|k|^{s+8}} e^{i \eta k \cdot \Theta l} \tilde{a}_{\alpha, l} .
\end{aligned}
$$

Suppose that $l=0$. Then $f_{2}(s, 0)=f_{3}(s, 0)=0$ and Proposition 2.1 implies that

$$
f_{1}(s, 0)=\sum_{k \in \mathbb{Z}^{n}}^{\prime} \frac{k_{\mu_{1}} k_{\mu_{2}}}{|k|^{s+4}} \tilde{a}_{\alpha, 0}
$$


is holomorphic at 0 and so is $f_{\alpha, \mu}(s, 0)$.

Since $\frac{1}{2 \pi} \Theta$ is diophantine, Theorem 2.63 gives us the result.

Suppose that $q=3$. Then Lemma 6.11 implies that

$$
f \mathbb{A}^{\sigma}=\operatorname{Res}_{s=0} \sum_{l \in\left(\mathbb{Z}^{n}\right)^{2}} f_{\mu, \alpha}(s, l) \operatorname{Tr}\left(\gamma^{\mu_{3}} \gamma^{\alpha_{3}} \ldots \gamma^{\mu_{1}} \gamma^{\alpha_{1}}\right)
$$

where

$$
f_{\mu, \alpha}(s, l):=\sum_{k \in \mathbb{Z}^{n}}^{\prime} \lambda_{\sigma} e^{i k \cdot \Theta\left(\varepsilon_{1} l_{1}+\varepsilon_{2} l_{2}\right)} e^{\frac{i}{2} \sigma_{2} l_{1} \cdot \Theta l_{2}} \frac{k_{\mu_{1}}\left(k+l_{1}\right)_{\mu_{2}}\left(k+l_{1}+l_{2}\right) \mu_{3}}{|k|^{s+2}\left|k+l_{1}\right|^{2}\left|k+l_{1}+l_{2}\right|^{2}} \tilde{a}_{\alpha, l}
$$

and $\varepsilon_{i}:=\frac{1}{2}\left(\sigma_{i}-\sigma_{3}\right) \in\{-1,0,1\}$. By hypothesis $\left(\varepsilon_{1}, \varepsilon_{2}\right) \neq(0,0)$. There are six possibilities for the values of $\left(\varepsilon_{1}, \varepsilon_{2}\right)$ corresponding to the six possibilities for the values of $\sigma:(-,-,+),(-,+,+),(+,-,+),(+,+,-),(-,+,-)$, and $(+,-,-)$. As in (ii), we see that

$$
\begin{aligned}
& f_{\mu, \alpha}(s, l) \\
& \sim\left(\sum_{k \in \mathbb{Z}^{n}}^{\prime} \frac{e^{i k \cdot \Theta\left(\varepsilon_{1} l_{1}+\varepsilon_{2} l_{2}\right)} k_{\mu_{1}}\left(k+l_{1}\right)_{\mu_{2}}\left(k+\hat{l}_{2}\right)_{\mu_{3}}}{|k|^{s+6}}\right. \\
& \left.-\sum_{k \in \mathbb{Z}^{n}}^{\prime} \frac{e^{i k \cdot \Theta\left(\varepsilon_{1} l_{1}+\varepsilon_{2} l_{2}\right)} k_{\mu_{1}}\left(k+l_{1}\right)_{\mu_{2}}\left(k+\hat{l}_{2}\right)_{\mu_{3}}\left(2 k \cdot l_{1}+2 k \cdot \hat{l}_{2}\right)}{|k|^{s+8}} \lambda_{\sigma} \tilde{a}_{\alpha, l} e^{\frac{i}{2} \sigma_{2} l_{1} \cdot \Theta l_{2}}\right) .
\end{aligned}
$$

With $Z:=\left\{\left(l_{1}, l_{2}\right) \mid \varepsilon_{1} l_{1}+\varepsilon_{2} l_{2}=0\right\}$, it follows from Theorem 2.6 (iii) that $\sum_{l \in\left(\mathbb{Z}^{n}\right)^{2} \backslash Z} f_{\mu, \alpha}(s, l)$ is holomorphic at 0 . To conclude we need to prove that

$$
\sum_{\sigma} g(\sigma):=\sum_{\sigma} \sum_{l \in Z} f_{\mu, \alpha}(s, l) \operatorname{Tr}\left(\gamma^{\mu_{3}} \gamma^{\alpha_{3}} \ldots \gamma^{\mu_{1}} \gamma^{\alpha_{1}}\right)
$$

is holomorphic at 0 . By definition, $\lambda_{\sigma}=i \sigma_{1} \sigma_{2} \sigma_{3}$ and as a consequence, we check that

$$
\begin{aligned}
& g(-,-,+)=-g(+,+,-), \\
& g(+,-,+)=-g(+,-,-), \\
& g(-,+,+)=-g(-,+,-),
\end{aligned}
$$

which implies that $\sum_{\sigma} g(\sigma)=0$. The result follows.

Suppose finally that $q=4$. Again Lemma 6.11 implies that

$$
f \mathbb{A}^{\sigma}=\operatorname{Res}_{s=0} \sum_{l \in\left(\mathbb{Z}^{n}\right)^{3}} f_{\mu, \alpha}(s, l) \operatorname{Tr}\left(\gamma^{\mu_{4}} \gamma^{\alpha_{4}} \ldots \gamma^{\mu_{1}} \gamma^{\alpha_{1}}\right),
$$


where

$$
\begin{aligned}
f_{\mu, \alpha}(s, l):= & \sum_{k \in \mathbb{Z}^{n}}^{\prime} \lambda_{\sigma} e^{i k \cdot \Theta \sum_{i=1}^{3} \varepsilon_{i} l_{i}} e^{\frac{i}{2}\left(\sigma_{2} l_{1} \cdot \Theta l_{2}+\sigma_{3}\left(l_{1}+l_{2}\right) \cdot \Theta l_{3}\right)} \\
& \frac{k_{\mu_{1}}\left(k+l_{1}\right)_{\mu_{2}}\left(k+l_{1}+l_{2}\right)_{\mu_{3}}\left(k+l_{1}+l_{2}+l_{3}\right)_{\mu_{4}}}{|k|^{s+2}\left|k+l_{1}\right|^{2}\left|k+l_{1}+l_{2}\right|^{2}\left|k+l_{1}+l_{2}+l_{3}\right|^{2}} \tilde{a}_{\alpha, l}
\end{aligned}
$$

and $\varepsilon_{i}:=\frac{1}{2}\left(\sigma_{i}-\sigma_{4}\right) \in\{-1,0,1\}$. By hypothesis, $\left(\varepsilon_{1}, \varepsilon_{2}, \varepsilon_{3}\right) \neq(0,0,0)$. There are fourteen possibilities for the values of $\left(\varepsilon_{1}, \varepsilon_{2}, \varepsilon_{3}\right)$ corresponding to the fourteen possibilities for the values of $\sigma:(-,-,-,+),(-,-,+,+),(-,+,-,+),(+,-,-,+)$, $(-,+,+,+),(+,-,+,+),(+,+,-,+),(+,+,+,-),(-,-,+,-),(-,+,-,-)$, $(+,-,-,-),(-,+,+,-),(+,-,+,-)$ and $(+,+,-,-)$. As in (ii), we see that, with $\theta_{\sigma}:=\sigma_{2} l_{1} \cdot \Theta l_{2}+\sigma_{3}\left(l_{1}+l_{2}\right) \cdot \Theta l_{3}$,

$$
f_{\mu, \alpha}(s, l) \sim \sum_{k \in \mathbb{Z}^{n}}^{\prime} \lambda_{\sigma} e^{i k \cdot \Theta \sum_{i=1}^{3} \varepsilon_{i} l_{i}} e^{\frac{i}{2} \theta_{\sigma}} \frac{k_{\mu_{1}} k_{\mu_{2}} k_{\mu_{3}} k_{\mu_{4}}}{|k|^{s+8}} \tilde{a}_{\alpha, l}=: g_{\mu, \alpha}(s, l) \text {. }
$$

With $Z_{\sigma}:=\left\{\left(l_{1}, l_{2}, l_{3}\right) \mid \sum_{i=1}^{3} \varepsilon_{i} l_{i}=0\right\}$, it follows from Theorem 2.6 (iii) that the series $\sum_{l \in\left(\mathbb{Z}^{n}\right)^{3} \backslash Z_{\sigma}} f_{\mu, \alpha}(s, l)$ is holomorphic at 0 . To conclude, we need to prove that

$$
\sum_{\sigma} g(\sigma):=\sum_{\sigma} \operatorname{Res}_{s=0} \sum_{l \in Z_{\sigma}} g_{\mu, \alpha}(s, l) \operatorname{Tr}\left(\gamma^{\mu_{4}} \gamma^{\alpha_{4}} \ldots \gamma^{\mu_{1}} \gamma^{\alpha_{1}}\right)=0 .
$$

Let $C$ be the set of the fourteen values of $\sigma$ and $C_{7}$ be the set of the seven first values of $\sigma$ given above. Lemma 6.7 implies

$$
\sum_{\sigma \in C} g(\sigma)=2 \sum_{\sigma \in C_{7}} g(\sigma)
$$

Thus, in the following, we restrict to these seven values. Denote $\sum_{k \in \mathbb{Z}^{n}}^{\prime} \frac{k_{\mu_{1}} k_{\mu_{2}} k_{\mu_{3}} k_{\mu_{4}}}{|k|^{s+8}}$ by $F_{\mu}(s)$ so that

$$
g(\sigma)=\operatorname{Res}_{s=0} F_{\mu}(s) \lambda_{\sigma} \sum_{l \in Z_{\sigma}} e^{\frac{i}{2} \theta_{\sigma}} \tilde{a}_{\alpha, l} \operatorname{Tr}\left(\gamma^{\mu_{4}} \gamma^{\alpha_{4}} \ldots \gamma^{\mu_{1}} \gamma^{\alpha_{1}}\right) .
$$

Recall from (6.7) that

$$
\operatorname{Res}_{s=0} F_{\mu}(s) \operatorname{Tr}\left(\gamma^{\mu_{4}} \gamma^{\alpha_{4}} \ldots \gamma^{\mu_{1}} \gamma^{\alpha_{1}}\right)=2 c\left(\delta^{\alpha_{4} \alpha_{3}} \delta^{\alpha_{2} \alpha_{1}}+\delta^{\alpha_{4} \alpha_{1}} \delta^{\alpha_{3} \alpha_{2}}-2 \delta^{\alpha_{4} \alpha_{2}} \delta^{\alpha_{3} \alpha_{1}}\right) \text {. }
$$

As a consequence, we get, with $\tilde{a}_{\alpha, l}:=a_{\alpha_{1}, l_{1}} \ldots a_{\alpha_{4}, l_{4}}$,

$$
\begin{aligned}
g(\sigma)= & 2 c \lambda_{\sigma} \sum_{l \in\left(\mathbb{Z}^{n}\right)^{4}} e^{\frac{i}{2} \theta_{\sigma}} \tilde{a}_{\alpha, l} \delta_{\sum_{i=1}^{4} l_{i}, 0} \delta_{\sum_{i=1}^{3} \varepsilon_{i} l_{i}, 0} \\
& \cdot\left(\delta^{\alpha_{4} \alpha_{3}} \delta^{\alpha_{2} \alpha_{1}}+\delta^{\alpha_{4} \alpha_{1}} \delta^{\alpha_{3} \alpha_{2}}-2 \delta^{\alpha_{4} \alpha_{2}} \delta^{\alpha_{3} \alpha_{1}}\right) \\
= & : 2 c \lambda_{\sigma}\left(T_{1}+T_{2}-2 T_{3}\right) .
\end{aligned}
$$


We proceed to the following change of variables in $T_{1}: l_{1} \mapsto l_{1}, l_{2} \mapsto l_{3}, l_{3} \mapsto l_{2}$, $l_{4} \mapsto l_{4}$. Thus, we get $\theta_{\sigma} \mapsto \psi_{\sigma}:=\sigma_{2} l_{1} \cdot \Theta l_{3}+\sigma_{3}\left(l_{1}+l_{3}\right) \cdot \Theta l_{2}$ and $\sum_{i=1}^{3} \varepsilon_{i} l_{i} \mapsto$ $\varepsilon_{1} l_{1}+\varepsilon_{3} l_{2}+\varepsilon_{2} l_{3}=: u_{\sigma}(l)$. With a similar permutation of the $\alpha_{i}$, we obtain that

$$
T_{1}=\sum_{l \in\left(\mathbb{Z}^{n}\right)^{4}} e^{\frac{i}{2} \psi_{\sigma}} \tilde{a}_{\alpha, l} \delta_{\sum_{i=1}^{4} l_{i}, 0} \delta_{\varepsilon_{1} l_{1}+\varepsilon_{3} l_{2}+\varepsilon_{2} l_{3}, 0} \delta^{\alpha_{4} \alpha_{2}} \delta^{\alpha_{3} \alpha_{1}} .
$$

Next we proceed to the following change of variables in $T_{2}: l_{1} \mapsto l_{2}, l_{2} \mapsto l_{3}$, $l_{3} \mapsto l_{1}, l_{4} \mapsto l_{4}$. Thus, we get $\theta_{\sigma} \mapsto \phi_{\sigma}:=\sigma_{2} l_{2} \cdot \Theta l_{3}+\sigma_{3}\left(l_{2}+l_{3}\right) \cdot \Theta l_{1}$ and $\sum_{i=1}^{3} \varepsilon_{i} l_{i} \mapsto \varepsilon_{3} l_{1}+\varepsilon_{1} l_{2}+\varepsilon_{2} l_{3}=: v_{\sigma}(l)$. After a similar permutation of the $\alpha_{i}$, we have

$$
T_{2}=\sum_{l \in\left(\mathbb{Z}^{n}\right)^{4}} e^{\frac{i}{2} \phi_{\sigma}} \tilde{a}_{\alpha, l} \delta_{\sum_{i=1}^{4} l_{i}, 0} \delta_{\varepsilon_{3} l_{1}+\varepsilon_{1} l_{2}+\varepsilon_{2} l_{3}, 0} \delta^{\alpha_{4} \alpha_{2}} \delta^{\alpha_{3} \alpha_{1}} .
$$

Finally, we proceed to the following change of variables in $T_{3}: l_{1} \mapsto l_{2}, l_{2} \mapsto l_{1}$, $l_{3} \mapsto l_{4}, l_{4} \mapsto l_{3}$. Therefore we obtain $\theta_{\sigma} \mapsto-\theta_{\sigma}$ and $\sum_{i=1}^{3} \varepsilon_{i} l_{i} \mapsto\left(\varepsilon_{2}-\varepsilon_{3}\right) l_{1}+$ $\left(\varepsilon_{1}-\varepsilon_{3}\right) l_{2}-\varepsilon_{3} l_{3}=: w_{\sigma}(l)$. With a similar permutation of the $\alpha_{i}$, we have

$$
T_{3}=\sum_{l \in\left(\mathbb{Z}^{n}\right)^{4}} e^{-\frac{i}{2} \theta_{\sigma}} \tilde{a}_{\alpha, l} \delta_{\sum_{i=1}^{4} l_{i}, 0} \delta_{\left(\varepsilon_{2}-\varepsilon_{3}\right) l_{1}+\left(\varepsilon_{1}-\varepsilon_{3}\right) l_{2}-\varepsilon_{3} l_{3}, 0} \delta^{\alpha_{4} \alpha_{2}} \delta^{\alpha_{3} \alpha_{1}} .
$$

As a consequence, we get

$$
g(\sigma)=2 c \sum_{l \in\left(\mathbb{Z}^{n}\right)^{4}} K_{\sigma}\left(l_{1}, l_{2}, l_{3}\right) \tilde{a}_{\alpha, l} \delta_{\sum_{i=1}^{4} l_{i}, 0} \delta^{\alpha_{4} \alpha_{2}} \delta^{\alpha_{3} \alpha_{1}},
$$

where

$$
\begin{aligned}
& K_{\sigma}\left(l_{1}, l_{2}, l_{3}\right) \\
& \quad=\lambda_{\sigma}\left(e^{\frac{i}{2} \psi_{\sigma}} \delta_{u_{\sigma}(l), 0}+e^{\frac{i}{2} \phi_{\sigma}} \delta_{v_{\sigma}(l), 0}-e^{\frac{i}{2} \theta_{\sigma}} \delta_{\sum_{i=1}^{3} \varepsilon_{i} l_{i}, 0}-e^{-\frac{i}{2} \theta_{\sigma}} \delta_{w_{\sigma}(l), 0}\right) .
\end{aligned}
$$

The computation of $K_{\sigma}\left(l_{1}, l_{2}, l_{3}\right)$ for the seven values of $\sigma$ yields

$$
\begin{gathered}
K_{--++}\left(l_{1}, l_{2}, l_{3}\right)=\delta_{l_{1}+l_{3}, 0}+\delta_{l_{2}+l_{3}, 0}-\delta_{l_{1}+l_{2}, 0}-\delta_{l_{1}+l_{2}, 0}, \\
K_{-+-+}\left(l_{1}, l_{2}, l_{3}\right)=\delta_{l_{1}+l_{2}, 0}+\delta_{l_{1}+l_{2}, 0}-\delta_{l_{1}+l_{3}, 0}-\delta_{l_{1}+l_{3}, 0}, \\
K_{--++}\left(l_{1}, l_{2}, l_{3}\right)=\delta_{l_{2}+l_{3}, 0}+\delta_{l_{1}+l_{3}, 0}-\delta_{l_{2}+l_{3}, 0}-\delta_{l_{2}+l_{3}, 0}, \\
K_{---+}\left(l_{1}, l_{2}, l_{3}\right)=-\left(e^{\frac{i}{2} l_{1} \cdot \Theta l_{2}} \delta_{\sum_{i=1}^{3} l_{i}, 0}+e^{\frac{i}{2} l_{2} \cdot \Theta l_{1}} \delta_{\sum_{i=1}^{3} l_{i}, 0}\right. \\
\left.-e^{\frac{i}{2} l_{2} \cdot \Theta l_{1}} \delta_{\sum_{i=1}^{3} l_{i}, 0}-e^{\frac{i}{2} l_{1} \cdot \Theta l_{2}} \delta_{l_{3}, 0}\right), \\
K_{-+++}\left(l_{1}, l_{2}, l_{3}\right)=-\left(e^{\frac{i}{2} l_{3} \cdot \Theta l_{2}} \delta_{l_{1}, 0}+e^{\frac{i}{2} l_{3} \cdot \Theta l_{1}} \delta_{l_{2}, 0}\right. \\
\left.-e^{\frac{i}{2} l_{2} \cdot \Theta l_{3}} \delta_{l_{1}, 0}-e^{\frac{i}{2} l_{3} \cdot \Theta l_{1}} \delta_{l_{2}, 0}\right),
\end{gathered}
$$




$$
\begin{aligned}
K_{+-++}\left(l_{1}, l_{2}, l_{3}\right)=- & \left(e^{\frac{i}{2} l_{1} \cdot \Theta l_{2}} \delta_{l_{3}, 0}+e^{\frac{i}{2} l_{2} \cdot \Theta l_{1}} \delta_{l_{3}, 0}\right. \\
& \left.-e^{\frac{i}{2} l_{1} \cdot \Theta l_{3}} \delta_{l_{2}, 0}-e^{\frac{i}{2} l_{3} \cdot \Theta l_{2}} \delta_{l_{1}, 0}\right), \\
K_{++-+}\left(l_{1}, l_{2}, l_{3}\right)=- & \left(e^{\frac{i}{2} l_{1} \cdot \Theta l_{3}} \delta_{l_{2}, 0}+e^{\frac{i}{2} l_{2} \cdot \Theta l_{3}} \delta_{l_{1}, 0}\right. \\
& \left.-e^{\frac{i}{2} l_{1} \cdot \Theta l_{2}} \delta_{l_{3}, 0}-e^{\frac{i}{2} l_{2} \cdot \Theta l_{1}} \delta_{\sum_{i=1}^{3} l_{i}, 0}\right) .
\end{aligned}
$$

Thus,

$$
\sum_{\sigma \in C_{7}} K_{\sigma}\left(l_{1}, l_{2}, l_{3}\right)=2 i\left(\delta_{l_{3}, 0}-\delta_{\sum_{i=1}^{3} l_{i}, 0}\right) \sin \frac{l_{1} \cdot \Theta l_{2}}{2}
$$

and

$$
\sum_{\sigma \in C_{7}} g(\sigma)=i 4 c \sum_{l \in\left(\mathbb{Z}^{n}\right)^{4}}\left(\delta_{l_{3}, 0}-\delta_{\sum_{i=1}^{3} l_{i}, 0}\right) \sin \frac{l_{1} \cdot \Theta l_{2}}{2} \tilde{a}_{\alpha, l} \delta_{\sum_{i=1}^{4} l_{i}, 0} \delta^{\alpha_{4} \alpha_{2}} \delta^{\alpha_{3} \alpha_{1}} .
$$

The following change of variables $l_{1} \mapsto l_{2}, l_{1} \mapsto l_{2}, l_{3} \mapsto l_{4}, l_{4} \mapsto l_{3}$ gives

$$
\begin{aligned}
\sum_{l \in\left(\mathbb{Z}^{n}\right)^{4}} \delta_{\sum_{1}^{3} l_{i}, 0} \sin \frac{l_{1} \cdot \Theta l_{2}}{2} \tilde{a}_{\alpha, l} \delta_{\sum_{1}^{4} l_{i}, 0} \delta^{\alpha_{4} \alpha_{2}} \delta^{\alpha_{3} \alpha_{1}} \\
=-\sum_{l \in\left(\mathbb{Z}^{n}\right)^{4}} \delta_{l_{3}, 0} \sin \frac{l_{1} \cdot \Theta l_{2}}{2} \tilde{a}_{\alpha, l} \delta_{\sum_{1}^{4} l_{i}, 0} \delta^{\alpha_{4} \alpha_{2}} \delta^{\alpha_{3} \alpha_{1}},
\end{aligned}
$$

and hence

$$
\sum_{\sigma \in C_{7}} g(\sigma)=i 8 c \sum_{l \in\left(\mathbb{Z}^{n}\right)^{4}} \delta_{l_{3}, 0} \sin \frac{l_{1} \cdot \Theta l_{2}}{2} \tilde{a}_{\alpha, l} \delta_{\sum_{1}^{4} l_{i}, 0} \delta^{\alpha_{4} \alpha_{2}} \delta^{\alpha_{3} \alpha_{1}} .
$$

Finally, the change of variables $l_{2} \mapsto l_{4}, l_{4} \mapsto l_{2}$ gives

$$
\begin{aligned}
\sum_{l \in\left(\mathbb{Z}^{n}\right)^{4}} \delta_{l_{3}, 0} \sin \frac{l_{1} \cdot \Theta l_{2}}{2} \tilde{a}_{\alpha, l} \delta_{\sum_{1}^{4} l_{i}, 0} \delta^{\alpha_{4} \alpha_{2}} \delta^{\alpha_{3} \alpha_{1}} \\
=-\sum_{l \in\left(\mathbb{Z}^{n}\right)^{4}} \delta_{l_{3}, 0} \sin \frac{l_{1} \cdot \Theta l_{2}}{2} \tilde{a}_{\alpha, l} \delta_{\sum_{1}^{4} l_{i}, 0} \delta^{\alpha_{4} \alpha_{2}} \delta^{\alpha_{3} \alpha_{1}},
\end{aligned}
$$

which implies that $\sum_{\sigma \in C_{7}} g(\sigma)=0$.

Lemma 6.13. Suppose that $n=4$ and $\frac{1}{2 \pi} \Theta$ is diophantine. For any selfadjoint 1-form $A$ we have

$$
\zeta_{D_{A}}(0)-\zeta_{D}(0)=-c \tau\left(F_{\alpha_{1}, \alpha_{2}} F^{\alpha_{1} \alpha_{2}}\right) .
$$

Proof. By (4.5) and Lemma 6.6 we get

$$
\zeta_{D_{A}}(0)-\zeta_{D}(0)=\sum_{q=1}^{n} \frac{(-1)^{q}}{q} \sum_{\sigma \in\{+,-\}^{q}} f \mathbb{A}^{\sigma} .
$$


By Lemma 6.12 (iv), we see that the crossed terms all vanish. Thus, with Lemma 6.7, we get

$$
\zeta_{D_{A}}(0)-\zeta_{D}(0)=2 \sum_{q=1}^{n} \frac{(-1)^{q}}{q} f\left(\mathbb{A}^{+}\right)^{q}
$$

By definition,

$$
\begin{aligned}
F_{\alpha_{1} \alpha_{2}} & =i \sum_{k}\left(a_{\alpha_{2}, k} k_{\alpha_{1}}-a_{\alpha_{1}, k} k_{\alpha_{2}}\right) U_{k}+\sum_{k, l} a_{\alpha_{1}, k} a_{\alpha_{2}, l}\left[U_{k}, U_{l}\right] \\
& =i \sum_{k}\left[\left(a_{\alpha_{2}, k} k_{\alpha_{1}}-a_{\alpha_{1}, k} k_{\alpha_{2}}\right)-2 \sum_{l} a_{\alpha_{1}, k-l} a_{\alpha_{2}, l} \sin \left(\frac{k \cdot \Theta l}{2}\right)\right] U_{k} .
\end{aligned}
$$

Thus

$$
\begin{aligned}
\tau\left(F_{\alpha_{1} \alpha_{2}} F^{\alpha_{1} \alpha_{2}}\right) & \\
= & \sum_{\alpha_{1}, \alpha_{2}=1}^{2^{m}} \sum_{k \in \mathbb{Z}^{4}}\left[\left(a_{\alpha_{2}, k} k_{\alpha_{1}}-a_{\alpha_{1}, k} k_{\alpha_{2}}\right)-2 \sum_{l^{\prime} \in \mathbb{Z}^{4}} a_{\alpha_{1}, k-l^{\prime}} a_{\alpha_{2}, l^{\prime}} \sin \left(\frac{k \cdot \Theta l^{\prime}}{2}\right)\right] \\
& {\left[\left(a_{\alpha_{2},-k} k_{\alpha_{1}}-a_{\alpha_{1},-k} k_{\alpha_{2}}\right)-2 \sum_{l^{\prime \prime} \in \mathbb{Z}^{4}} a_{\alpha_{1},-k-l^{\prime \prime}} a_{\alpha_{2}, l^{\prime \prime}} \sin \left(\frac{k \cdot \Theta l^{\prime \prime}}{2}\right)\right] . }
\end{aligned}
$$

One checks that the term in $a^{q}$ of $\tau\left(F_{\alpha_{1} \alpha_{2}} F^{\alpha_{1} \alpha_{2}}\right)$ corresponds to the term $f\left(\mathbb{A}^{+}\right)^{q}$ given by Lemma 6.12. For $q=2$, this is

$$
-2 \sum_{l \in \mathbb{Z}^{4}, \alpha_{1}, \alpha_{2}} a_{\alpha_{1}, l} a_{\alpha_{2},-l}\left(l_{\alpha_{1}} l_{\alpha_{2}}-\delta_{\alpha_{1} \alpha_{2}}|l|^{2}\right) .
$$

For $q=3$, we compute the crossed terms

$$
i \sum_{k, k^{\prime}, l}\left(a_{\alpha_{2}, k} k_{\alpha_{1}}-a_{\alpha_{1}, k} k_{\alpha_{2}}\right) a_{k^{\prime}}^{\alpha_{1}} a_{l}^{\alpha_{2}}\left(U_{k}\left[U_{k^{\prime}}, l\right]+\left[U_{k^{\prime}}, U_{l}\right] U_{k}\right),
$$

which gives the following $a^{3}$-term in $\tau\left(F_{\alpha_{1} \alpha_{2}} F^{\alpha_{1} \alpha_{2}}\right)$ :

$$
-8 \sum_{l_{i}} a_{\alpha_{3},-l_{1}-l_{2}} a_{l_{2}}^{\alpha_{1}} a_{\alpha_{1}, l_{1}} \sin \frac{l_{1} \cdot \Theta l_{2}}{2} l_{1}^{\alpha_{3}} \text {. }
$$

For $q=4$, this is

$$
-4 \sum_{l_{i}} a_{\alpha_{1},-l_{1}-l_{2}-l_{3}} a_{\alpha_{2}, l_{3}} a_{l_{2}}^{\alpha_{1}} a_{l_{1}}^{\alpha_{2}} \sin \frac{l_{1} \cdot \Theta\left(l_{2}+l_{3}\right)}{2} \sin \frac{l_{2} \cdot \Theta l_{3}}{2},
$$

which corresponds to the term $f\left(\mathbb{A}^{+}\right)^{4}$. We finally obtain that

$$
\sum_{q=1}^{n} \frac{(-1)^{q}}{q} f\left(\mathbb{A}^{+}\right)^{q}=-\frac{c}{2} \tau\left(F_{\alpha_{1}, \alpha_{2}} F^{\alpha_{1} \alpha_{2}}\right) .
$$


Equations (6.10) and (6.11) yield the result.

Lemma 6.14. Suppose that $n=2$. Then, with the same hypothesis as in Lemma 6.11, the following holds:

(i) $f\left(\mathbb{A}^{+}\right)^{2}=f\left(\mathbb{A}^{-}\right)^{2}=0$.

(ii) Suppose that $\frac{1}{2 \pi} \Theta$ is diophantine. Then

$$
f \mathbb{A}^{+} \mathbb{A}^{-}=f \mathbb{A}^{-} \mathbb{A}^{+}=0
$$

Proof. (i) Lemma 6.11 implies that $f \mathbb{A}^{++}=\operatorname{Res}_{s=0} \sum_{l \in \mathbb{Z}^{2}}-f(s, l)$, where

$$
\begin{aligned}
f(s, l) & :=\sum_{k \in \mathbb{Z}^{2}}^{\prime} \frac{k_{\mu_{1}}(k+l)_{\mu_{2}}}{|k|^{s+2}|k+l|^{2}} \tilde{a}_{\alpha, l} \operatorname{Tr}\left(\gamma^{\alpha_{2}} \gamma^{\mu_{2}} \gamma^{\alpha_{1}} \gamma^{\mu_{1}}\right) \\
& =: f_{\mu, \alpha}(s, l) \operatorname{Tr}\left(\gamma^{\alpha_{2}} \gamma^{\mu_{2}} \gamma^{\alpha_{1}} \gamma^{\mu_{1}}\right)
\end{aligned}
$$

and $\tilde{a}_{\alpha, l}:=a_{\alpha_{1}, l} a_{\alpha_{2},-l}$. This time, since $n=2$, it is enough to apply just once (2.10) to obtain an absolutely convergent series. Indeed, with (2.10) we get

$$
f_{\mu, \alpha}(s, l)=\sum_{k \in \mathbb{Z}^{2}}^{\prime} \frac{k_{\mu_{1}}(k+l)_{\mu_{2}}}{|k|^{s+4}} \tilde{a}_{\alpha, l}-\sum_{k \in \mathbb{Z}^{2}}^{\prime} \frac{k_{\mu_{1}}(k+l)_{\mu_{2}}\left(2 k \cdot l+|l|^{2}\right)}{|k|^{s+4}|k+l|^{2}} \tilde{a}_{\alpha, l} .
$$

The function $r(s, l):=\sum_{k \in \mathbb{Z}^{2}}^{\prime} \frac{k_{\mu_{1}}(k+l) \mu_{2}\left(2 k \cdot l+|l|^{2}\right)}{|k|^{s+4}|k+l|^{2}} \tilde{a}_{\alpha, l}$ is a linear combination of functions of the type $H(s, l)$ satisfying the hypothesis of Corollary 2.14. As a consequence, $r(s, l)$ satisfies (H1) and

$$
f_{\mu, \alpha}(s, l) \sim \sum_{k \in \mathbb{Z}^{2}}^{\prime} \frac{k_{\mu_{1}}(k+l)_{\mu_{2}}}{|k|^{s+4}} \tilde{a}_{\alpha, l} \sim \sum_{k \in \mathbb{Z}^{2}}^{\prime} \frac{k_{\mu_{1}} k_{\mu_{2}}}{|k|^{s+4}} \tilde{a}_{\alpha, l} .
$$

Note that the function $(s, l) \mapsto h_{\mu, \alpha}(s, l):=\sum_{k \in \mathbb{Z}^{2}}^{\prime} \frac{k_{\mu_{1}} k_{\mu_{2}}}{|k|^{s+4}} \tilde{a}_{\alpha, l}$ satisfies (H2). Thus, Lemma 2.15 yields that

$$
\operatorname{Res}_{s=0} f(s, l)=\sum_{l \in \mathbb{Z}^{2}} \operatorname{Res}_{s=0} h_{\mu, \alpha}(s, l) \operatorname{Tr}\left(\gamma^{\alpha_{2}} \gamma^{\mu_{2}} \gamma^{\alpha_{1}} \gamma^{\mu_{1}}\right) .
$$

By Proposition 2.17, we get $\operatorname{Res}_{s=0} h_{\mu, \alpha}(s, l)=\delta_{\mu_{1} \mu_{2}} \pi \tilde{a}_{\alpha, l}$. Therefore,

$$
f \mathbb{A}^{++}=-\pi \sum_{l \in \mathbb{Z}^{2}} \tilde{a}_{\alpha, l} \operatorname{Tr}\left(\gamma^{\alpha_{2}} \gamma^{\mu} \gamma^{\alpha_{1}} \gamma_{\mu}\right)=0
$$


according to (6.4).

(ii) By Lemma 6.11, we obtain that

$$
f_{\mathbb{A}^{-+}}=\operatorname{Res}_{s=0} \sum_{l \in \mathbb{Z}^{2}} \lambda_{\sigma} f_{\alpha, \mu}(s, l) \operatorname{Tr}\left(\gamma^{\alpha_{2}} \gamma^{\mu_{2}} \gamma^{\alpha_{1}} \gamma^{\mu_{1}}\right),
$$

where $\lambda_{\sigma}=-(-i)^{2}=1$,

$$
f_{\alpha, \mu}(s, l):=\sum_{k \in \mathbb{Z}^{2}}^{\prime} \frac{k_{\mu_{1}}(k+l)_{\mu_{2}}}{|k|^{s+2}|k+l|^{2}} e^{i \eta k \cdot \Theta l} \tilde{a}_{\alpha, l}
$$

and $\eta:=\frac{1}{2}\left(\sigma_{1}-\sigma_{2}\right)=-1$. As in the proof of (i), since the presence of the phase does not change the fact that $r(s, l)$ satisfies $(\mathrm{H} 1)$, we get

$$
f_{\alpha, \mu}(s, l) \sim \sum_{k \in \mathbb{Z}^{2}}^{\prime} \frac{k_{\mu_{1}}(k+l)_{\mu_{2}}}{|k|^{s+4}} e^{i \eta k \cdot \Theta l} \tilde{a}_{\alpha, l}:=g_{\alpha, \mu}(s, l) .
$$

Since $\frac{1}{2 \pi} \Theta$ is diophantine, the functions $s \mapsto \sum_{l \in \mathbb{Z}^{2} \backslash\{0\}} g_{\alpha, \mu}(s, l)$ are holomorphic at $s=0$ by Theorem 2.63 . As a consequence,

$$
\begin{aligned}
f \mathbb{A}^{-+} & =\operatorname{Res}_{s=0} g_{\alpha, \mu}(s, 0) \operatorname{Tr}\left(\gamma^{\alpha_{2}} \gamma^{\mu_{2}} \gamma^{\alpha_{1}} \gamma^{\mu_{1}}\right) \\
& =\operatorname{Res}_{s=0} \sum_{k \in \mathbb{Z}^{2}}^{\prime} \frac{k_{\mu_{1}} k_{\mu_{2}}}{|k|^{s+4}} \tilde{a}_{\alpha, 0} \operatorname{Tr}\left(\gamma^{\alpha_{2}} \gamma^{\mu_{2}} \gamma^{\alpha_{1}} \gamma^{\mu_{1}}\right) .
\end{aligned}
$$

Recall from Proposition 2.1 that $\operatorname{Res}_{s=0} \sum_{k \in \mathbb{Z}^{2}}^{\prime} \frac{k_{i} k_{j}}{|k|^{s+4}}=\delta_{i j} \pi$. Thus, again with (6.4),

$$
f \mathbb{A}^{-+}=\tilde{a}_{\alpha, 0} \pi \operatorname{Tr}\left(\gamma^{\alpha_{2}} \gamma^{\mu} \gamma^{\alpha_{1}} \gamma_{\mu}\right)=0 .
$$

Lemma 6.15. Suppose that $n=2$ and $\frac{1}{2 \pi} \Theta$ is diophantine. Then for any selfadjoint 1-form $A$,

$$
\zeta_{D_{A}}(0)-\zeta_{D}(0)=0
$$

Proof. As in Lemma 6.13, we use (4.5) and Lemma 6.6 so the result follows from Lemma 6.14.

\subsubsection{Odd dimensional case}

Lemma 6.16. Suppose that $n$ is odd and $\frac{1}{2 \pi} \Theta$ is diophantine. Then for any selfadjoint 1-form $A$ and $\sigma \in\{-,+\}^{q}$ with $2 \leq q \leq n$,

$$
f \mathbb{A}^{\sigma}=0
$$


Proof. Since $\mathbb{A}^{\sigma} \in \Psi_{1}(\mathcal{A})$, Lemma 5.11 with $k=n$ gives the result.

Corollary 6.17. Let the same hypothesis be satisfied as in Lemma 6.16. Then for any selfadjoint 1-form $A$ we have $\zeta_{D_{A}}(0)-\zeta_{D}(0)=0$.

Proof. As in Lemma 6.13, we use (4.5) and Lemma 6.6, so the result follows from Lemma 6.16.

\subsection{Proof of the main result}

Proof of Theorem 6.1. (i) From (1.5) and Proposition 5.5 we get

$$
\mathcal{S}\left(\mathcal{D}_{A}, \Phi, \Lambda\right)=4 \pi \Phi_{2} \Lambda^{2}+\Phi(0) \zeta_{D_{A}}(0)+\mathcal{O}\left(\Lambda^{-2}\right),
$$

where $\Phi_{2}=\frac{1}{2} \int_{0}^{\infty} \Phi(t) d t$. By Lemma 6.15, $\zeta_{D_{A}}(0)-\zeta_{D}(0)=0$ and from Proposition $5.4, \zeta_{D}(0)=0$, so the result follows.

(ii) Similarly, $\mathcal{S}\left(\mathcal{D}_{A}, \Phi, \Lambda\right)=8 \pi^{2} \Phi_{4} \Lambda^{4}+\Phi(0) \zeta_{D_{A}}(0)+\mathcal{O}\left(\Lambda^{-2}\right)$ with $\Phi_{4}=$ $\frac{1}{2} \int_{0}^{\infty} \Phi(t) t d t$. Lemma 6.13 implies that $\zeta_{D_{A}}(0)-\zeta_{D}(0)=-c \tau\left(F_{\mu \nu} F^{\mu \nu}\right)$, and by Proposition 5.4 we obtain that $\zeta_{D_{A}}(0)=-c \tau\left(F_{\mu \nu} F^{\mu \nu}\right)$ and hence the result.

(iii) This is a direct consequence of (1.5), Propositions 5.4 and 5.5, and Corollary 6.17 .

\section{A. Appendix}

A.1. Proof of Lemma 3.3. (i) We have $|D| T|D|^{-1}=T+\delta(T)|D|^{-1}$ and $|D|^{-1} T|D|=T-|D|^{-1} \delta(T)$. By recurrence, $|D|^{k} T|D|^{-k}=\sum_{q=0}^{k}\left(\begin{array}{c}q \\ k\end{array}\right) \delta^{q}(T)|D|^{-q}$ for any $k \in \mathbb{N}$ and so $|D|^{-k} T|D|^{k}=\sum_{q=0}^{k}(-1)^{q}\left(\begin{array}{c}q \\ k\end{array}\right)|D|^{-q} \delta^{q}(T)$.

As a consequence, since $T,|D|^{-q}$ and $\delta^{q}(T)$ are in $O P^{0}$ for any $q \in \mathbb{N}$, for any $k \in$ $\mathbb{Z},|D|^{k} T|D|^{-k} \in O P^{0}$. Let us fix $p \in \mathbb{N}_{0}$ and define $F_{p}(s):=\delta^{p}\left(|D|^{s} T|D|^{-s}\right)$ for $s \in \mathbb{C}$. Since for $k \in \mathbb{Z}, F_{p}(k)$ is bounded, a complex interpolation proves that $F_{p}(s)$ is bounded, which gives $|D|^{s} T|D|^{-s} \in O P^{0}$.

(ii) Let $T \in O P^{\alpha}$ and $T^{\prime} \in O P^{\beta}$. Thus, $T|D|^{-\alpha}, T^{\prime}|D|^{-\beta}$ are in $O P^{0}$. By (i) we get $|D|^{\beta} T|D|^{-\alpha}|D|^{-\beta} \in O P^{0}$, so $T^{\prime}|D|^{-\beta}|D|^{\beta} T|D|^{-\beta-\alpha} \in O P^{0}$. Thus, $T^{\prime} T|D|^{-(\alpha+\beta)} \in O P^{0}$.

(iii) For $T \in O P^{\alpha},|D|^{\alpha-\beta}$ and contained in $T|D|^{-\alpha}$ are in $O P^{0}$ and therefore $T|D|^{-\beta}=T|D|^{-\alpha}|D|^{\alpha-\beta} \in O P^{0}$.

(iv) follows from $\delta\left(O P^{0}\right) \subseteq O P^{0}$.

(v) Since $\nabla(T)=\delta(T)|D|+|D| \delta(T)-\left[P_{0}, T\right]$, the result follows from (ii), (iv) and the fact that $P_{0}$ is in $O P^{-\infty}$. 
A.2. Proof of Lemma 3.6. The non-trivial part of the proof is the stability under the product of operators. Let $T, T^{\prime} \in \Psi(\mathcal{A})$. There are $d, d^{\prime} \in \mathbb{Z}$ such that for any $N \in \mathbb{N}, N>|d|+\left|d^{\prime}\right|$, there exist $P, P^{\prime}$ in $\mathcal{D}(\mathcal{A}), p, p^{\prime} \in \mathbb{N}_{0}, R \in O P^{-N-d^{\prime}}$, $R^{\prime} \in O P^{-N-d}$ such that $T=P D^{-2 p}+R, T^{\prime}=P^{\prime} D^{-2 p^{\prime}}+R^{\prime}, P D^{-2 p} \in O P^{d}$ and $P^{\prime} D^{-2 p^{\prime}} \in O P^{d^{\prime}}$.

Thus, $T T^{\prime}=P D^{-2 p} P^{\prime} D^{-2 p^{\prime}}+R P^{\prime} D^{-2 p^{\prime}}+P D^{-2 p} R^{\prime}+R R^{\prime}$.

We also have $R P^{\prime} D^{-2 p^{\prime}} \in O P^{-N-d^{\prime}+d^{\prime}}=O P^{-N}$ and, similarly, $P D^{-2 p} R^{\prime} \in$ $O P^{-N}$. Since $R R^{\prime} \in O P^{-2 N}$, it follows that

$$
T T^{\prime} \sim P D^{-2 p} P^{\prime} D^{-2 p^{\prime}} \bmod O P^{-N}
$$

If $p=0$, then $T T^{\prime} \sim Q D^{-2 p^{\prime}} \bmod O P^{-N}$, where $Q=P P^{\prime} \in \mathcal{D}(\mathcal{A})$ and $Q D^{-2 p^{\prime}} \in O P^{d+d^{\prime}}$. Suppose that $p \neq 0$. By recurrence one shows that $D^{-2} P^{\prime} \sim \sum_{k=0}^{q}(-1)^{k} \nabla^{k}\left(P^{\prime}\right) D^{-2 k-2}+(-1)^{q+1} D^{-2} \nabla^{q+1}\left(P^{\prime}\right) D^{-2 q-2} \bmod O P^{-\infty}$.

for any $q \in \mathbb{N}_{0}$. By Lemma $3.3(\mathrm{v})$, the remainder is in $O P^{d^{\prime}+2 p^{\prime}-q-3}$ since $P^{\prime} \in$ $O P^{d^{\prime}+2 p^{\prime}}$. Another recurrence gives

$$
D^{-2 p} P^{\prime} \sim \sum_{k_{1}, \ldots, k_{p}=0}^{q}(-1)^{|k|_{1}} \nabla^{|k|_{1}}\left(P^{\prime}\right) D^{-2|k|_{1}-2 p} \bmod O P^{d^{\prime}+2 p^{\prime}-q-1-2 p} .
$$

for any $q \in \mathbb{N}_{0}$. Thus, with $q_{N}=N+d+d^{\prime}-1$,

$$
T T^{\prime} \sim \sum_{k_{1}, \ldots, k_{p}=0}^{q_{N}}(-1)^{|k|_{1}} P \nabla^{|k|_{1}}\left(P^{\prime}\right) D^{-2|k|_{1}-2\left(p+p^{\prime}\right)} \bmod O P^{-N} .
$$

The last sum can be written $Q_{N} D^{-2 r_{N}}$ where $r_{N}:=p q_{N}+\left(p+p^{\prime}\right)$. Since $Q_{N} \in \mathcal{D}(\mathcal{A})$ and $Q_{N} D^{-2 r_{N}} \in O P^{d+d^{\prime}}$, the result follows.

A.3. Proof of Proposition 3.11. Let $P \in O P^{k_{1}}$ and $Q \in O P^{k_{2}} \in \Psi(\mathcal{A})$. With $\left[Q,|D|^{-s}\right]=\left(Q-\sigma_{-s}(Q)\right)|D|^{-s}$ and $Q-\sigma_{-s}(Q) \sim-\sum_{r=1}^{N} g(-s, r) \varepsilon^{r}(Q) \bmod$ $O P^{-N-1+k_{2}}$, we get

$$
P\left[Q,|D|^{-s}\right] \sim-\sum_{r=1}^{N} g(-s, r) P \varepsilon^{r}(Q)|D|^{-s} \bmod O P^{-N-1+k_{1}+k_{2}-\operatorname{Re}(s)},
$$

which gives, if we choose $N=n+k_{1}+k_{2}$,

$$
\operatorname{Res}_{s=0} \operatorname{Tr}\left(P\left[Q,|D|^{-s}\right]\right)=-\sum_{r=1}^{n+k_{1}+k_{2}} \operatorname{Res}_{s=0} g(-s, r) \operatorname{Tr}\left(P \varepsilon^{r}(Q)|D|^{-s}\right) .
$$


By hypothesis $s \mapsto \operatorname{Tr}\left(P \varepsilon^{r}(Q)|D|^{-s}\right)$ has only simple poles. Thus, because $s=0$ is a zero of the analytic function $s \mapsto g(-s, r)$ for any $r \geq 1$, it follows that $\operatorname{Res}_{s=0} g(-s, r) \operatorname{Tr}\left(P \varepsilon^{r}(Q)|D|^{-s}\right)=0$, which implies that $\operatorname{Res}_{s=0} \operatorname{Tr}\left(P\left[Q,|D|^{-s}\right]\right)=0$ and hence

$$
f P Q=\operatorname{Res}_{s=0} \operatorname{Tr}\left(P|D|^{-s} Q\right) .
$$

When $s \in \mathbb{C}$ with $\operatorname{Re}(s)>2 \max \left(k_{1}+n+1, k_{2}\right)$, the operator $P|D|^{-s / 2}$ is traceclass, while $|D|^{-s / 2} Q$ is bounded, so $\operatorname{Tr}\left(P|D|^{-s} Q\right)=\operatorname{Tr}\left(|D|^{-s / 2} Q P|D|^{-s / 2}\right)=$ $\operatorname{Tr}\left(\sigma_{-s / 2}(Q P)|D|^{-s}\right)$. Thus, using (3.1) again, we obtain that

$$
\operatorname{Res}_{s=0} \operatorname{Tr}\left(P|D|^{-s} Q\right)=f Q P+\sum_{r=1}^{n+k_{1}+k_{2}} \operatorname{Res}_{s=0} g(-s / 2, r) \operatorname{Tr}\left(\varepsilon^{r}(Q P)|D|^{-s}\right) .
$$

As before, for any $r \geq 1, \underset{s=0}{\operatorname{Res}} g(-s / 2, r) \operatorname{Tr}\left(\varepsilon^{r}(Q P)|D|^{-s}\right)=0$ since $g(0, r)=0$ and the spectral triple is simple. Finally,

$$
\operatorname{Res}_{s=0} \operatorname{Tr}\left(P|D|^{-s} Q\right)=f Q P .
$$

Acknowledgments. We thank Pierre Duclos, Emilio Elizalde, Victor Gayral, Thomas Krajewski, Sylvie Paycha, Joe Varilly, Dmitri Vassilevich and Antony Wassermann for helpful discussions as well as Stéphane Louboutin for his help with Proposition 2.17.

A. Sitarz would like to thank the CPT-Marseilles for its hospitality and the Université de Provence for its financial support and acknowledge the support of Alexander von Humboldt Foundation through the Humboldt Fellowship.

\section{References}

[1] A. L. Carey, J. Phillips, A. Rennie, and F. A. Sukochev, The local index formula in semifinite Von Neumann algebras I: Spectral flow. Adv. Math. 202 (2006), 451-516. Zbl 1118.46060 MR 2222358

[2] L. Carminati, B. Iochum and T. Schücker, Noncommutative Yang-Mills and noncommutative relativity: a bridge over troubled water. Eur. Phys. J. C Part. Fields 8 (1999), 697-709. MR 1738959

[3] A. H. Chamseddine and A. Connes, The spectral action principle. Comm. Math. Phys. 186 (1997), 731-750. Zbl 0894.58007 MR 1463819

[4] A. H. Chamseddine, A. Connes and M. Marcolli, Gravity and the standard model with neutrino mixing. Preprint 2006. arXiv:hep-th/0610241.

[5] A. Connes, C* algèbres et géométrie différentielle. C. R. Acad. Sci. Paris Sér. A 290 (1980), 599-604; translated as ' $C^{*}$ algebras and differential geometry'. arXiv:hep-th/0101093. Zbl 0433.46057MR 0572645 
[6] A. Connes, Non-commutative differential geometry. Inst. Hautes Études Sci. Publ. Math. 62 (1985), 41-144. Zbl 0592.46056 MR 823176

[7] A. Connes, Noncommutative geometry. Academic Press, San Diego 1994. Zbl 0818.46076 MR 1303779

[8] A. Connes, Geometry from the spectral point of view. Lett. Math. Phys. 34 (1995), 203-238. Zbl 1042.46515 MR 1345552

[9] A. Connes, Noncommutative geometry and reality. J. Math. Phys. 36 (1995), 6194-6231. Zbl 0871.58008 MR 1355905

[10] A. Connes, Cours au Collège de France, January 2001.

[11] A. Connes and A. H. Chamseddine, Inner fluctuations of the spectral action. J. Geom. Phys. 57 (2006), 1-21. Zbl 1105.58004 MR 2265456

[12] A. Connes and G. Landi, Noncommutative manifolds, the instanton algebra and isospectral deformations. Comm. Math. Phys. 221 (2001), 141-159. Zbl 0997.81045 MR 1846904

[13] A. Connes and H. Moscovici, The local index formula in noncommutative geometry. Geom. Funct. Anal. 5 (1995), 174-243. Zbl 0960.46048 MR 1334867

[14] A. Edery, Multidimensional cut-off technique, odd-dimensional Epstein zeta functions and Casimir energy of massless scalar fields. J. Phys. A 39 (2006), 685-712. Zbl 1087.81040 MR 2207782

[15] E. Elizalde, S. D. Odintsov, A. Romeo, A. A. Bytsenko and S. Zerbini, Zeta regularization techniques with applications. World Scientific, Singapore 1994. Zbl 1050.81500 MR 1346490

[16] R. Estrada, J. M. Gracia-Bondía, and J. C. Várilly, On summability of distributions and spectral geometry. Comm. Math. Phys. 191 (1998), 219-248. Zbl 0897.60040 MR 1603757

[17] V. Gayral, Heat-kernel approach to UV/IR mixing on isospectral deformation manifolds. Ann. Henri Poincaré 6 (2005), 991-1023. Zbl 1082.58006 MR 2189376

[18] V. Gayral and B. Iochum, The spectral action for Moyal phase. J. Math. Phys. 46, no. 4 (2005), 043503. Zbl 1067.58020 MR 2131255

[19] V. Gayral, B. Iochum, and J. C. Várilly, Dixmier traces on noncompact isospectral deformations. J. Funct. Anal. 237 (2006), 507-539. Zbl 1106.46054 MR 2230348

[20] V. Gayral, B. Iochum, and D. V. Vassilevich, Heat kernel and number theory on NC-torus. Comm. Math. Phys. 273 (2007), 415-443. Zbl 05199479 MR 2318313

[21] P. B. Gilkey, Asymptotic formulae in spectral geometry. Stud. Adv. Math., Chapman \& Hall/CRC, Boca Raton, FL, 2004. Zbl 1080.58023 MR 2040963

[22] A. de Goursac, J.-C. Wallet and R. Wulkenhaar, Noncommutative induced gauge theory. Eur. Phys. J. C Part. Fields 51 (2007), 977-987. MR 2336186

[23] J. M. Gracia-Bondía, J. C. Várilly, and H. Figueroa, Elements of noncommutative geometry. Birkhäuser Adv. Texts, Birkhäuser, Boston 2001. Zbl 0958.46039 MR 1789831

[24] V. W. Guillemin, S. Sternberg, and J. Weitsman, The Ehrhart function for symbols. Preprint 2006. arXiv:math.CO/0601714 
[25] G. H. Hardy and E. M. Wright, An introduction to the theory of numbers. 5th ed., The Clarendon Press, Oxford 1979. Zbl 0423.10001 MR 0568909

[26] N. Higson, The local index formula in noncommutative geometry. In Contemporary developments in algebraic K-theory, ICTP Lecture Notes 15, Abdus Salam Int. Cent. Theoret. Phys., Trieste 2003, 447-536. Zbl 02143530 MR 2175637

[27] T. Kato, Perturbation theory for linear operators. 2nd ed., Grundlehren Math. Wiss. 132, Springer-Verlag, Berlin 1976. Zbl 0342.47009 MR 0407617

[28] M. Knecht and T. Schücker, Spectral action and big desert. Phys. Lett. B 640 (2006), 272-277. MR 2275783

[29] R. Nest, E. Vogt, and W. Werner, Spectral action and the Connes-Chamsedinne model. In Noncommutative geometry and the standard model of elementary particle physics (Hesselberg, 1999), Lecture Notes in Phys. 596, Springer, Berlin 2002, 109-132. Zbl 1042.81106 MR 1998532

[30] M. A. Rieffel, $C^{*}$-algebras associated with irrational rotations. Pacific J. Math. 93 (1981), 415-429. Zbl 0499.46039 MR 623572

[31] M. A. Rieffel, Deformation quantization for actions of $\mathbb{R}^{d}$, Mem. Amer. Soc. 106 (1993), no. 506. Zbl 0798.46053 MR 1184061

[32] L. Schwartz, Méthodes mathématiques pour les sciences physiques. Enseignement des Sciences, Hermann, Paris 1961. Zbl 0101.41301 MR 0143360

[33] B. Simon, Trace ideals and their applications. London Math. Soc. Lecture Note Ser. 35, Cambridge University Press, Cambridge 1979. Zbl 0423.47001 MR 0541149

[34] A. Strelchenko, Heat kernel of nonminimal gauge field kinetic operators on Moyal plane. Internat. J. Modern Phys. A 22 (2007), 181-202. Zbl 1110.81156 MR 2293683

[35] W. van Suijlekom, Private communication.

[36] D. V. Vassilevich, Noncommutative heat kernel. Lett. Math. Phys. 67 (2004), 185-194. Zbl 1053.81083 MR 2078160

[37] D. V. Vassilevich, Heat kernel, effective action and anomalies in noncommutative theories. J. High Energy Phys. 08 (2005), 085. MR 2166111

[38] D. V. Vassilevich, Induced Chern-Simons action on noncommutative torus. Mod. Phys. Lett. A 22 (2007), 1255-1263. Zbl 05175420 MR 2335197

Received April 16, 2007

D. Essouabri, Université de Caen (Campus II), Laboratoire de Mathématiques Nicolas Oresme (CNRS UMR 6139), B.P. 5186, 14032 Caen, France

E-mail: essoua@math.unicaen.fr

B. Iochum , C. Levy, UMR 6207 Unité Mixte de Recherche du CNRS et des Universités Aix-Marseille I, Aix-Marseille II et de l'Université du Sud Toulon-Var, Laboratoire affilié à la FRUMAM - FR 2291 and Université de Provence, France

E-mail: iochum@cpt.univ-mrs.fr; levy@cpt.univ-mrs.fr

A. Sitarz, Institute of Physics, Jagiellonian University, Reymonta 4, 30-059 Kraków, Poland E-mail: sitarz@if.uj.edu.pl 